Portland State University

PDXScholar

1-23-1992

\title{
Developing Image Processing Tools in X Window System
}

\author{
Xin Zhang \\ Portland State University
}

Follow this and additional works at: https://pdxscholar.library.pdx.edu/open_access_etds

Part of the Computer Engineering Commons Let us know how access to this document benefits you.

\section{Recommended Citation}

Zhang, Xin, "Developing Image Processing Tools in X Window System" (1992). Dissertations and Theses. Paper 4571.

https://doi.org/10.15760/etd.6455

This Thesis is brought to you for free and open access. It has been accepted for inclusion in Dissertations and Theses by an authorized administrator of PDXScholar. Please contact us if we can make this document more accessible: pdxscholar@pdx.edu. 
AN ABSTRACT OF THE THESIS OF Xin zhang for the Master of Science in Electrical and computer Engineering presented January 23,1992 .

Title: Developing Image Processing Tools in X Window system. APPROVED BY THE MEMBERS OF THE THESIS COMMITTEE:

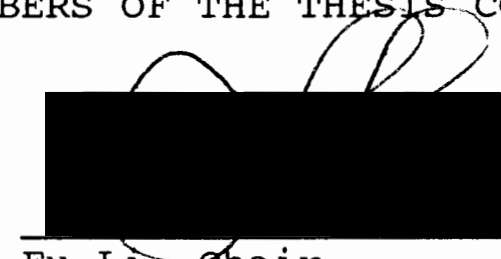

Fu Li, ehair

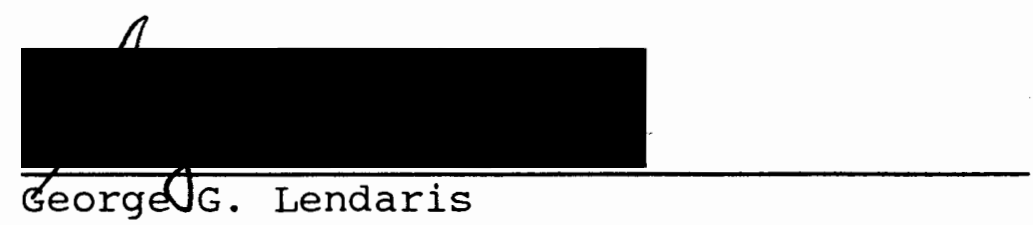

Faryar Etesami

The $\mathrm{x}$ window system is an industry-standard software system which facilitates programmers to develop portable graphical user interfaces. This thesis describes an image processing tool developed under the $\mathrm{x}$ Window system. A multiwindow image display software with image editing and improvement functions is developed. The software has four modules: environment generation, image creation, image editing and image improvement. 
The environment generation module creates working windows, scrollbars, dialogbox and pulldown menu buttons, and tracks mouse cursor positions. The working windows are three adjacent windows allowing display of three different images simultaneously. The dialogbox provides an interface between the user and the program, such as input new image and parameters. The pulldown menus offer various selections of image processing functions. A pair of scrollbars are also added to allow users to move the magnified image up, down, left and right so that the full picture can be viewed.

The image creation module can build colormap, load image data file, convert pixel values to screen color values, create image, and display image on the screen of a workstation. The program can read either sun.rasterfile format or a plain data file. If a plain data file is detected, the software can transform it to sun. rasterfile by adding an appropriate header and a colormap. There are two kinds file saving features: screen capture and store. The screen capture allows the user to save that part of image displayed on the screen. The store function saves the entire image which may be either the screen size or bigger size image (e.g. merged or zoomed image).

The image editing module is equipped with the functions for zooming (in or out), merging, rotating and restoring images. The merge function constructs a new image based on the user selected or input image from the dialogbox. The rotation center of an image can be any point selected by user's 
clicking mouse button, and the rotation angle is supplied by user in the dialogbox.

The image improvement module consists of some image point transformation and image enhancement functions.

This software package is easier to use than existing tools for image processing. It has been used in the image processing projects and related educational purposes. The software provides a framework and can be easily extended to other potential applications. 
DEVELOPING IMAGE PROCESSING TOOLS IN X WINDOW SYSTEM

by

XIN ZHANG

A thesis submitted in partial fulfillment of the requirements for the degree of

\author{
MASTER OF SCIENCE \\ in \\ ELECTRICAL AND COMPUTER ENGINEERING
}

Portland State University

1992 
TO THE OFFICE OF GRADUATE STUDIES:

The members of the committee approve the thesis of Xin Zhang presented January 23, 1992 .

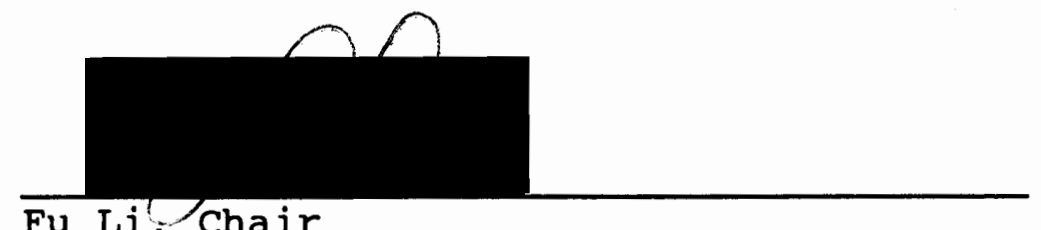

Fu Li, Chair

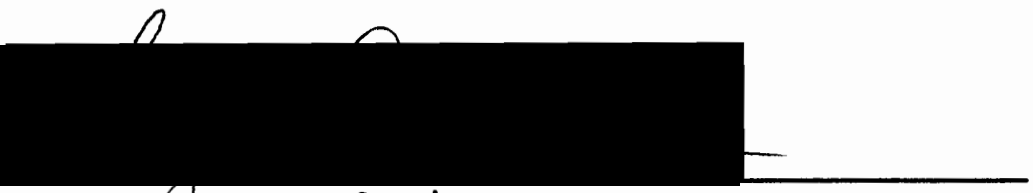
George/G. Lendaris

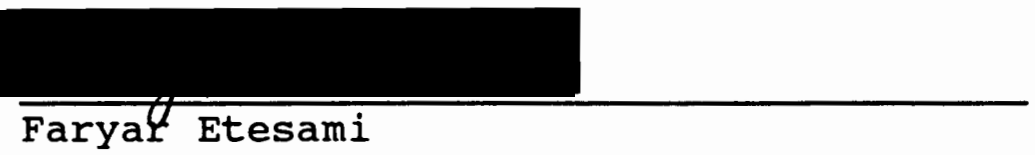

\section{APPROVED :}
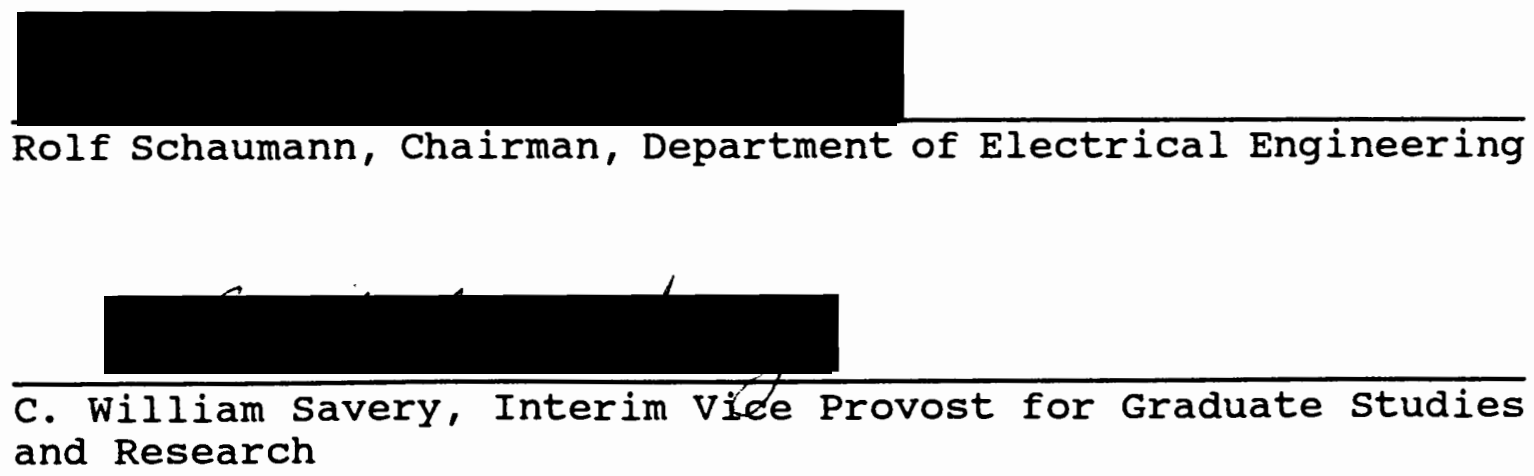


\section{ACKNOWLEDGEMENTS}

I would like to express my sincere and profound gratitude to all the members of my Graduate thesis committee for their guidance and support that has helped me in the successful completion of the project. My advisor, Dr. Fu Li, has not only introduce me to the field of my research and guided me throughout the making of this thesis, but has also provided me with encouragement and support during the course of this project. I would like to thank Dr. George G. Lendaris and Dr. Faryar Etesami for their advises, suggestions and precious time on reviewing my thesis. I would like to specially thank Ms Shirly clark for providing all kinds of help during my entire academic years in Portland State University. Finally, I would like thank my family and all my friends for giving me spiritual support. 
TABLE OF CONTENTS

PAGE

ACKNOWLEDGEMENTS. . . . . . . . . . . . . . . iii LIST OF FIGURES . . . . . . . . . . . . . . . . . vi vi

CHAPTER

I INTRODUCTION . . . . . . . . . . . . . 1

Review of $\mathrm{x}$ window system. . . . . . 3

Structure of the window System . . . . 6

Visual Class . . . . . . . . . 8

II PROGRAMMING WITH X WINDOW SYSTEM . . . . . . 11

Data Structure . . . . . . . . 11

x Color Strategy .......... 13

X Event-driven Programming Model . . . 16

An Algorithm of Image Display Program

with xlib. . . . . . . . . . 18

III PROGRAMMING WITH XT INTRINSIC

AND X TOOLKITS. . . . . . . . . . . 25

Introduction to x Toolkits . . . . . 25

Basic Steps of $\mathrm{Xt}$ Intrinsic

Programming. . . . . . . . . . 26

IV PROGRAM IMPLEMENTATION . . . . . . . . . . 29

The Working Environment Module . . . . 30

Image Creation Module. . . . . . . . 40 
Image Editing Module . . . . . . . . 42

Image Improvement Module. . . . . . 46

$\mathrm{V}$ CONCLUSION . . . . . . . . . . . . . 57

Improvement and Future Work. . . . . . 57

other system study and Comparison. . . . 59

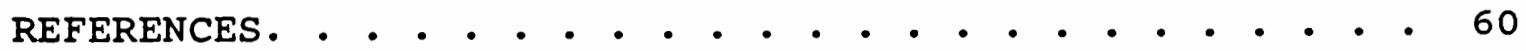

APPENDICES

A PROGRAM: XSHOW.C. . . . . . . . . . 61

B RESULTS . . . . . . . . . . . . . 106 


\section{LIST OF FIGURES}

FIGURE

PAGE

1. X network protocol . . . . . . . . . . . 4

2. Network transparency diagram . . . . . . . . 5

3. A three-level window hierarchical structure. . 7

4. Pseudocolor and Staticcolor. . . . . . . . 14

5. General structure of an event-driven program . . 16

6. An algorithm of image display program. . . . . 19

7. X Toolkits structure . . . . . . . . . 26

8. Program modules. . . . . . . . . . . . 29

9. Widget tree structure. . . . . . . . . . 30

10. Pull-down menu buttons and dialogbox widget tree . . . . . . . . . . . 34

11. The dialbox routine structure. . . . . . . 36

12. Image point transformation module. . . . . . 46

13. Directional averaging noise filter . . . . . . 54

14. Waveforms in an unsharp masking image enhancement system. . . . . . . . . 55

15. output result. . . . . . . . . . . . 107

16. Output result. . . . . . . . . . . 107 
CHAPTER I

INTRODUCTION

In recent years, a new window system has been developed and introduced by the Massachusetts Institute of Technology (MIT) known as $\mathrm{x}$ Windows. It is a software environment for engineering workstations which offers a rich, comprehensive environment and a better user graphical interface to the workstation hardware [1]. Compared with other window systems, such as Sun Tools and Sun View window systems, $\mathrm{X}$ Window system has the advantage of being network transparent, device independent and concurrent. These make $x$ window more convenient in industrial applications and in engineering program development. More detailed discussion of these is in Chapter II.

This project develops multi-window image display software with some image editing and improvement functions using the $x$ window system. The software provides a display with three adjacent windows to allow displaying three different images simultaneously. The dialogbox provides an interface between users and programs, such as new image and parameter input. Moreover, pull down menus give various selections of image processing functions (e.g. image enhancement, image noise filtering and image transformation). 
A pair of scroll bars are also added to allow users to move the magnified image picture up, down, left and right so that the full picture can be viewed. The program can read either sun.rasterfile format or a plain data file. If a plain data file is detected, the software transforms it to sun.rasterfile format by adding an appropriate header and a colormap. There are two kinds of file saving features: screen capture and store. The screen capture allows the user to save that part of image displayed on the workstation screen. The store function saves the entire image which may be the screen size or a bigger size image (e.g. merged or zoomed image).

Chapter III describes how to develop the program with Xlib utilities. An algorithm of the grey scale and color image display will also be discussed. The key point in this chapter is about how to build a colormap, create an image and display it on the workstation.

In chapter IV and V, XToolkits and widgets will be introduced. They are the higher level layers between user applications and XIib. Because $X$ does not support any particular interface style, it is said to be policy-free. The easiest way for a programmer is to deal with xtoolkits and Widgets combined with Xlib utilities rather than Xlib only. An algorithm of image display software with menu-driven features will be provided.

Chapter $\mathrm{V}$ is the conclusion of this project. It includes the other window and image processing system studies, 
comparison of them and suggested future extensions of the current work.

REVIEW OF X WINDOW SYSTEM

The foundation of $\mathrm{X}$ is the base window system which is a local $\mathrm{x}$ sever. It provides an interface to the $\mathrm{x}$ network. The $\mathrm{X}$ network protocol is designed to work either within a CPU or between cPUs. The user's interface with a workstation is the combination of the capabilities provided by the base window system, $\mathrm{X}$ Toolkits, window and session managers, and application software.

The $\mathrm{X}$ network protocol uses a programming model known as the client-server model in which the working software running in the graphics workstation is the sever performing tasks requested by client application [1]. $x$ clients are applications that use the workstation's display. The protocol, the display server, the client applications, and the Xlib library routines are collectively referred to as the $\mathrm{X}$ window System.

$\mathrm{X}$ application does not have to use the network protocol directly, but rather through a programming interface $\mathrm{xlib}$ which is a c-language subroutine package provided with $\mathrm{X}$ Window system. Although the $\mathrm{xlib}$ is very convenient, its capabilities are very basic. Some applications may access the high-level Toolkits to implement objects such as buttons, lists, and menus. These may save hundreds of lines of codes 
compared to deals with Xlib only. However, many applications, especially those techniques used by interactive graphics, will have to use XIib directly. The relations are shown in Figure 1.

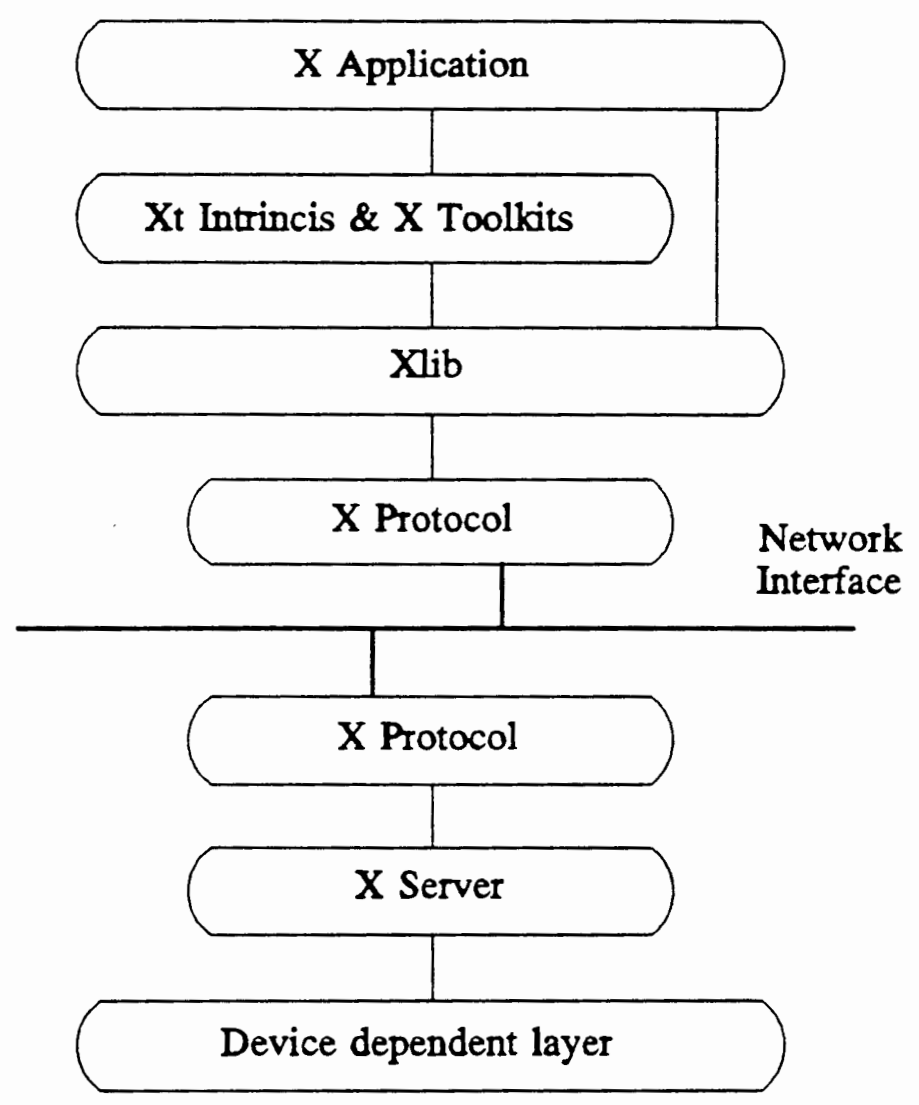

Figure 1. X network protocol.

The advantage of $\mathrm{x}$ is that its architecture hides most of the details of the device-dependent implementation of the server and the hardware. Multiple clients can be connected to a single server concurrently. A single client can also be connected to multiple servers. The network transparency, 
vendor and model independent, and concurrency are the three major characteristics of $\mathrm{x}$ window system.

\section{Network Transparency}

Network transparency means the $\mathrm{X}$ application running on one CPU can also display it's output on the screens which

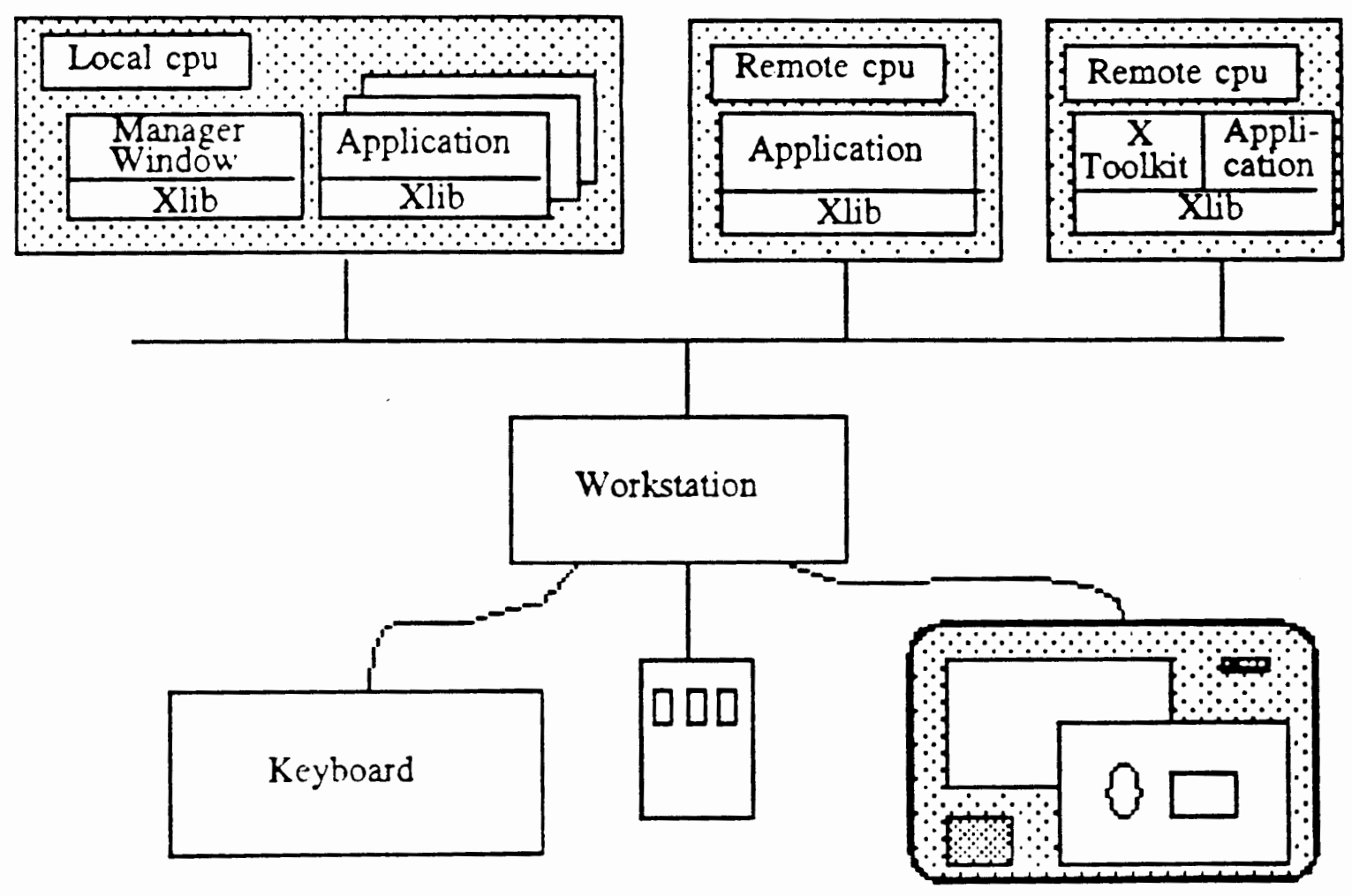

Figure 2. Network transparency diagram.

connect to the same CPU or some other CPUs. This implies that applications can run on any CPU which is the most convenient to the users.

For example, an application with a big memory requirement can run on a mainframe computer with its information displayed 
on many workstation screens (See Figure 2). To a user, an X workstation may be seen as connected to many different host CPUs at the same time.

Vendor and Model Independence

Applications operate with $\mathrm{x}$, rather than workstation's display hardware. If an application can successfully run on one workstation, it will run on any other workstations. It is not necessary to recompile or relink the application in order to execute it on other kinds of $\mathrm{X}$ workstations. This is also referred as the portability of $\mathrm{X}$ applications.

\section{Concurrency}

The $\mathrm{X}$ window system allows several applications to be active on one workstation simultaneously. This means that applications can operate either dependently or independently since the applications usually use separate or hierarchical windows to display their outputs.

\section{STRUCTURE OF THE WINDOW SYSTEM}

This section is a brief review of Windows, Window manipulation and Visual Class of $\mathrm{X}$.

\section{Window Hierarchy}

A window is a rectangular area on a workstation screen. Windows are the means by which $\mathrm{X}$ organizes many aspects of the workstation's operations, including graphical output as well as input from the workstation's mouse and keyboard [2]. 
An application can open many windows at once. The window may not be seen unless it is mapped. Unmapping a window will lose its graphic context which will have to be redrawn the when a window is mapped again.

All windows in $X$ are organized in a hierarchical structure (Figure 3 is a three-level window structure).
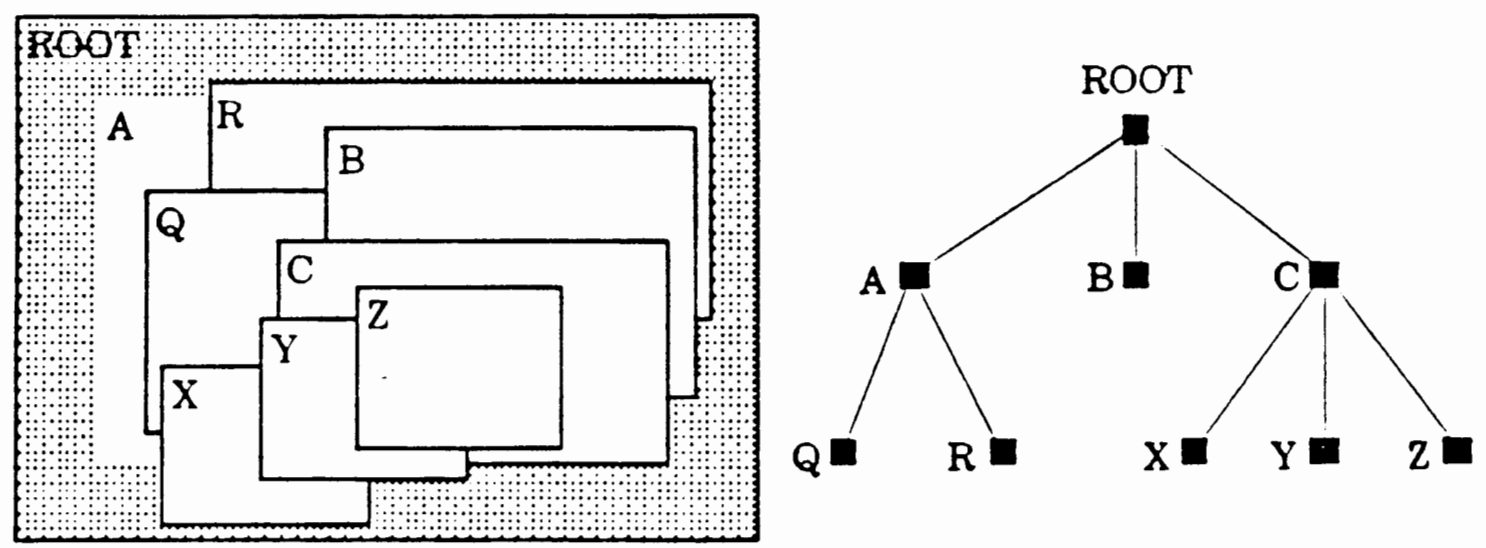

Figure 3. A three-level window hierarchical structure.

At the top of the hierarchy, is the root window which occupies the entire screen. Every window, except the root, must have a parent. The root window is always there when a user logs in to the $x$ server. The main window of an application is the child of root window.

In the window hierarchy, the visibility is determined by the stacking order. A hidden window can be seen by moving it on the top of the latest opened window. As shown in Fig. 1.3, all windows are blocked by window $\mathrm{Z}$. These windows are called obscured or partially obscured. Child windows with the same 
parent are called siblings [1]. Windows $X, Y$ and $Z$ are siblings, and all children of $C$. The windows $Q$ and $R$ are the children of window A. Windows can be raised only to the top of the same hierarchy branch. For example, window A can be not moved to the top of $\mathrm{Z}$ since they are not on the same parent-child branch. To make a window visible, the XRaisewindow and $\mathrm{X}$ Mapwindow command can be used to raise the window order in stack and map it. The short cut is to use the XmapRaised command.

All windows are managed by the window manager. It is another window application running on the workstation. The window manager works by soliciting special window-manager events from root window and from top-level application windows. What the window manager actually does with the hints that a user gives it is up to the window manager's developer. The window manager hints are stored in the workstation in the form of window's properties. You can get and set the values of each hint at any time.

\section{VISUAL CLASS}

Based on the hardware, workstations usually can be classified as either black-and-white or color. A color workstation has $2^{\text {planes }}$ colors which depends on the frame buffer memory. The number of bits in a frame buffer corresponding to one pixel is known as a plane. Each distinct pixel value serves as an index to a color map which drives the display. There are several different methods to apportion the primary 
red, green and blue to these bits. For example, if a workstation has a 24 bit frame buffer, with eight bits each dedicated to representing the actual values of red, green and blue. An 8 bit frame buffer may be divided to $(3,3,2)$ each to represent red, green and blue. These different strategies of translating pixel values into colors on a screen are called visual classes in the $\mathrm{X}$ window system. The $\mathrm{X}$ window system has $\mathrm{six}$ visual classes. Most workstations support only one of the six, while others may allow users to use several visual classes at the same time. The classification of the visual classes is as follows [2]:

\section{Pseudocolor}

On a Pseudocolor screen, pixel values index a colormap to produce independent, dynamically changeable red, green and blue values. Each pixel is treated as a single index into a single color map array.

\section{staticcolor}

This is similar to Pseudocolor, except that the contents of the color map are predefined and cannot be changed.

\section{Grayscale}

This type is also similar to Pseudocolor, except only a single primary color is available. Even a color map with red, green and blue primaries is available (the red, green and blue primaries should be equal to each other in the color map), but only one of the primaries drives the video screen. 


\section{StaticGray}

Screens with this type have a fixed, predetermined monochrome color map. The contents of staticGray can not be hanged.

\section{DirectColor}

In Directcolor visual class, the pixel value is decomposed into separate red, green and blue field. Each component is used as an index into a separate color lookup table. Directcolor requires more bits per pixel than Pseudocolor, but allows more colors and shades to be displayed simultaneously.

\section{Truecolor}

This visual type is similar to Directcolor, except that the contents of a Truecolor color map are predefined to be a linear or near-linear ramp, and cannot be changed. 
CHAPTER II

PROGRAMMING WITH X WINDOW SYSTEM

This chapter will discuss the image data structures and color strategies which are the key in the $\mathrm{X}$ programming. Also the basic steps of $\mathrm{x}$ programming, event-driven programming, will be introduced. The last section of this chapter is devoted to the topic about how to program with $\mathrm{xlib}$ and an algorithm of the gray-scale image display using Xlib.

\section{DATA STRUCTURE}

Xlib provides supports for image manipulation. As mentioned earlier, the $x$ display connection between the application and the workstation is the network link. It is hard to handle an image in the $\mathrm{x}$ environment for two reasons. The first is that an image typically contains a large amount of data, therefore Xlib has to take a substantial amount of time to convert such an image to protocol wire-format and transmit it over a display connection. The second is that different computer models often encode the bits within pixels in different orders, such as big endian or little endian types. Xlib must take these into account when transmitting images over a display connection. To reduce the effect of these difficulties, the designer of Xlib chose to represent 
images inside application programs using data structures known as XImage structures. However, the creation and delete functions, as well as the functions of operating on pixel values in XImage structures are provided by Xlib. An application can manipulate an image internally (by reading and writing pixel values) using Xlib utility functions. And, Xlib also provides requests of transferring pixels in XImage structure back and forth between workstations and applications. The standard form of XImage structure is defined as following:

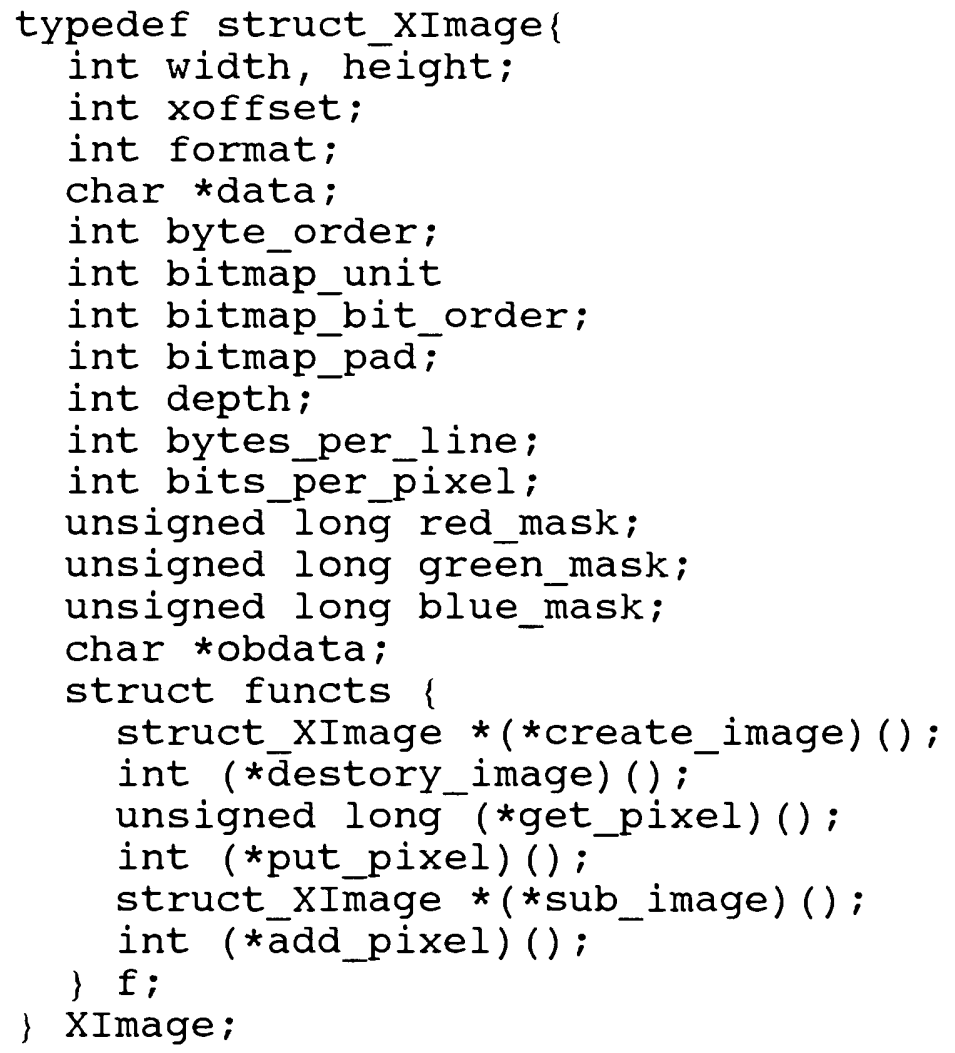

The XImage structure defines such elements as format(Bitmap, XYPixmap or ZPixmap), image width and height, 
colormask(red, green, blue). The bitmap_bit_order field determine the bit order(Lastsignificant or Mostsignificant). It describes the bit and byte ordering, and may vary depending on the type of the CPU on which the application is running. With well defined XImage data structure, one can write image manipulation code refers to it directly.

\section{COLOR STRATEGY}

Obviously, color can help in conveying more information in a more attractive way to users. $x$ uses a flexible color model that provides a common interface to many different types of display hardware. $X$ has functions which allow user applications to inquire the color capabilities of the workstation so that a suitable visual class can be used in the application.

Monitors supplied with color workstations display three primary colors, red, green and blue as well as combinations of three primary colors. Three signals drive separate electron sources inside the kinescope tube. One of these electron sources lights the myriad of red dots or lines on the surface of the tube. A second one lights the green and the third one lights the blue. The colored dots or lines on the surface of the tube are small enough that a user's eyes does not usually distinguish them. Visually, they appear to mix with color effect. The combinations of three primary colors can be used to create large quantities of distinct colors and shades. A 
set of all possible colors that a workstation screen can display is known as the screen's gamut.

A screen is composed of a large quantity of pixels. Different pixel values together determine the image shape and colors on the screen. On a black-and-white screen, each pixel is represented by a single binary digit which is a display depth of one. On a color screen, the n-bit pixels can make a depth greater than one. For example, a workstation with a color screen may have an eight-bit pixel value ranging from 0 to 255 with each pixel on screen represented by eight bits.

The $\mathrm{x}$ color model uses a colormap. It may also be called a color lookup table. A colormap is an array of color cells which provides a level of indirection between the color indexes used by the application and its display screen. An

Frame Buffer

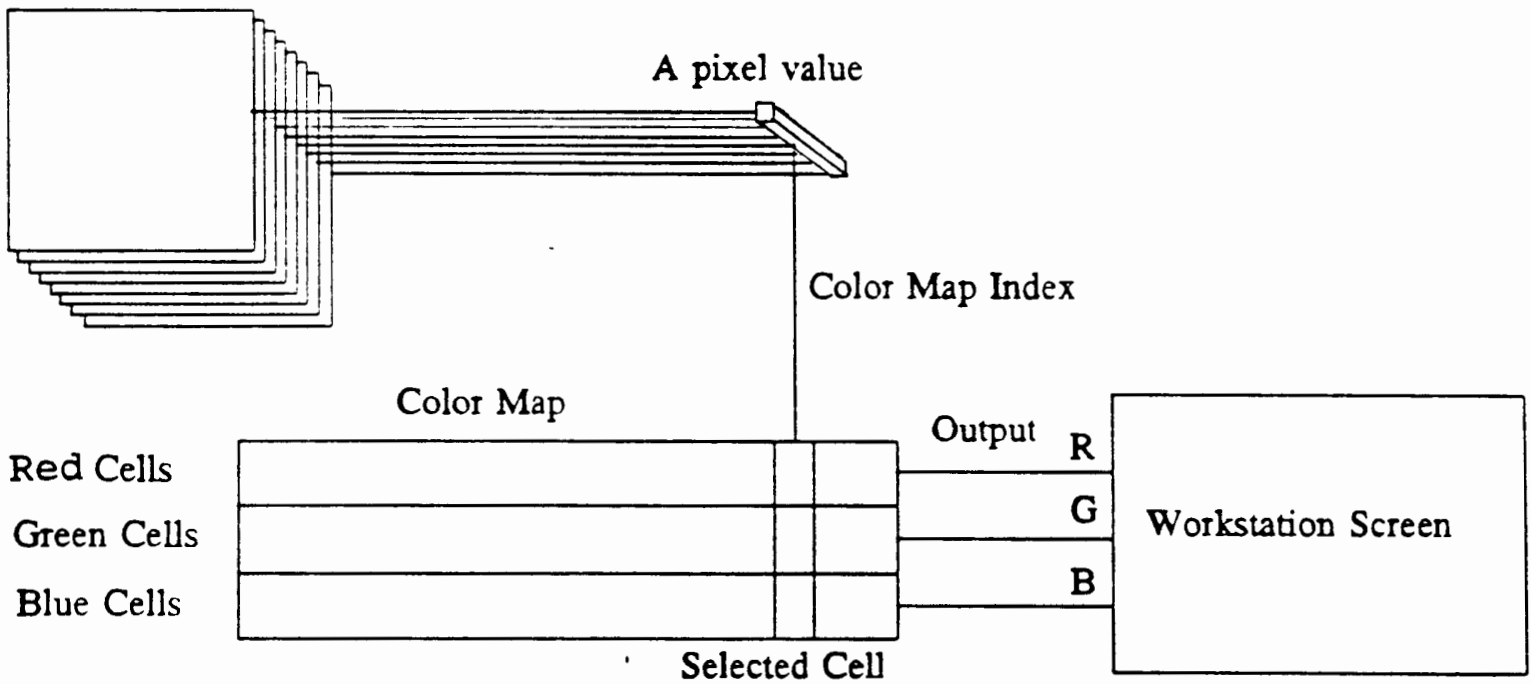

Figure 4. Pseudocolor and staticColor. 
application uses the xcolor structure to describe the contents of each color cell. Some displays may provide a hardware colormap to increase colormaping speed. Figure 4 shows a pixel value from an eight-plane display being used as an index into a cell in a colormap.

$\mathrm{X}$ Window system applications use xcolor structures to specify red-green-blue values which, in turn, manipulate colors. The definition of Xcolor structure is shown as follows:

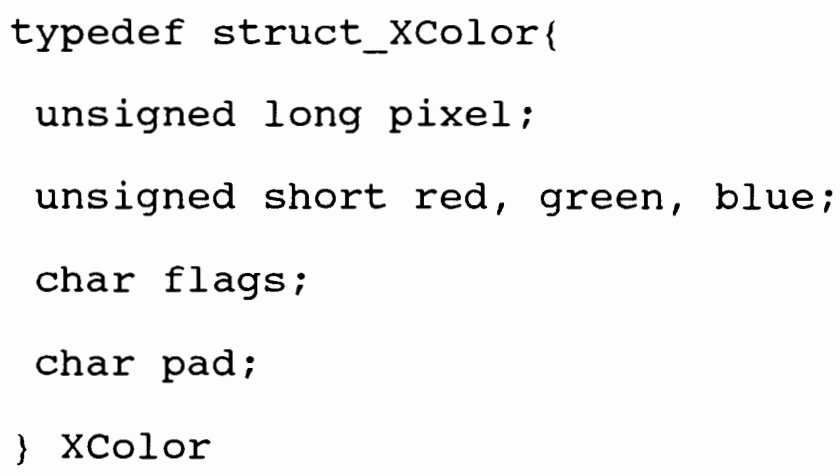

In this structure, the "red," "green," and "blue" fields represent primary color components as 16-bit numbers, valued from 0 to 65535 representing scaled intensities. Most workstations do not utilize all 16 bits of such color primary specification. They ignore all but the most significant 8 bits. This intensity allows $x$ window system applications to specify primary color component values in a way that is independent of the workstation hardware. The pixel field is used to store the pixel value associated with the red-greenblue triplet in the structure. 
X EVENT-DRIVEN PROGRAMMING MODEL

Applications designed for window system are different from traditional applications. Several modes in a window application are put together. A user can open different modes by selecting an item from a list, such as opening a file, copying a file, or printing a file. To achieve this ability, the application's developer must follow a programming model in which the application continuously checks what the user wants and performs the appropriate tasks to fulfill those requests. This programming model is known as an event-driven model because task requests coming from the mouse pointing device or from the keyboard are reported to the application as events. A typical event-driven program has three major sections [1].

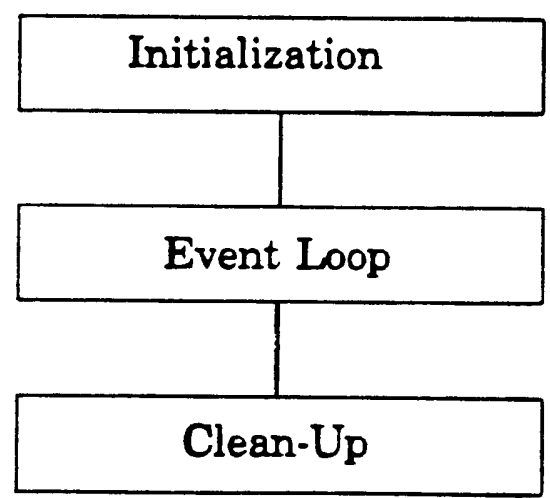

Figure 5. General structure of an event-driven program. 
The initialization section sets up the window system for user interaction. It creates a number of resources in the $\mathrm{x}$ server that the application will use for displaying outputs and receiving inputs from users. These resources can be windows, fonts, colormaps, and graphics contexts(GCs). In general, the initialization of an $\mathrm{X}$ application involves following actions:

1. Opens a connection to display.

2. Sets up fonts and colors based on user's choices.

3. Creates the top-level window of the application.

4. Informs the window manager of the window's title, size, position, and minimum width and height.

5. Creates a graphic context (GC) for the window.

6. Selects which of the input events occurring in the window will be processed by the application.

7. Makes the top-level window visible (map it).

8. Creates other windows in the hierarchy and map them. Creates more graphics contexts, if needed.

Once initialization has been done, the application is ready to handle events. In an event-driven programming model, the application does all of its work in the event loop. An event loop is often implemented as a while loop in which the program reads, interprets, and processes events.

The event loop is a crucial part of every $\mathrm{x}$ application. Since events control everything an application does, even the 
initial drawing in a window must occur only in response to a special event known as the expose event. The $\mathrm{X}$ sever maintains queues of the events, one for each client. It sends the events to its client as they occur. The Xlib then buffer the events in a queue at the client side. Xlib pops up the events, one at a time, when a client asks for an event using one of the Xlib functions.

In the last step, an X application should destroy the resources it created on the server before it exits because they consume memory in the server.

\section{AN ALGORITHM OF IMAGE DISPLAY PROGRAM WITH XLIB}

This section will introduce the algorithm of an image display program. The program uses X's base window system and Xlib functions to generate a window which is used to display a gray scale image on $\mathrm{x}$ workstation. It is difficult to use $\mathrm{Xl}$ ib dealing with $\mathrm{X}$ window manager directly and to create menu buttons, although it provides some convenient functions to build a colormap, generate an image and transfer an image to workstation screens. In the next two chapters, we will discuss how to use the higher level $x$ Toolkits combining with Xlib functions to achieve event-driven programming model result with the button and menu features.

Pixels on an $X$ screen are determined by $x$ and $y$ coordinates and their color bright level. The different bright level pixels form an image on the terminal screen. To display 
an image on the screen is actually to open a working window and to change the bright level of all the pixels according to the image data file. The model of an image display program is shown in Figure 6 below.

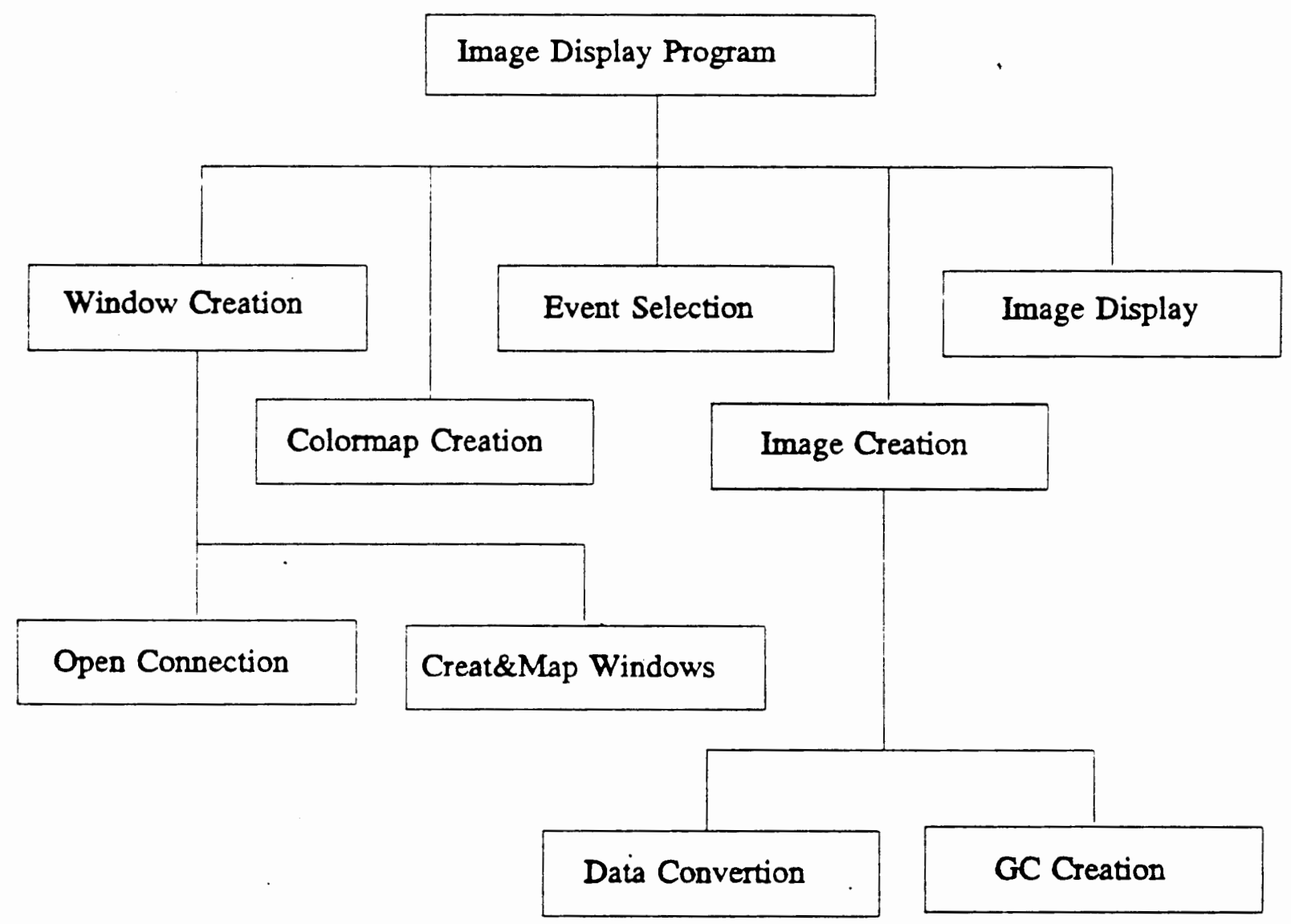

Figure 6. An algorithm of image display program.

These are the necessary procedures to display a image on a $X$ workstation which are described in detail as following.

\section{open connection}

Initialization of a $\mathrm{x}$ window system can be achieved by using the xopenDisplay. It initiates a display connection 
application through TCP or DECnet, or through some local inter-process communication protocol to the workstation, creates and files in a Display structure describing that connection, and returns a pointer to the Display structure. Create Windows:

To create a window which is a rectangular area on the screen that lets you view graphic output, we must first connect the application program to the $\mathrm{x}$ server by calling XopenDisplay. The XCreatWindow request can be used to create an unmapped application's window. This call returns the window's resource identifier.

Map Windows

After a window is generated, it may not be seen until it is mapped. As mentioned in the last chapter, the window is a drawable, many of which can be opened in a window hierarchy (depends on the hardware of workstation). In order to make them visible, the XMapRaisewindow function must be used to raise and map the windows in their hierarchy.

\section{Build Colormap}

Colormap is generated during the process of building colormap. The key to display an image on the workstation is to build the colormap so that the $\mathrm{x}$ can convert the pixel values to colors on the screen. According to the capability of the available SUN workstation with an 8 bits frame buffer, the Pseudocolor strategy is suitable for the system using this 
strategy; the value stored in the pixel is interpreted as an index to a color cell rather than the actual color. The maximum color map entry will be 256. Since the $\mathrm{X}$ color structure defines its red, green and blue are unsigned short which is 16-bit long, we can use a loop 65535/256*i(i from I to 256) to assign the red, green and blue part to build 256 colormap entry. For a grayscale image display, the value to be assigned to $(r, g, b)$ is equal. The XAlloccolor function can be employed to allocate memory and generate index value each time within the loop. Therefore, the 256 colormap cells are built in this process.

\section{Image Data Input}

At this moment the opened window is still empty, and data needs to be read in, then the images generated by graphic primitive requests can be drawn into this window. Since our image data file is either grayscale image or the single color of $(r, g, b)$ data file, they are all 1 byte long with 256 bright level. The $\mathrm{C}$ function fread can be used to read them in byte by byte and store in a character array. The values of those characters are used as indexes of a colormap. By searching the colormap, the appropriate triplet $(r, g, b)$ can be found out to fill the image data structure. The type of image format must be defined as ZPixmap. A ZPixmap format is often known as a "pixel-mode" image, and is most useful with 8 bit or 32 bit pixel values. 


\section{Generate GC}

The $\mathrm{x}$ window system provides requests to allow you to draw objects such as points, lines, rectangles and images so you don't have to draw on screen pixel-by-pixel. These objects are known as graphic primitives which is stored as a raster. When users issue object drawing requests by giving the identifiers of two $x$ resources, Drawable and GC, $X$ can figure out which pixels should be illuminated and display the picture you wanted on the screen. The drawable can be either a window or a Pixmap. The GC is known as graphic context which gives the specifies the rendering attributes to use when drawing. At least one GC must be created before the workstation display image on the screen. The XCreateGC() function can be called to create GC. Unlike windows, you can create as many GCs as you want because GC does not take much memory.

\section{Display Image}

Once an image was prepared in the application's memory, it can be drawn into a Pixmap or a window on the workstation using the XPutImage() request. Like the other graphic primitive requests, it uses a GC to set up the graphics pipeline. During this process, the important thing is that the depth of the source image must be the same as the depth of destination drawable. 
Event Selection

The event-driven $\mathrm{X}$ programming model relies on events for everything from maintaining the contents of a window to receiving input instruction from user. While using an application program, a user moves the mouse, presses and releases the mouse button, and types on the keyboard. Each of these actions generates a time-stamped event, which is delivered via an event queue to the application that wants it.

An application never receives unsolicited input events. An event is delivered only to clients who have explicitly solicited them before they occur. The selectInput request can be used to solicit events that the user wants from each window. The presence of a bit in the event_mask argument of XselectInput solicits event: delivery, and the absence disables it. Each application has a single queue of its own. The workstation usually reads events from the queue in the same order that they were delivered by using the XNextEvent request. This is a blocking call it waits until the XNextEvent call true. Hence XNextEvent request is normally used function to retrieve events from the queue and another function XEventQueued is generally used to look at the queue.

\section{clear up}

The XFreeGC, XDestroywindow and XDestoryImage requests are very important at saving memory space. Since $\mathrm{x}$ programming is the event-driven model, many resources such GC and window can be generated in a queue. But for the reason of memory 
limitation, the resources should be destroyed as long as they are not useful. When XCloseDisplay request is called, the workstation destroy all Gcs and windows automatically. 


\section{CHAPTER III}

\section{$\mathrm{X}$ PROGRAMMING WITH XT INTRINSIC AND $\mathrm{X}$ TOOLKITS}

This chapter introduces the higher level X Toolkits which include the $\mathrm{xt}$ Intrinsic and $\mathrm{x}$ widgets. The $\mathrm{x}$ Toolkits help the Xlib to manipulate the images and provide the interface between user and application. The steps of programming with $\mathrm{X}$ Toolkits will be discussed in section two.

\section{INTRODUCTION TO X TOOLKITS}

Xlib can be used to build applications, but its capabilities are basic and difficult to use. The $\mathrm{X}$ window system comes with the $x$ Toolkit Intrinsic (known as $x t$ Intrinsic) and a set of user interface components (known as widgets). The xt intrinsic can support many different widget sets. Both $\mathrm{xt}$ intrinsic and $\mathrm{x}$ widget are built on top of $\mathrm{xl}$ ib (See Figure 7). The $\mathrm{X}$ Widget set implements user interface components, such as scroll bars, menus, and buttons, while the xt Intrinsic provides a framework that allows the programmers to combine these components to produce a complete user interface. 


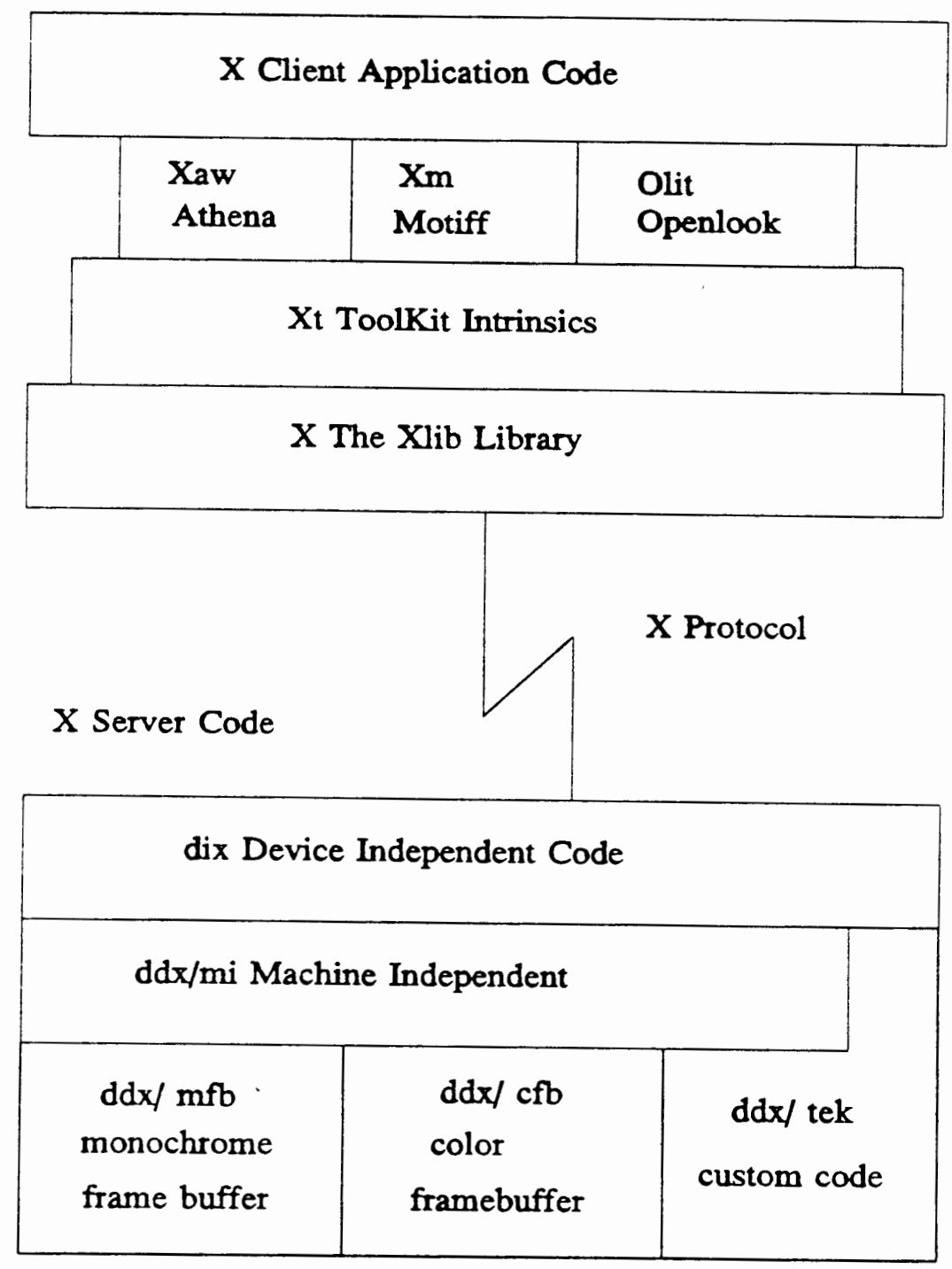

Figure 7. X Toolkits structure.

BASIC STEPS OF XT INTRINSIC PROGRAMMING

The Xt Intrinsic and $\mathrm{X}$ Widget set are smoothly integrated with XIib, so that the applications which use the additional 
facilities of higher level libraries can also use the functions provided by XIib.

widgets form a hierarchical structure which is similar to the $\mathrm{X}$ window tree structure, known as a widget tree. The root of every widget tree must be a special type of widget, known as shell widget which provides an interface between its child widget and the window manager. A shell widget must have exactly one child widget.

The steps of Xt Intrinsic programming are listed below:

1. Initiate the Intrinsic. This step establishes a connection to the $\mathrm{X}$ server, allocates resources, and initials the Intrinsic layer.

2. Create widgets. Every program creates one or more widgets to construct the program's user interface.

3. Register callbacks and event handlers. Callbacks and event handlers are application defined functions that respond to user actions within each widget.

4. Realize all widgets. Realizing a widget creates the $\mathrm{X}$ window used by the widget.

5. Enter the event loop. The event loop retrieves events from the $\mathrm{X}$ event queue and invokes a procedure, associated with the widget in which the event occurred, to handle the event.

At the beginning, the application must call the xtInitialize function before calling any other Intrinsic 
functions. Besides establishing a connection to the $\mathrm{X}$ server and initializing a resource database used by $X$ resource manager, it also creates and returns a Toplevelshell widget. This widget serves as a base for all other widgets in the application.

If a application uses multiple independent windows, an additional shell widget for each top-level window must be created. The xtcreatewidget function provides the way of creating all widgets except shell widgets.

Some widgets provide hooks that allow applications to define procedures which are called when some widget-specific conditions occur. These hooks are known as callback lists and the application's procedures are known as callback functions. Each widget maintains a callback list for each type of callback it supports. Callbacks are invoked by the widget rather that the Intrinsic, and are not necessarily tied to any particular events. Applications can add a callback to a callback list with xtAddCallback function.

The function xtRealizewidget creates a window for the widget. Once the xtRealizewidget is called for a particular widget, the widget and its children are said to be realized. 


\section{PROGRAM IMPLEMENTATION}

This chapter will provide an algorithm of our program. The program can be broken into four modules: working environment, image creation, image editing and image improvement. The program implementation and respective descriptions of these four models are in the following sections. Figure 8 is the structure of an $X$ window image display program.

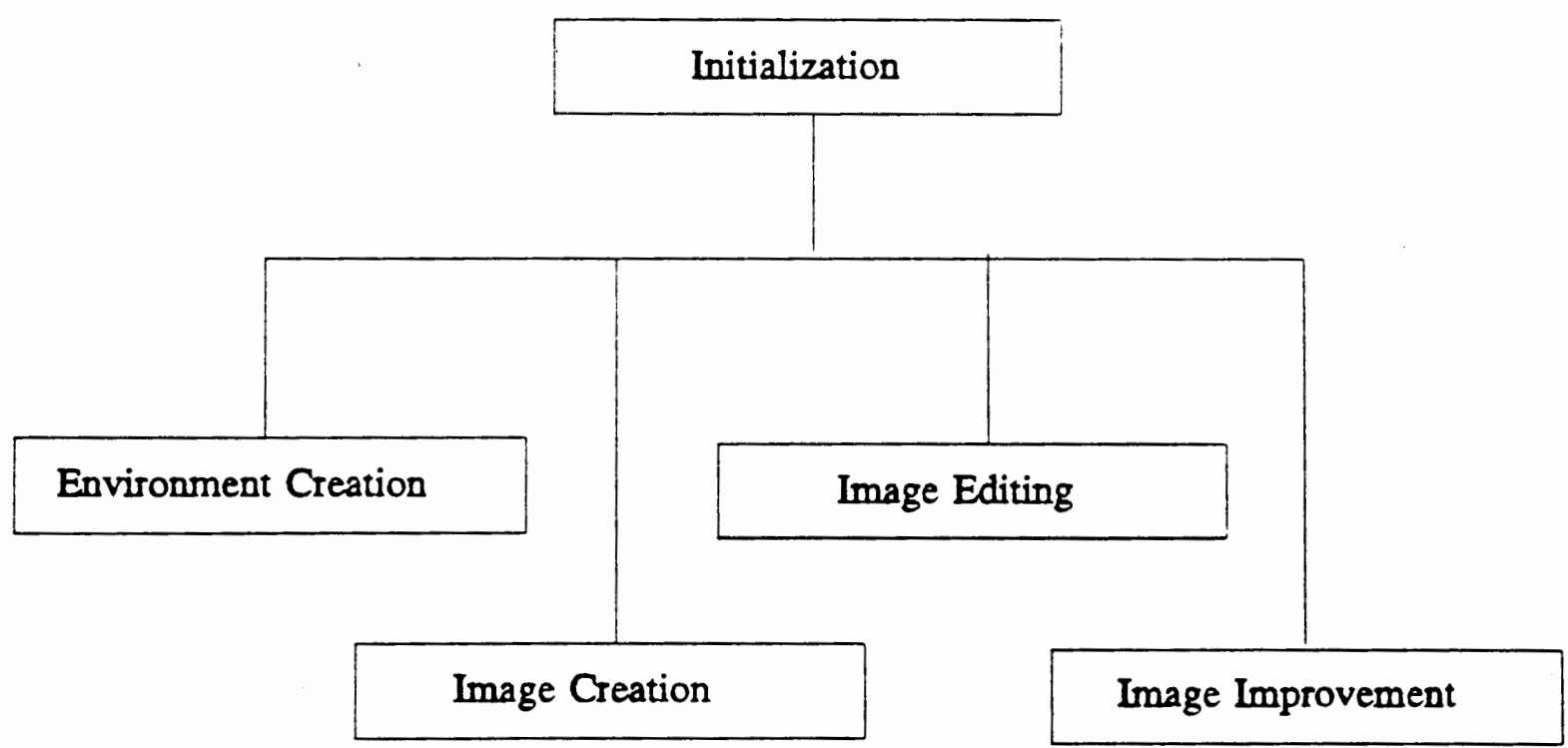

Figure 8. Program modules. 
Toplevel and Working Window Creation

The working environment model includes working windows, scrollbars, dialog box, menu buttons and mouse tracker. These are the widgets which are built by using $x t$ Intrinsic and widget Toolkits. The Figure 9 shows the widgets and the relationship among them.

\section{Toplevel}

(Toplevel Shell)

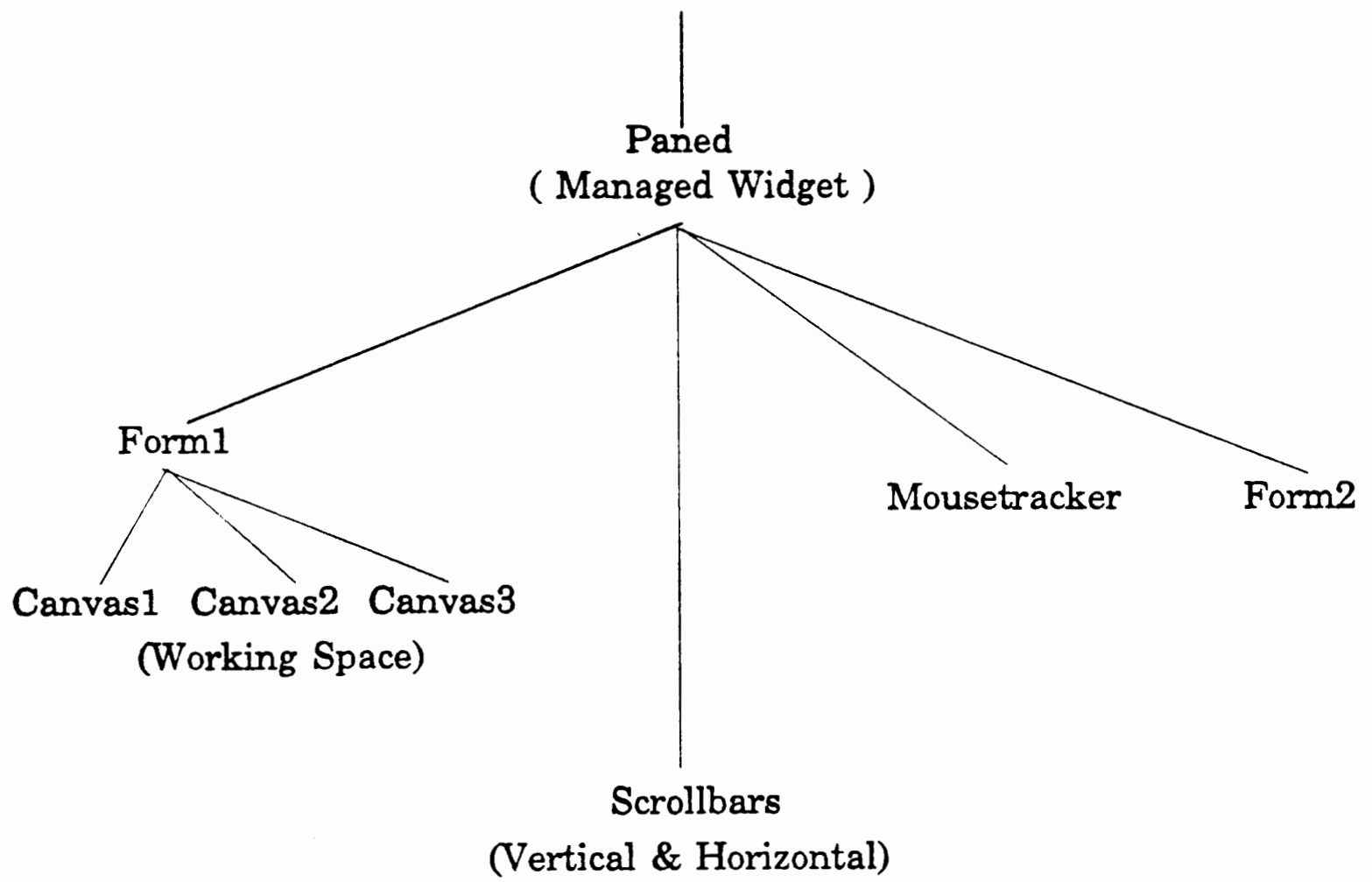

Figure 9. Widget tree structure. 
The toplevel widget is created at the procedure of initialization by using xtAppInitialize function which includes:

1. XtToolkitInitialize, which initiates the $\mathrm{x}$ Toolkit internals;

2. xtcreateApplicationcontext, which initiates the per application state;

3. XtDisplayInitialize, which initiates the per display state;

4. XtAppcreateshell, which creates the initial widget.

The resource data base file is loaded at the time when XtToolkit is initialized. It is defined as follow. static string fallback_resources[] $=\{$

"*input: TRUE",

"*allowShellResize: TRUE",

"*Dialogue.label: Input Format: Filename width height", "*Dialogue*label.resizable: TRUE",

"*Dialogue.value: ",

"*Dialogue*value.translations: \#override $\backslash \backslash n$

<Key>Return:Ok()" , "*scrollbar*orientation:

horizontal",

NULL, 
In this resource data file, it makes the dialogue box resizable and gives the first appeared label in the dialogue box. The horizontal scrollbar is also defined.

After the initial jobs have been finished, xtcreatemanagedwidget function can be called to create a composite widget to hold children widgets. It is a Paned widget type which can manage its children in a vertically or horizontally titled fashion. The panes may be dynamically resized by using the grips that appear near the right edge of the border. This widget is the toplevel framework.

Under the Paned Widget, a formwidget is created by calling xtcreatemanagedWidget function to hold three workspacewidgets and the scrollbar widgets. The reason of using formwidget here is that the workspace window sometimes needs to be reallocated. The Form provides geometry management for its children, which allows the individual control of the position of each child. When the Form is resized, it computes the new positions and sizes for its children.

The three workspacewidgets, canvasl, canvas2 and canvas3, are used to display images. The size of them can be changed by calling xtsetArg to match the different image data format types.

A pair of scrollbar widgets are also generated under the Paned toplevel widget. The scrollbars perform the function of image scrolling that allows the user to view the full image 
after the image zooming or image merging function has been executed.

In order to draw in a widget or perform other XIib functions, widget must be realized after the widget tree has been built up. When the toplevel widget is realized, all children widgets are realized also.

$X$ allows the reading and processing of an event anywhere in an application. Widgets in the $\mathrm{x}$ Toolkits neither directly read events nor grab the server or pointer. When an event or a class of event occur in that widget, it register procedures which are to be called. An application must consist of a startup code followed by an event loop that reads events and dispatch them by calling the procedures that widgets have registered. This default event loop is provided by XtAppMainLoop.

\section{Pull-down Menu Button and Dialogbox Creation}

The createFormwithButton which is called by main routine performs most of the interactive tasks, such as pull-down menu button creation and dialogbox creation. A number of subroutines are called by createformwithButton to obtain the image editing and image processing feature. The Figure 10 shows the tree structure of pull-down menu button and dialogbox widget tree.

The winselect function will choose the working window among the three (canvas1, canvas2 and canvas3). When a user clicks the window selection button, the winselect is called to 
setup particular working window flag to 1 and the rest of them to 0 . The value of the flag is stored as global parameter which will be used by c_image() to send an image into this selected window.

The dial() routine will create a user application dial box which is used to prompt for auxiliary input from user. The Dialogue widget has three areas. The first one is a description of a function. The second one is for the user input. The last one can be buttons which let the user confirm, cancel or choose different types of inputs.

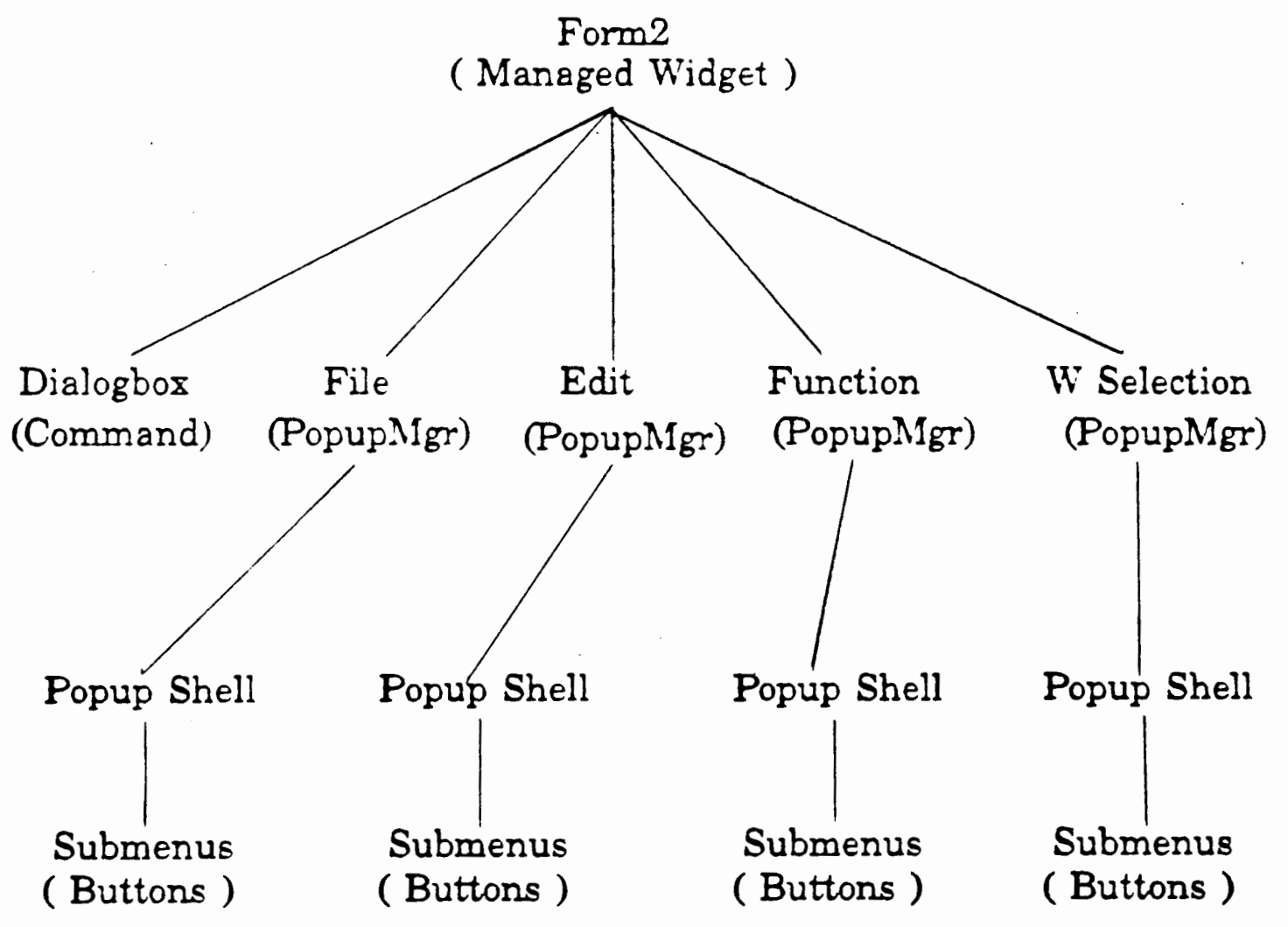

Figure 10. Pull-down menu buttons and dialogbox widget tree. 
The dialogbox can be created as popup type which means only when a user clicks the pop-up button the dialogue box will show on the screen. This type of widget must be the child of a pop-widget shell. The Intrinsic supplies popup shells which can simultaneously manage more than one child. Both the shell and child taken together are referred to as the popup $[10]$

In the dial(), the popup shell is created by calling xtcreatepopupshell. After the popup shell was created, the dialog widget can be created by calling xtcreatemanagedwidget function with dialwidgetclass. The button widgets, W1, W2, W3, FileInput, Rotation, Merge, Sceensave, Save, ImageTrl, ImageTr2, Cancel and ok, can be created under it as children of the popup manager. They appear on the third Iine of dialogbox widget.

When W1, w2 or W3 button is clicked, the w1(), w2() or w3() subroutine is called to set the window selection flag number. The functions of these three buttons are the same as the window selection buttons discussed before. Among the buttons of FileInput, Screensave, Save, Marge ImageTr1, ImageTr2 or Rotation, if one is clicked by a user, the respective routine will be called to change the prompt on the first line of the dialogbox and set the proper loop flag number. For example, if the FileInput button is clicked, the fileinput() routine is called to change the description which appears on the first line of dialog box to prompt the user of 
the input format. The loop_flag number is also set to 1 and passed to dialbox() which is called after the ok button is clicked.

The $O K$ button is used to confirm the user's request. A user has to click the $\mathrm{OK}$ button to execute the selected function. The corresponding routine of the ok button is dialbox(). If the cancel button is clicked, the DestroyPopupprompt() will be called to destroy the popup dialogbox.

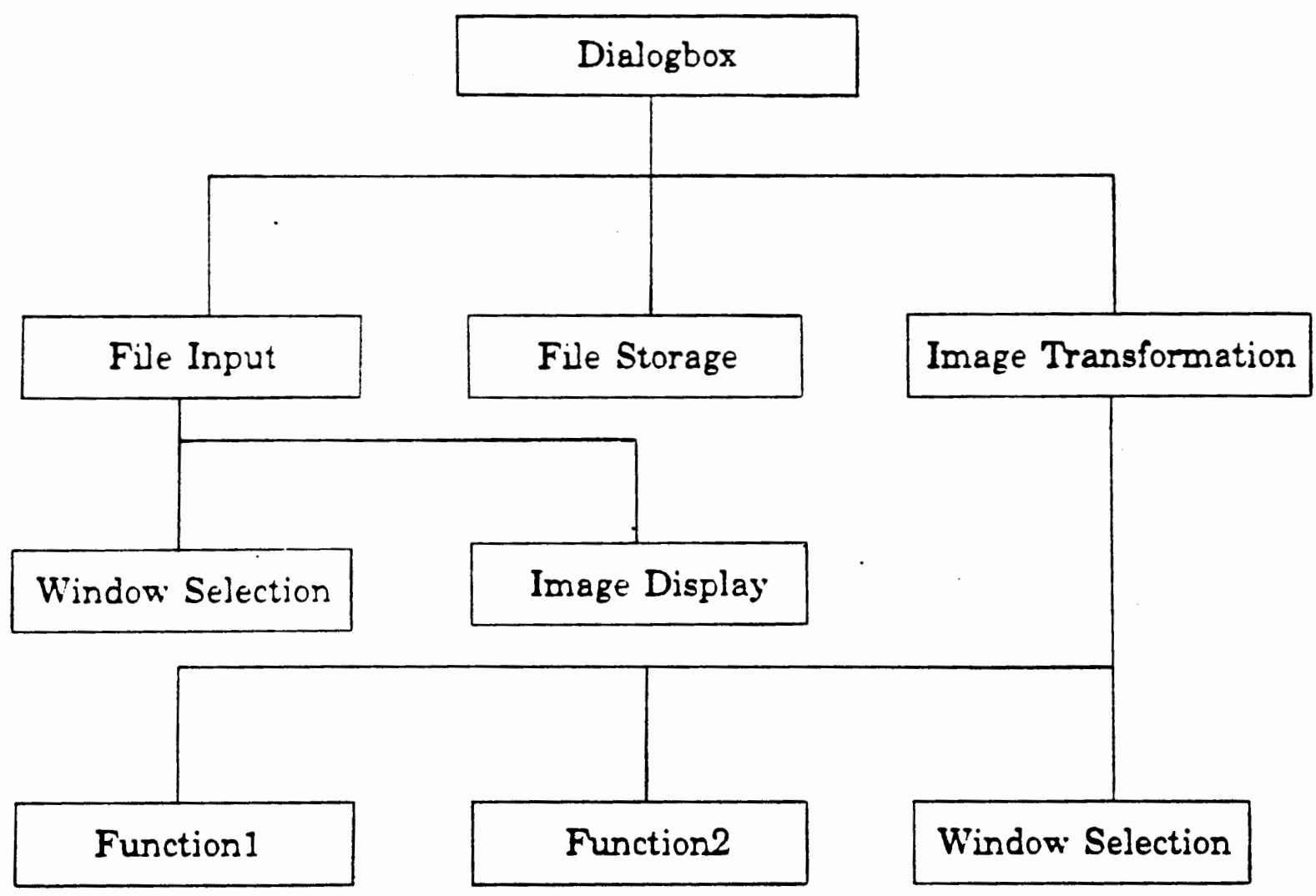

Figure 11. The dialbox routine structure. 
The dialbox() routine performs the most important part during dialog procedure. The Fig. 11 shows the structure of this routine.

Two widgets, dialog and button, are defined as widget type at the beginning of this routine. They are created in dial() routine when the dialog function is executed and passed into the dialogbox(). The character string in the text field of the dialog widget can be retrieved and saved by calling XawDialGetValuestring.

Several functions, FileInput, Sceensave, Save, Rotation, Merge, ImageTr1, ImageTr2, are be performed when the dialogbox() is called. All functions are controlled by the loop_flag number. It is assigned when a user clicks the function button in dialogbox. The loop_flag numbers are defined as following:

loop_flag=1, file input.

loop_flag=2, image transformation.

loop_flag=3, image transformation.

loop_flag=4, screen save.

loop_flag=5, file save.

loop_flag $=6$, image rotation.

loop_flag=7, image merging.

In the fileinput mode, the program first checks if the file exists. If the file does not exist, the program will go out of the dialbox() routine and change the description on the 
first field of the dialogbox and ask the user to input a file name again. Otherwise, the image data file name is stored in a temporary array. The name and widget are passed to both c_load() and c_data() routines. The data are retrieved into the data structure and the new color values are allocated in the c_data() routine. The last process of this mode is to send the new image to workstation screen by calling c_image().

When the loop_flag number is equal to 2 or 3 , the program enters the image transformation. The detailed explanation is in the next section.

The Screensave mode saves the image displayed on the screen to a sun.rasterfile type data file. At the first step, each color value of the image will be searched to find out the corresponding pixel value according to the colormap. In the second step, the sun.rasterfile header, colormap and image pixel values are saved to an image data file with the type of sun.rasterfile.

The Save mode is different from the Screensave mode. It saves the image pixel values stored in the data structure rather than the pixel values corresponding to the screen color values. Two different sizes of image may be stored in the data structure: the window size which is 256 by 256 or larger size 512 by 512. They are distinguished by the value of header_flag. If the size is 512 by 512, the header-flag is equal to 1 , which is passed from the image zooming or the image merging routine, otherwise the header_flag is equal to 
0. In the case of the larger size, the two fields of the sun.rasterfile header, header.ras_wide and header.ras_height, are set to 512. Therefore, the saved image data file has the size of 512 by 512 .

In the Merge mode, the character string in the user input field of dialogbox is separated and saved as four names. They can be either a window name or an image data file name. If the name indicates a window, the corresponding data of that window will be copied. Otherwise, the data file will be opened and read by calling $c_{\text {_lload( }}$ (lond c_data(). The scrollbar may be used to allow the user a full view on the merged image since the merged image is four times as big as the window size. The herder_flag is set to 1 .

In the Rotation mode, the angle is read in from the user input field of the dialogbox and passed to rotate() routine. The image rotation function is accomplished by calling rotate ().

The buttons, File, Edit, Function, are also created by CreateformwithButton(). These are the pull down menu buttons with several entries in each of them, such as image zooming and image rotation buttons are under the pull-down menu button named Edit. For the $\mathrm{X}$ window programming, the pull-down menu can be brought out easily by calling $x$ widget functions. We first create a popup managed widget with the class of menuButtonwigetclass. Then, we create the pop-up shell with the simplemenuwidgetclass under that button. This is used to 
manage all it's children with pop-up feature. All the function call entries are created with smbBSBObjectclass widget class. These can be seen from Figure 10.

\section{IMAGE CREATION MODULE}

This model has the functions of building colormap, loading image data file, converting pixel values and sending image to display. These functions are accomplished by calling c_colormap(), c_load(), c_data(), c_image() routines. The function of each routine is described below:

In the c_colormap(), a colormap will be built with 128 color cells. $X$ converts the data pixel values to the screen color values according to this colormap. The colormap can be shared by all windows.

c_load() loads data file. The program first read in the header of data file and checks the field of ras_magic. If it is $0 \times 59 a 66 a 95$, it indicates that the data file is a standard sun.rasterfile. The header is stored into the header structure. According to the information of this header, colormap and data are read into colormap and data structure separately. If the ras_magic is not 0x59a6695, the program assumes that the data file is a plain data file with 256 width and 256 height. The proper values are assigned to each field of the header structure and the colormap, which was built during the building colormap procedure. The sun.rasterfile header format is as follows. 


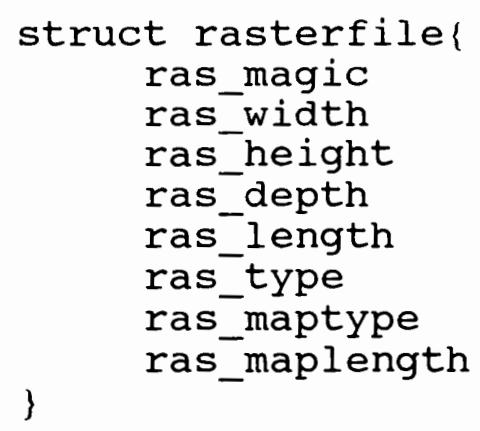

For a sun.rasterfile, the ras_magic is always 0x59a66a95. The ras_width and ras_height and ras_depth fields contain the image's width and height in pixels, and its depth in bits per pixel, respectively. The depth is either 1 or 8 , corresponding to the standard frame buffer depths. The ras_length field contains the length in bytes of the image data. The fields ras_maptype and ras_maplength indicate type and length of colormap. If the ras_maptype is not RMT_NONE and the ras_maplength is not 0 , then the colormap values are the ras_maplength bytes immediately after the header.

c_data() routine performs the function of data transfer. Each pixel value is the index of colormap. The corresponding color values can be found by checking at the colormap. The converted values are stored into the data file of image structure.

In the c_image routine, the fields of image data structure are filled to prepare a displayed image. A corresponding GC is also generated. Then, the XPutImage function is called to send the image to workstation display. 
Each window has its own data and image structure. The C_load(), c_data() and c_image() routines must be repeatedly called three times to fill three window's data structure when the application starts. During the executing procedure, the data structure of three windows can be changed individually when a user accesses the image improvement function or inputs a new image using the dialogbox.

\section{IMAGE EDITING MODULE}

Two functions are contained in this model: image zooming and image rotation.

\section{Image Zooming}

Image zooming includes two functions: zoom in and zoom out.

When an image is to be magnified by an integer zoom factor, pixel estimation can be efficiently implemented by convolution. For example, consider an image magnification by a factor of $2: 1$. This operation can be accomplished in two stages. First, the input image is transferred to an array in which the rows and columns of zeros are interleaved with the input image as shown below:
A $B$
$\begin{array}{llll}0 & 0 & 0 & 0\end{array}$
C D
$\begin{array}{llll}C & 0 & D & 0\end{array}$
$\begin{array}{llll}0 & 0 & 0 & 0\end{array}$ 
At the second step, the zero interleaved neighborhood image is convoluted with the discrete interpolation kernels Peg.

\begin{tabular}{|c|c|c|c|c|}
\hline & & $\mathrm{A}$ & $A$ & B \\
\hline$I$ & 1 & $A$ & $A$ & B \\
\hline 1 & 1 & $C$ & C & $\mathrm{D}$ \\
\hline & & $\mathrm{C}$ & $C$ & $\mathrm{D}$ \\
\hline
\end{tabular}

Kernel: Peg

Result of Magnification

The result of this image magnification is 4 times larger than the original image. This function can be executed repeatedly, but the resolution of the magnified image will be lower than before.

According to the image magnification method we mentioned above, the pixel values are computed to generate a new data array width four times as big as the original one. Then, one quarter of this data is sent to a certain working window to display by calling the c_image() routine. If the user selected a point by clicking the mouse button on the window screen before this image magnification operates. The part of new magnified image displayed in the window will be centered at that point. If there is no value input, the part of a new magnified image will be started from the upper-left corner with the window size of width and height.

The zoom out is a useful function to view a merged image or reset a magnified image. It is the opposite of zoom in. 
Mouse cursor point recognition and Image scrolling

Once an image has been magnified, the size of it is four times as large as the original one (in the case of zooming once). In order to get a full view of this image on the same window, we add the image scrolling function in the program. This function can be accomplished by combing some subroutine calls, c_mouse_track(), scrolledv(), scrolledh() and move().

The function, mouse cursor point recognition, allows the user to select the working window or to get the mouse cursor position in the window. The routine c_mouse_tracker() performs this function. It reads the mouse position on the screen and stores it in $x$ event structure. Anytime when the image scrolling is requested, the $x$ and $y$ position values of the mouse curser are retrieved from $\mathrm{x}$ event structure and added to the values which are generated by scroll bar. These fixed values are passed to the move() routine to accomplish the image scrolling feature.

To move an image, the value passed from c_mouse_track() is chosen as the center address of the displayed area in the image data matrix. The upper-left corner address can be very easily counted through the function below:

$$
i=p x-\frac{1}{2} * \text { width }, \quad j=p y-\frac{1}{2} * h e i g h t
$$

Where px and py values are passed from c_mouse_track() routine, the $i$ and $j$ are new addresses of the upper-left corner pixel $\mathrm{x}_{(\mathrm{i}, \mathrm{j})}$. With the upper-left pixel address, width and 
height, this sub-image can be extracted from the magnified image and be sent to the display window by calling c_image routine.

Image Rotation

Rotation model performs the function of image rotation. Rotation of an input image about its cartesian origin can be accomplished by the address computing and mapping. To prevent the holes in destination image, the reversed coordinates transformation method is applied. The following equations show mapping the image pixels from destination to source.

$$
\begin{aligned}
& \mathrm{V}_{\mathrm{x}}=\mathrm{U}_{\mathrm{x}} \cos \theta+\mathrm{U}_{\mathrm{y}} \sin \theta \\
& \mathrm{V}_{\mathrm{y}}=\mathrm{U}_{\mathrm{y}} \cos \theta+\mathrm{U}_{\mathrm{x}} \sin \theta
\end{aligned}
$$

The $\theta$ in the equations is counter-clock wise angle of rotation with respect to the horizontal axis of the input image. It was input by the user through dialogbox. $U_{x}$ and $U_{y}$ are the destination address. $V_{x}$ and $V_{y}$ are the source image pixel address. The image can be rotated around any point in the window screen. The origin is picked by the user clicking the mouse on a point. If no origin is chosen, the image will rotate around the center point of the window. The rotated pixel values are stored back to the image data structure. 
IMAGE IMPROVEMENT MODULE

This model performs the image transformation and some image enhancement functions.

\section{Image Point Transformation}

The image point transformation model can be used to change the pixel levels in an image when the user requires it. This model includes two sub-models, ImageTrl and ImageTr2. Both of them are with some image point transformations. The structure of this model is shown in Figure 12.

\section{Input Parameters}

Input Image $\longrightarrow$ Transfer function

Output Image

Figure 12. Image point transformation module.

In ImageTrl, two parameters are read in from the text field of the dialogue box. The program will check both the sign and value of them to perform the different mapping function. The corresponding of parameters and functions can be seen from the following: 
Parameters

A B

$-A \quad-B$

$0 \quad-B$

$-A \quad O$
Functions

Binary window

Inverse Binary window

Threshold

Inverse Threshold
Mapping Graph
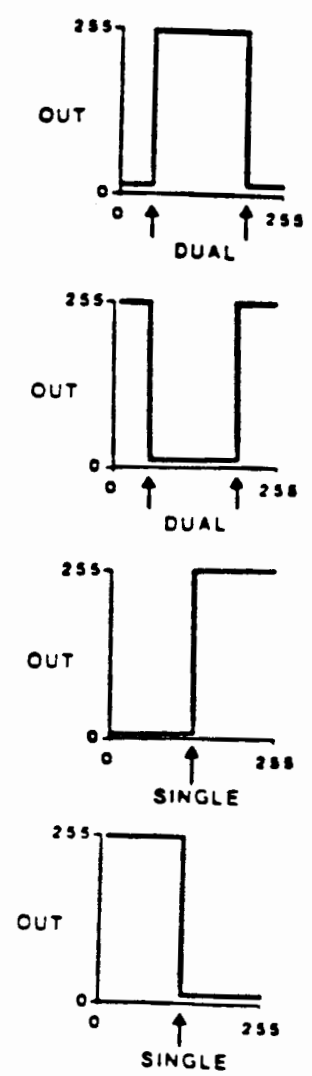

Similarly to the ImageTr1, the ImageTr2 just has more conditions and has to be calculated through an equation. After the value is calculated, the result is compared with two thresholds $L$ and $H$. The corresponding of function and parameters can be shown below:

Transfer Equation:

$$
Y=A *(X+B)^{C}+D
$$


Parameters

$A, \quad B=0, C=1, \quad D=0$

$I=0, \quad H=255$

$-A, \quad B=-255, C=1, \quad D=0$

$I=0, \quad H=255$

$A, \quad B=0, C=1, \quad D=0$

I, $\mathrm{H}$

$-A, \quad B=-255, C=1, \quad D=255$

$\mathrm{L}, \mathrm{H}$

A, B, $C=1, D=0$

$-\mathrm{L}, \mathrm{H}$

$A, B, C=1, D=0$

$I,-H$
Functions

Linear

Inverse Linear
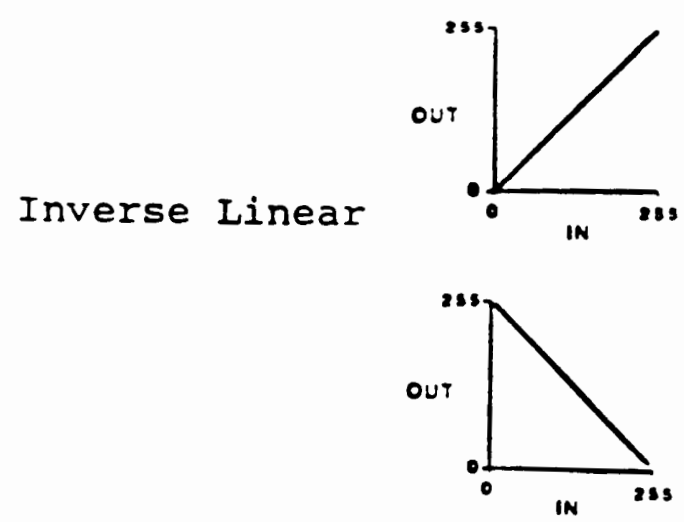

Suppress

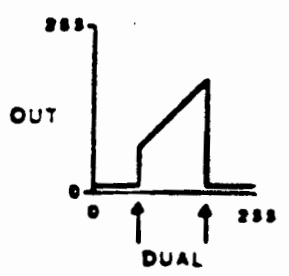

Inverse suppress

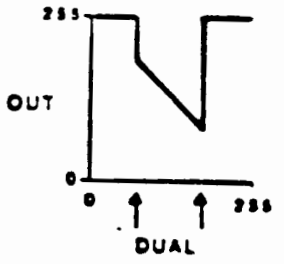

Suppress Window

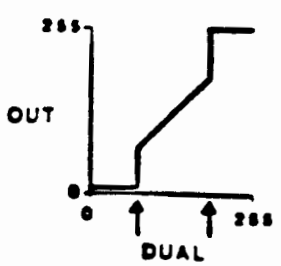

Inverse Suppress Window

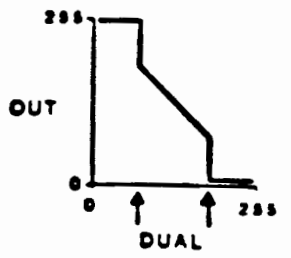

$A, B, C=1, D$

stretch

L, $\mathrm{H}$

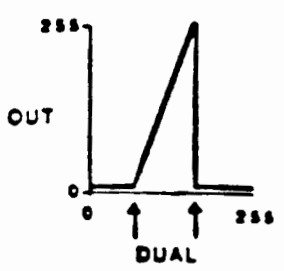


Image Enhancement

This model includes some image enhancement functions. They can be executed by user clicking on the pull-down menu buttons. The button 'FUNCTION' is generated in createformwithButton() routine. The pull down menu has several entries: Noise, Media, Ave, Histo, Sharp, Direct, Diff and Inverse. Each entry is associated with a button on the menu. When a selected button is clicked, the corresponding subroutine is called. This section will discuss the main functions of these subroutine functions.

histo() is a subroutine which performs the function of HISTOGRAM MODELING. The histogram of an image represents the relative frequency of occurrence of the various gray levels in the image. Histogram-modeling techniques modified image so that its histogram has a desired shape. This is useful in stretching the low-contrast levels of images with narrow histograms.

In histogram equalization, the goal is to obtain a uniform histogram for the output image. Let the variable $r$ represent the gray level of the pixels in the image to be enhanced. The transformations of the form

$$
s=T(r), a \leq r \leq b
$$

produce a level $s$ for every pixel value $r$ in the original image. It is assumed that the $T(r)$ satisfies the conditions: 
1. $T(r)$ is a single-valued and monotonically increasing in the range of $a \leq r \leq b$.

2. $a \leq T(r) \leq b$ for $a \leq r \leq b$.

Considering an image pixel value $r$ in the ranges of $(0$, 255) with a continuous probability density function $P_{r}(r)$ and cumulative probability distribution s. then, the random variable

$$
S=T(r)=\int_{0}^{r} P_{r}(w) d w \quad 0 \leq r \leq 255
$$

will satisfy the two conditions and is uniformly distributed.

In discrete type, the probabilities of pixel gray levels can be given by the relation

$$
P_{r}\left(r_{k}\right)=\frac{n_{k}}{n} \quad 0 \leq r_{k} \leq 255, \quad k=0,1, \ldots, L-1
$$

where $P_{x}\left(r_{k}\right)$ is the probability of the kth gray level, $n_{k}$ is the number of times this level appears in the image, and $n$ is the total number of pixels in the image. The plot of $P_{Y}\left(r_{k}\right)$ versus $r_{k}$ is usually called a histogram.

The discrete form of transformation can be obtained by the following equation

$$
\begin{array}{rlrl}
s_{k} & =T\left(r_{k}\right)=\sum_{j=0}^{k} \frac{n_{j}}{n} \\
& =\sum_{j=0}^{k} P_{r}\left(r_{j}\right) & 0 \leq r_{k} \leq 255, \quad k=0,1, \ldots, L-1,
\end{array}
$$

once the transformation function was found, we can map the pixel values of the original image into desired new values according to this $s_{k}$ series. Since only 256 equally spaced 
levels are allowed in our case, each of the transformed values must be assigned to its closet valid level. The new image may has less pixel values.

media() is the routine with the Median filtering function. Median filter is a nonlinear signal processing technique developed by Tukey [5]. It is very useful for noise suppression in images. The input pixel is replaced by the median of the pixels contained in a window around the pixel, that is,

$$
V(m, n)=\operatorname{median}(U(m-k, n-1)), \quad(k, 1) \subseteq W
$$

where $W$ is a suitably chosen window. Typical windows are $3 \times 3,5 \times 5,7 \times 7$ or the five-point window. The algorithm for median filtering requires arranging the pixels in the window in increasing or decreasing order and picking the middle value. For example, the values of the pixels within a $3 \times 3$ window are $1,3,5,2,10,6,4,8,7$, the center values would be 5 after sorting in increasing order $1,2,3,4,5,6$, $7,8,10$. The center value 5 will replace the old value 10 . In this example, if the value 10 were a noise spike in a monotonically increasing sequence, the median filter would result in considerable improvement. Likewise, this window will slide all over the image data to approach the noise suppression. On the other hand, it may also cause signal suppression. 
We applied a $3 \times 3$ two dimensional median filter on Lenna's image picture. Lenna's picture was added by the GAUSSIAN noise on all pixels. The result of filtered image was improved significantly although some blurs still can be seen. The cascade median filter provides a greater degree of noise suppression, but it also results in greater signal suppression.

ave() routine performs the function of neighborhood averaging. This is the spatial-domain low-pass filtering. Given a $\mathrm{N} \times \mathrm{N}$ image $\mathrm{U}(\mathrm{x}, \mathrm{y})$, the procedure is to generate a smoothed image $\mathrm{V}(\mathrm{x}, \mathrm{y})$ whose gray level at every point $(\mathrm{x}, \mathrm{y})$ is obtained by averaging the gray-level values of the pixels of $\mathrm{V}$ contained in a predefined neighborhood of $(x, y)$. In simple words, each pixel is replaced by a weighted average of its neighborhood pixels, that is:

$$
V(m, n)=\sum_{(k, 1) \leq W} \sum a(k, 1) U(m-k, u-1)
$$

Where $U(m, n)$ and $V(m, n)$ are the input and output images, $\mathrm{W}$ is a suitable chosen sliding window, and $a(k, 1)$ is the filter weights. In common case, all weights are equal, giving

$$
V(m, n)=\frac{1}{N_{w}} \sum_{(k, 1) \leq W} \sum U(m-k, n-1)
$$

Where $a(k, I)=\frac{1}{N_{W}}$ and $N_{W}$ is the number of pixels in the window $\mathrm{W}$. 
Spatial averaging can be used for noise smoothing, lowpass filtering, and subsampling of images [4]. Suppose the observed image is given as

$$
V(m, n)=U(m, n)+\eta(m, n)
$$

Where $\eta(m, n)$ is white noise with zero mean and variance $\sigma^{2}$. Then the spatial average of above equation yields

$$
V(m, n)=\frac{1}{N_{W}} \sum_{(k, I) \leq W} \sum U(m-k, n-1)+\bar{\eta}(m, n)
$$

where $\bar{\eta}(m, n)$ is the spatial average of $\eta(m, n)$. It shows that $\bar{\eta}(m, n)$ has zero mean and variance ${\overline{\sigma_{\eta}}}^{2}=\frac{\sigma_{\eta}{ }^{2}}{N_{W}}$, that is, the noise power is reduced by a factor equal to the number of pixels in the window $W$. If the noiseless image $U(m, n)$ is constant over the window $\mathrm{W}$, then spatial averaging results in an improvement in the output signal-to-noise ratio by a factor of $N_{W}$. For example, a 3 x 3 sliding window is used in our program. According to the above, $N_{W}$ is 9 . Then the averaging filter is given by

$$
V(m, n)=\frac{1}{9} \sum_{(k, 1) \leq W_{3}} \sum U(m, n)+\frac{\sigma_{\eta}^{2}}{9}
$$

The noise power is reduced by 9 . By testing the same Lenna's picture, the result of noise filtering is obvious.

direct() routine is developed from ave(). To protect the edges from blurring while smoothing, a directional averaging filter is employed. The algorithm of this routine is similar 
to the ave() routine. But, it calculated the average of the window in several directions.

$$
V(m, n: \theta)=\frac{1}{N_{\theta}} \sum_{(k, I) \leq W_{\theta}} \sum U(m-k, n-I)
$$

The direction $\theta^{*}$ is found such that $\left|U(m, n)-V\left(m, n: \theta^{*}\right)\right|$ is minimum. Then $V(m, n)=V\left(m, n: \theta^{*}\right)$ gives the desired result. comparing the resultant directional averaging with an averaging filter, it has a clearer picture and better edges. The directional averaging filter is shown as Figure 13 .

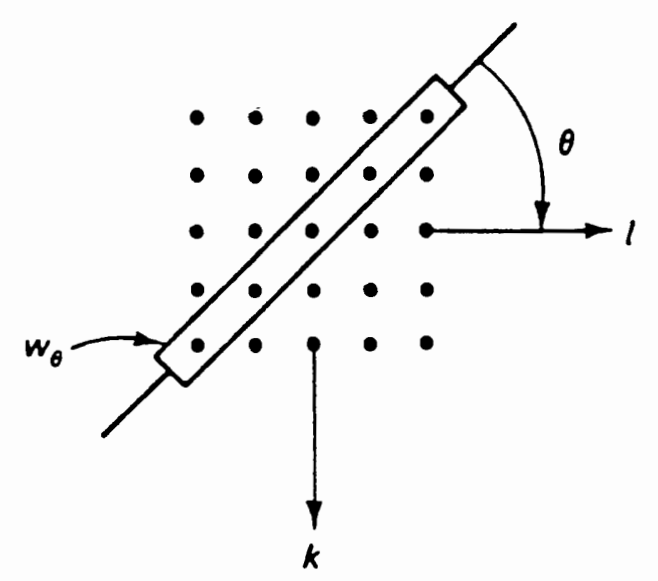

Figure 13. Directional averaging noise filter.

sharp() routine can be used to crispen the edges of an image. A signal proportional to the unsharp, or low-pass filtered, version of the image is subtracted from the image. This is equivalent to adding the gradient, or a high-pass signal, to the image ( See Figure 14). 


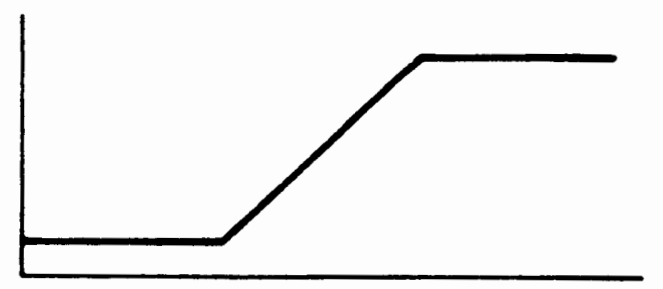

Signal

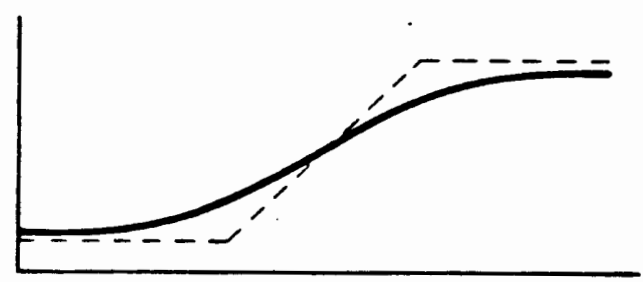

\section{Low pass}

(1) $-(2)$

High.pass

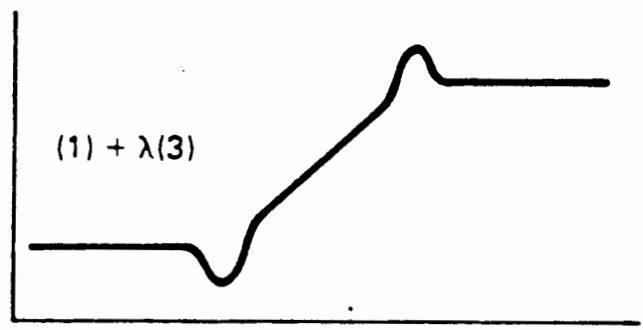

Figure 14. Waveforms in an unsharp masking image enhancement system.

The unsharp masking operation can be represented by

$$
V(m, n)=U(m, n)+\lambda * g(m, n)
$$

where $\lambda>0$ and $g(m, n)$ is a suitably defined gradient at $(m, n)$. The radiant function is the discrete Laplacian

$$
g(m, n)=U(m, n)-\frac{1}{N} \sum_{(k, 1) \leq W_{N}} \sum U(m-k, n-1)
$$


The result of using the Laplacian to operate on Lenna's image shows that the masked signal has a longer duration edge gradient as well as an overshoot and undershoot as compared to the original signal. Subjectively, the apparent sharpness of the original image is improved. The sharpening effect is stronger as the mask window size $\mathrm{N}$ increases. 
CHAPTER V

CONCLUSION

IMPROVEMENT AND FUTURE WORK

The combined functions of Xlib and X Toolkits can give us a lot of new features. It saves several hundred line codes on dealing with window manager, scroll bars and pull down menus. Based on the XIib and Xt intrinsic, we developed an image display software comprising three separate working windows and several pull down menus. The software also has the function to improve images, such as image transformation, image enhancement and noise filtering.

Due to the restriction of the system hardware, the color display is much harder. One way to display a color image is to do quantization on the original image file.

The color image quantization task can be broken into four phase [3]:

1. Sampling the original image for color statistics.

2. Choosing a colormap based on the color statistics.

3. Mapping original colors to their neighbors in the colormap.

4. Quantizing and redrawing the original image (With optional dither). 
A typical form of a color image is usually a rectangular array of pixels each having 24 bits( 8 bits per component). Each color component is represented by numbers in the range 0 to 255. Color image quantization is the process of selecting a set of colors to represent the color gamut of an image, and computing the mapping from color space to representative colors. There are two general classes of quantization methods: uniform and tapered. In uniform quantization, the range of the input variable is divided into intervals of equal length. In tapered quantization, intervals are usually based on the statistical distribution of the input variable.

When quantizing to very few colors, the contouring can be visually distracting. The image which suffers from contouring can be improved with the technique of dithering. The basic strategy of dithering is to trade intensity resolution for spatial resolution. By averaging the intensities of 6 several neighboring pixels one can get colors not represented by the colormap. If the frame buffer is high enough, the eye will do the spatial blending for us. Taking advantage of this, is possible to reproduce many color images using only a few colors [3]. The Unix system command fbquant, uses tapered method. It should be possible to combine fbquant with dither dither strategy to accomplish the color quantization. The quantized image file with only 256 gray level then can be displayed on an 8 bits frame buffer workstation. 
Since the limitation of our SUN workstation system hardware(we have 8-bit frame buffer SUN workstations), the color image display looks rough when we simply scaled bright level of color image data file for the 256 entry of colormap. The next step of our goal is using the color quantization method to reduce the number of color levels and represent the color information of the picture on the screen.

\section{OTHER SYSTEM STUDY AND COMPARISON}

In this project, we also study and compare some other window or image processing system with $\mathrm{x}$.

SUN Pixrect package is with a set of Rasterop routines. With these routines, application programs can be written that access the display on all sun products. Pixrect library is a low-level package, sitting on top of the device drivers. The high-level graphics packages like sunview is also needed to help more complicated image application (e.g. for window system and input device support). Compare Pixrect to the $\mathrm{X}$ Window system, $\mathrm{x}$ Window system has the greater advantage since its network transparency, concurrency and vendor and model independent, the detail are reviewed in chapter II. 


\section{REFERENCES}

[1] Barkakati, Nabjyoti, "X Window System Programming," North College, Carmel, Indiana, 1991.

[2] Castleman, Kenneth R., "Digital Image Processing," Prentice-Hill, Englewood Cliffs, NJ. 1989.

[3] Gettys, J., Scheifler, R. W., Newman, R., "Xlib - C Language Interface," 1989.

[4] Gonzalez, Rafal C, Wintz, Paul, "Digital Image Processing," Addison-Wesley publishing company, 1987.

[5] Jain, Anil K, "Fundamentals of Digital Image Processing," Prentice-Hill, Englewood Cliffs, NJ. 1989 .

[6] Jones, Oliver "Introduction to the $\mathrm{X}$ Window System," Prentice-Hill, Englewood Cliffs, NJ. 1989.

[7] Mccormack, J. Asente, P.,Swick, R. R., "X Toolkit Intrinsics C Language Interface," 1989.

[8] Peterson, C. D., "Athena Widget Set - C Language Interface," 1989.

[9] Pratt, William k., "Digital Image Processing," 2nd ed., Wilwy \& Sons, inc., 1991.

[10] Young, Douglas A, "X Window Systems Programming and Applications with $x t, "$ Prentice-Hill, Englewood Cliffs, NJ. 1889. 
APPENDIX A

PROGRAM: XSHOW.C 


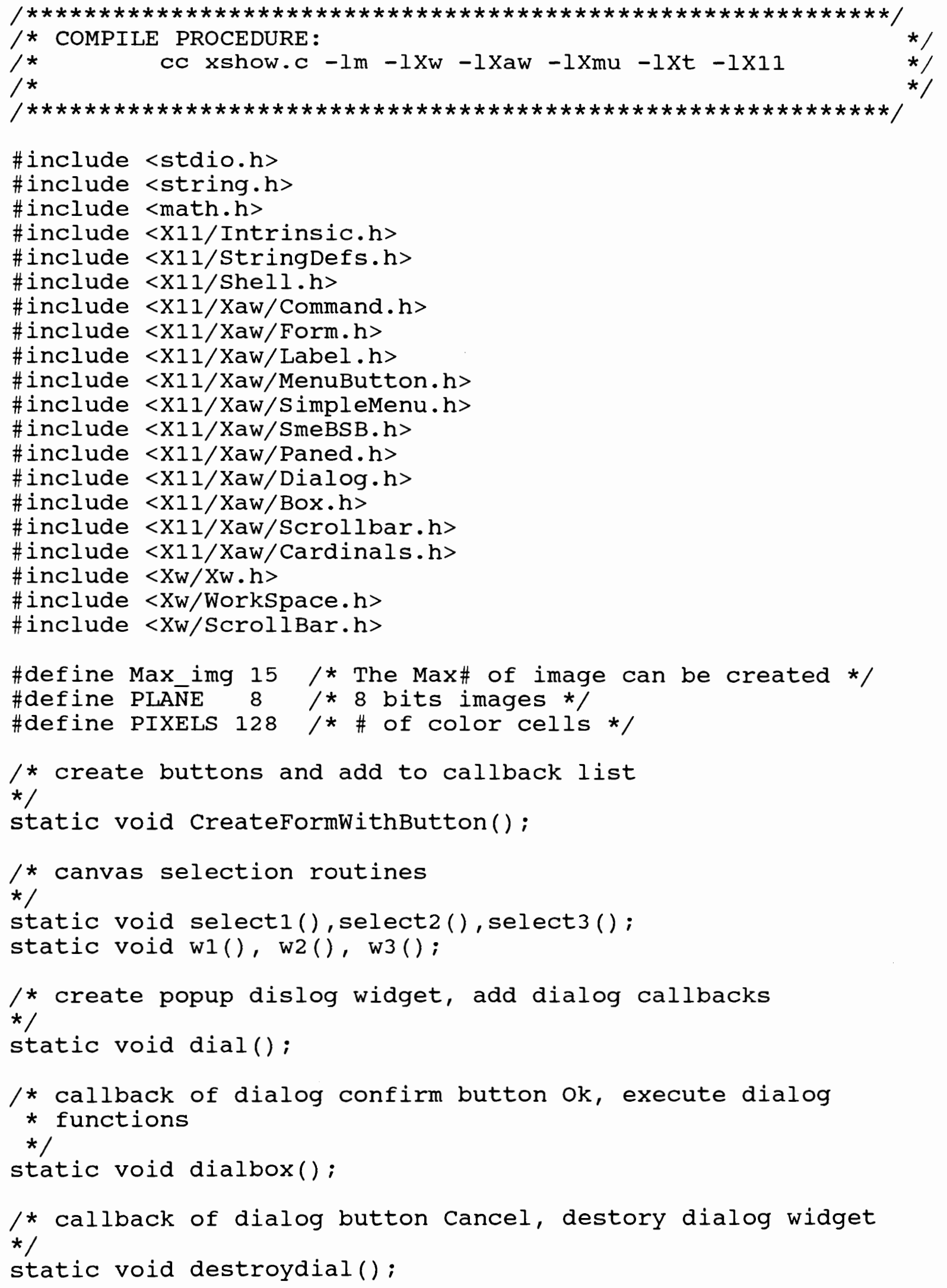


/* callback of merge button, performs the image merge function *

static void merge();

static void merge_count();

/* callback of Save button, performs the image saving function *

static void save();

/* callback of Screensave button, performs screen capture

* function

* I

static void save1();

/* callback of Input button, performs image loading function */

static void fileinput();

/* routine used to refresh window

*/

static void redisplay();

/* reload image

* /

static void restore();

/* rotate image

*

static void angle();

static void rotation();

/* image zooming

*/

static void enlarge();

static void reduce();

/* image processing routines

static void imagetr1(), imagetr2();

$s t$ a $t$ i $c$ v o $i d$ m e d i a ( )

histo (), sharp (), direct (), diff(), inverse ();

/* callback of Quit button, exit

*

static void Quit();

/* callback of popup Cancel button, destory popup dialog box */

static void DestroyPopupPrompt();

/* callback of dialog confirm ok button. excute dialog function 
Img_d mydata [3];

/* sun.rasterfile type image data header */

typedef struct rasterfile

$\begin{array}{lll}\text { int ras_magic; } & / * \text { ox59a66a95 */ } \\ \text { int ras_width; } & / * \text { image width } * / \\ \text { int ras_height; } & / * \text { image height } * / \\ \text { int ras_depth; } & / * \text { bits per pixel } * / \\ \text { int ras_length; } & / * \text { length of image data in bytes } * / \\ \text { int ras_type; } & / * \text { image data type } * / \\ \text { int ras_maptype; } / * \text { colormap type } * / \\ \text { int ras_maplength; } / * \text { colormap length } * /\end{array}$

\}rasterfile;

rasterfile head;

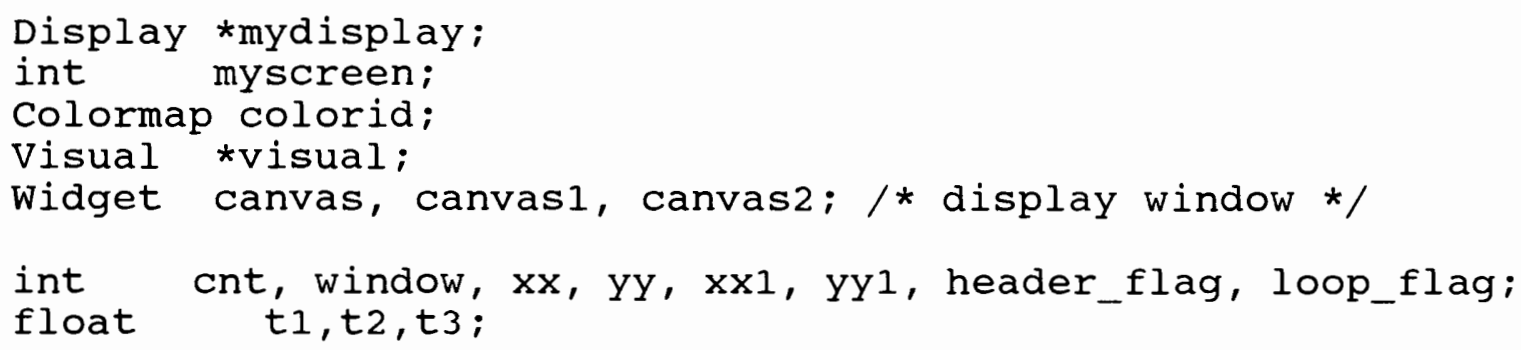


$t_{1}=($ float $) \cos (($ float $) 45 * 3.14159 / 180) ;$

t2 $=($ float $) \sin ($ (float) $45 * 3.14159 / 180) ;$

/* initate Xt Toolkit, create toplevel shell, load

* resource database */

toplevel = XtAppInitialize(\&app_con, "Popup", NULL, ZERO, \&argc, argv, fallback_resources, NULL, ZERO);

/* check input syntax */

if (argc $!=2)$

Syntax(app_con, argv[0]);

/* load action table resource */

XtAppAddActions (app_con, actionTable,

xtNumber (actionTable) ) ;

/* create management widget for children */

outer = XtCreateManagedWidget ("paned", panedWidgetClass, toplevel, NULL, ZERO);

/* create form type management to hold canvas widgets */ form 1 = XtcreateManagedWidget ("form", formWidgetclass, outer, NULL, ZERO);

width $=$ img [0] image. width;

height=img [0] . image. height ;

/* set canvasl width and height */

xtsetArg(wargs [n], XtNwidth, width) ; ++ ;

xtsetArg (wargs[n], xtNheight, height) in++;

/* create canvaso widget to display image */

canvas =XtcreateManagedWidget ("canvas",

Xwworkspacewidgetclass, forml, wargs, n) ;

/* set width and height of canvasl, locate at right of * canvaso

*/

XtSetArg(wargs $[n], x$ tNwidth, width) $i n++$;

xtsetArg (wargs $[n]$, XtNheight, height) in+t;

xtsetArg (wargs $[n]$, XtNfromHoriz, canvas) in++;

/* create canvas 1 widget to display image */ canvas 1 =xtcreateManagedWidget ("canvas1",

Xwworkspacewidgetclass, forml, wargs, n) ;

/* set width and height of canvas2, locate at right of

* canvas 1 


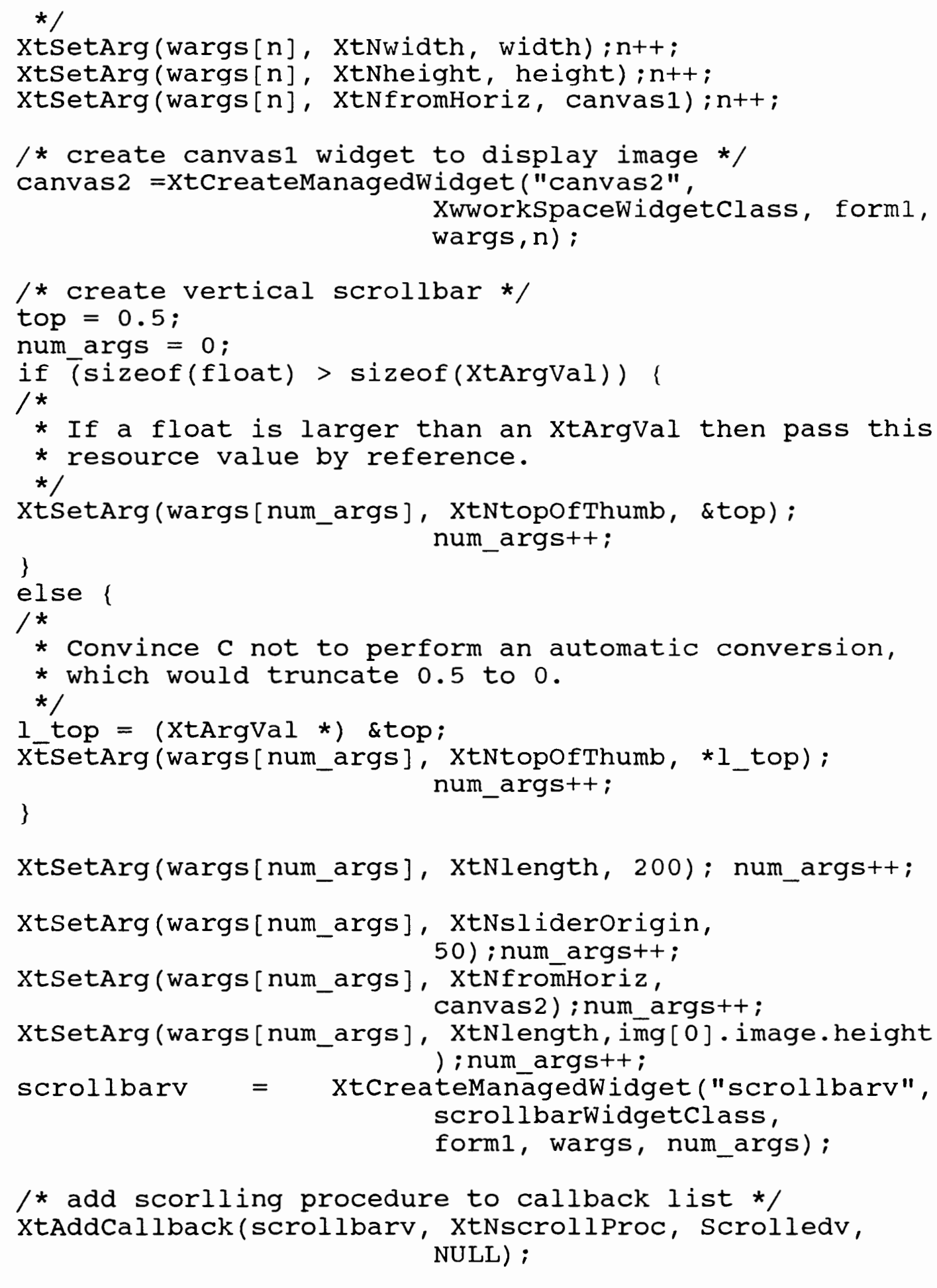

/* create horizontal scrollbar */

xtsetArg (wargs [num_args], xtnsliderorigin, 50) ; num_argst+;

XtSetArg (wargs[num_args], XtNlength, img[0].image.width ) inum_argst+; 
scrollbar = XtcreateManagedWidget("scrollbar", scrollbarwidgetclass, outer, wargs, num_args);

/* add scorlling procedure to callback list */ XtAddCallback (scrollbar, XtNscrollProc, Scrolled, NULL);

/* generate dialog widget and pulldown menus */ CreateformWithButton (outer);

/* realize all widgets */

xtRealizewidget (toplevel);

/* create a shared colormap */

c_colormap (canvasl);

/* convert data pixel value to color value for canvaso */ c_data (0);

/* create image and send image to display */

c_image (canvas, 0);

C_data (1);

C_image(canvas1, 1);

C_data (2) ;

c_image (canvas2, 2);

/* add mouse coursor position track event to event

* handler

*/

c_mouse_track (outer, canvas);

c_mouse_track(outer, canvasi);

c_mouse_track(outer, canvas2);

/ * callback procedure of redisplay */

XtAddCallback (canvas, XtNexpose, redisplay, NULL) ;

/* realize scrollbar widget */

XtRealizeWidget (scrollbar);

/* event loop, retrive event from event queue

* and invoke procedures

*

XtAppMainLoop (app_con) ;

\}

/* callback procedure of horizontal scrollbar */

static XtcallbackProc

Scrolled(w, closure, call_data)

widget $\mathrm{w}$; 


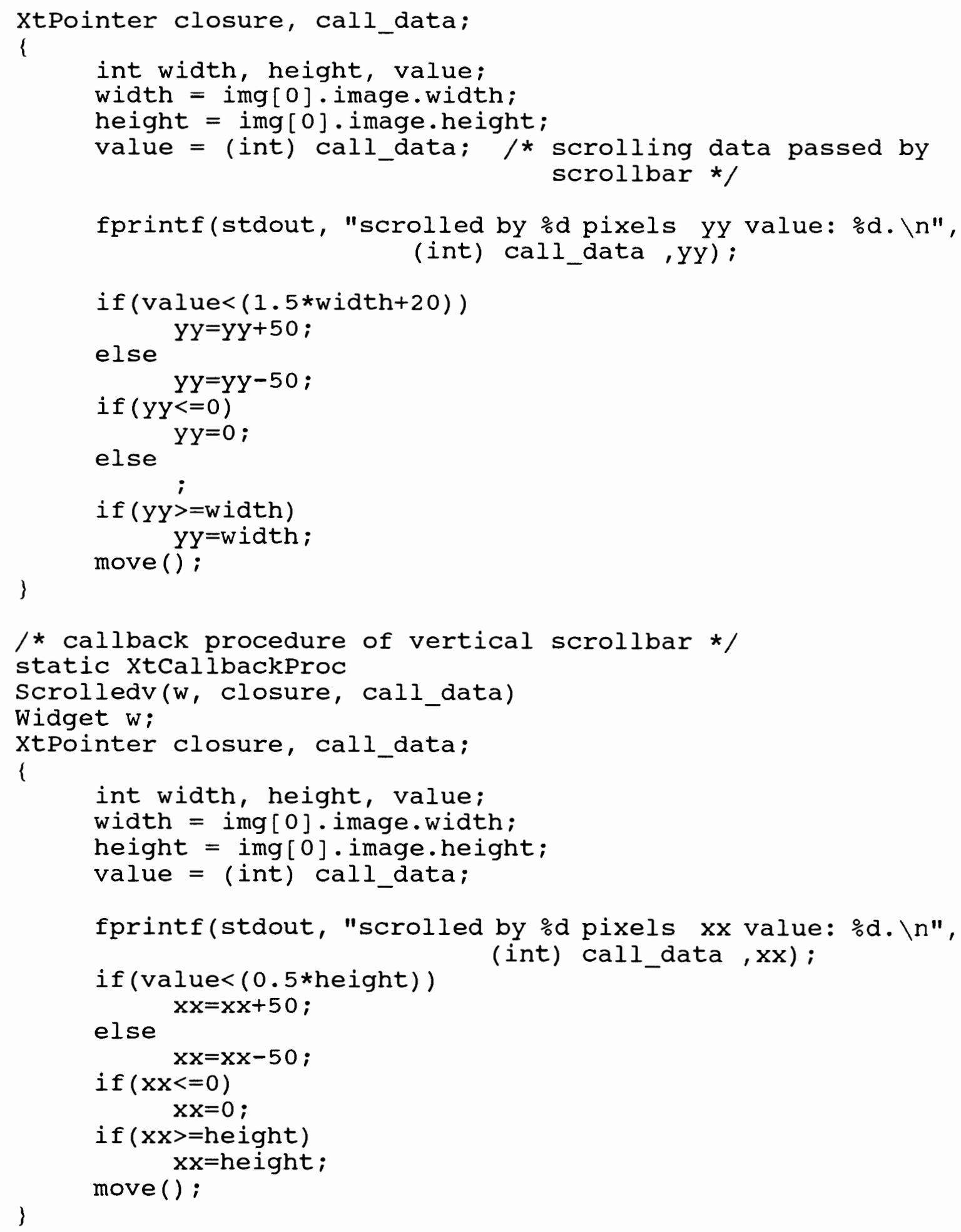




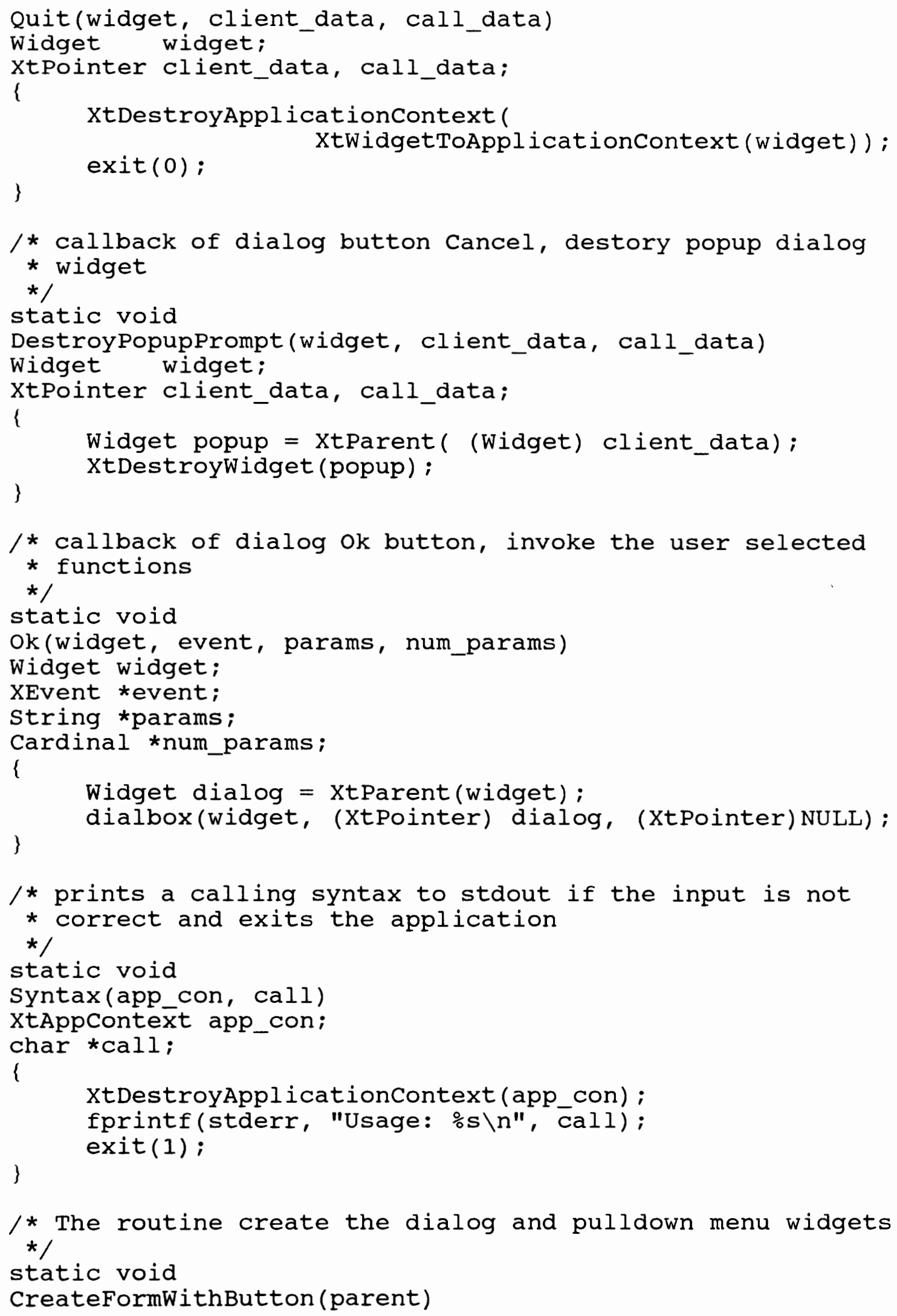


Widget parent;

Widget form, menu, entry;

Widget button, button1, button2, button3;

Widget button4, button5, button6, button7;

int $i$;

Arg wargs[3];

/* create a form type management widget */

form = XtcreateManagedWidget ("form", formWidgetclass, parent, NULL, ZERO);

/ create the dialog widget button */

button 1 = XtcreateManagedWidget ("Dialogbox", commandWidgetclass, form, NULL, ZERO);

/* add dialog procdure to callback list */

xtAddCallback(buttonl, xtNcallback, dial, NULL);

/* create a pulldown menu button named 'File' which is on

* the right of dialog button

*/

XtsetArg (wargs [0], XtNfromHoriz, button1);

button 2 = xtcreateManagedWidget ("File", menuButtonwidgetclass, form, wargs, ONE);

/* create the popup shell */

menu = XtcreatePopupshell ("menu", simpleMenuWidgetclass, button2, NULL, ZERO);

/* add submenu entry to the pulldown menu */

entry = XtcreateManagedWidget ("Quit", smeBSBObjectclass, menu, NULL, ZERO);

/* add callback procedure to callback list */

XtAddCallback (entry, XtNcallback, Quit,

(XtPointer) entry);

/* add submenu entry to the pulldown menu */

entry = XtCreateManagedWidget("Restore",

smeBSBObjectclass, menu, NULL, ZERO);

/* add callback procedure to callback list */

xtAddCallback (entry, XtNcallback, restore,

(xtPointer) entry) ;

/* create a second pulldown menu button, set it

* at horizontal right of the button 'File'

*/

xtsetArg (wargs [0], xtNfromHoriz, button2);

button 3 = xtcreateManagedWidget ("Edit", menuButtonWidgetclass, form, wargs, ONE) ; 
/* create the popup shell */

menu = XtcreatePopupshell ("menu", simpleMenuWidgetclass, button3, NULL, ZERO);

/* create the sub-button entries and add them to callback

* list

*/

entry $=$ xtcreateManagedwidget ("Zoom in", smeBSBObjectclass, menu, NULL, ZERO) ;

XtAddCallback (entry, XtNcallback, enlarge, (XtPointer) entry);

entry = XtcreateManagedwidget("Zoom out", smeBSBObjectclass, menu, NULL, ZERO);

XtAddCallback (entry, XtNcallback, reduce, (XtPointer) entry);

entry = XtCreateManagedWidget ("Rotat", smeBSBobjectclass, menu, NULL, ZERO);

XtAddCallback (entry, XtNcallback, rotation, (XtPointer) entry);

/* create a third pulldown menu button, set it

* at horizontal right of the button 'Edit'

*

xtsetArg(wargs[0], xtNfromHoriz, button3);

button 4 = XtCreateManagedWidget("Function", menuButtonwidgetclass, form, wargs, ONE) ;

menu = XtcreatePopupshell ("menu", simpleMenuWidgetclass, button4, NULL, ZERO);

entry = XtcreateManagedWidget("Media", smeBSBObjectclass, menu, NULL, ZERO);

XtAddCallback(entry, XtNcallback, media, (xtPointer) entry);

entry = XtCreateManagedWidget("Average", smeBSBObjectclass, menu, NULL, ZERO);

xtAddCallback (entry, xtNcallback, ave, (xtPointer) entry);

entry = xtcreateManagedWidget("Histogram", smeBsBobjectclass, menu, NULL, ZERO);

xtAddCallback (entry, XtNcallback, histo, (xtPointer) entry) ;

entry = xtcreateManagedWidget ("Sharp", smeBSBObjectclass, menu, NULL, ZERO);

xtAddCallback (entry, xtNcallback, sharp, (xtPointer) entry);

entry = XtcreateManagedWidget("Direct", smeBSBobjectclass, menu, NULL, ZERO);

xtAddCallback (entry, xtNcallback, direct, (xtPointer) entry);

entry = XtcreateManagedWidget("Diff", smeBsBobjectclass, menu, NULL, ZERO) ;

xtAddCallback (entry, xtNcallback, diff, (xtPointer) entry); 


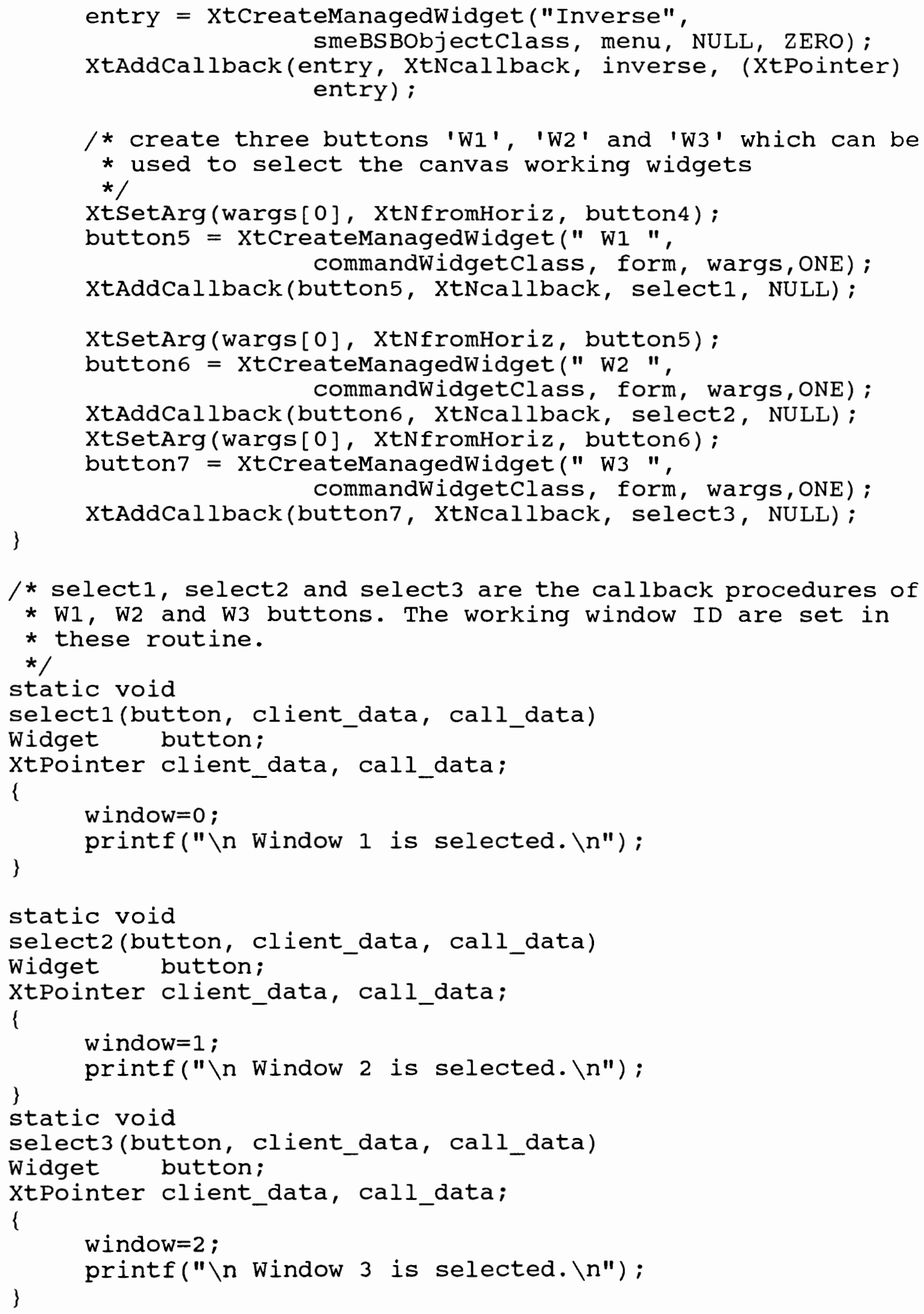




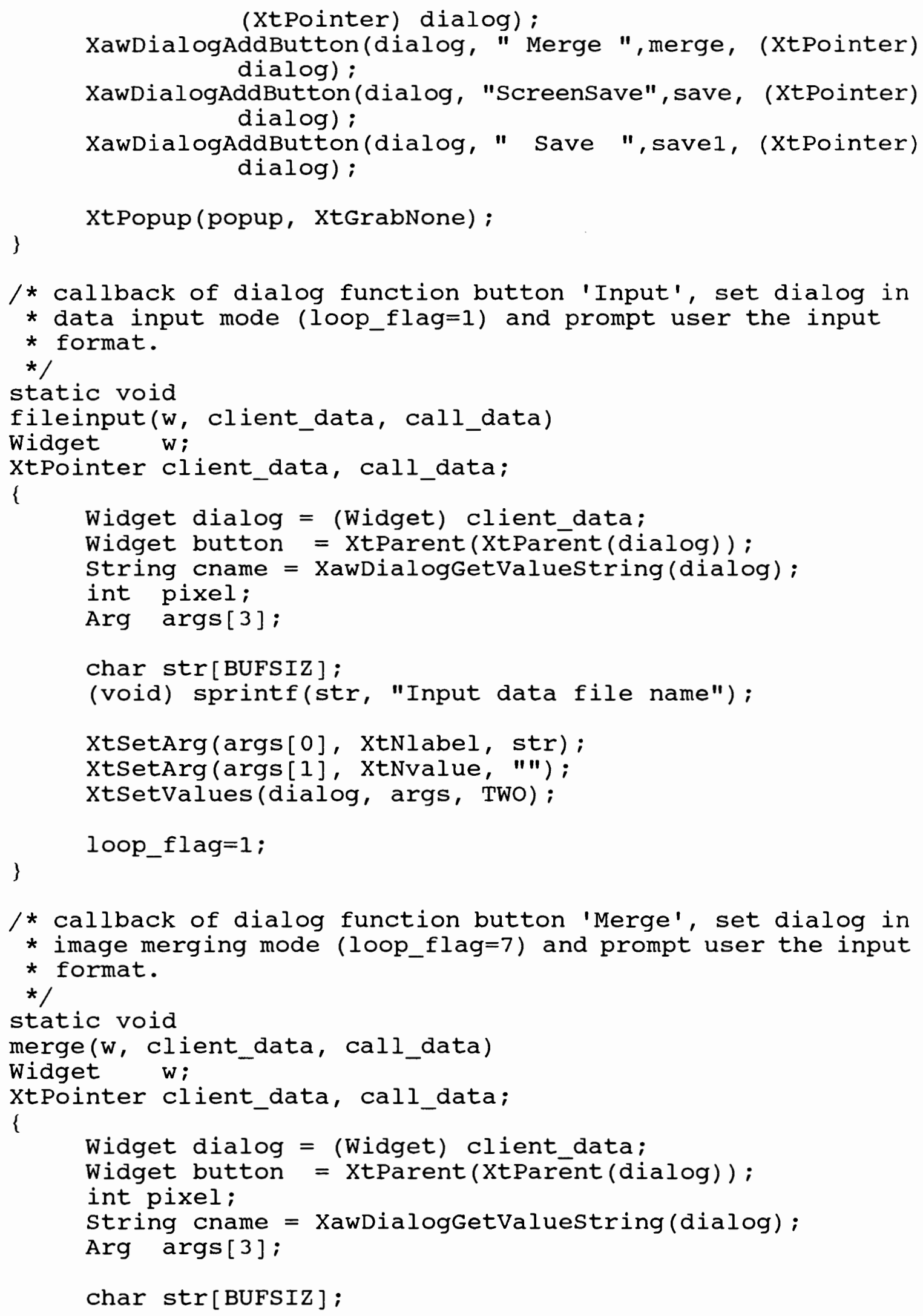


(void) sprintf(str, "Please 4 input file name or window name.");

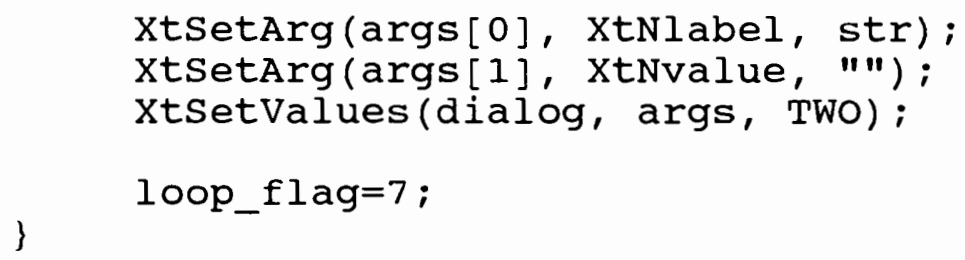




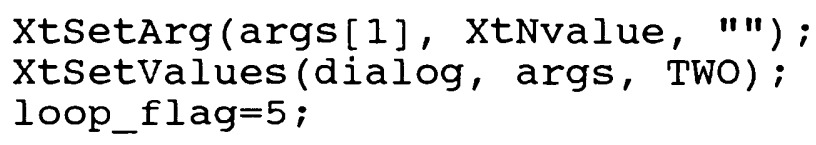




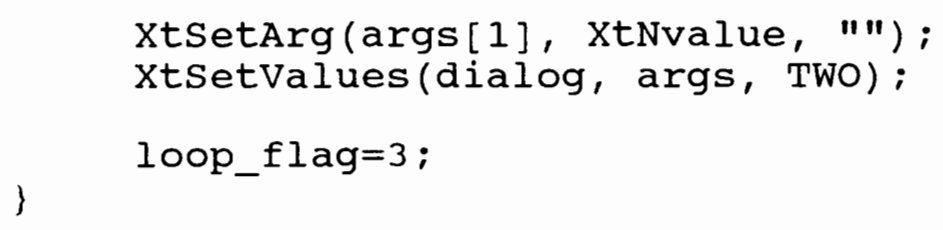




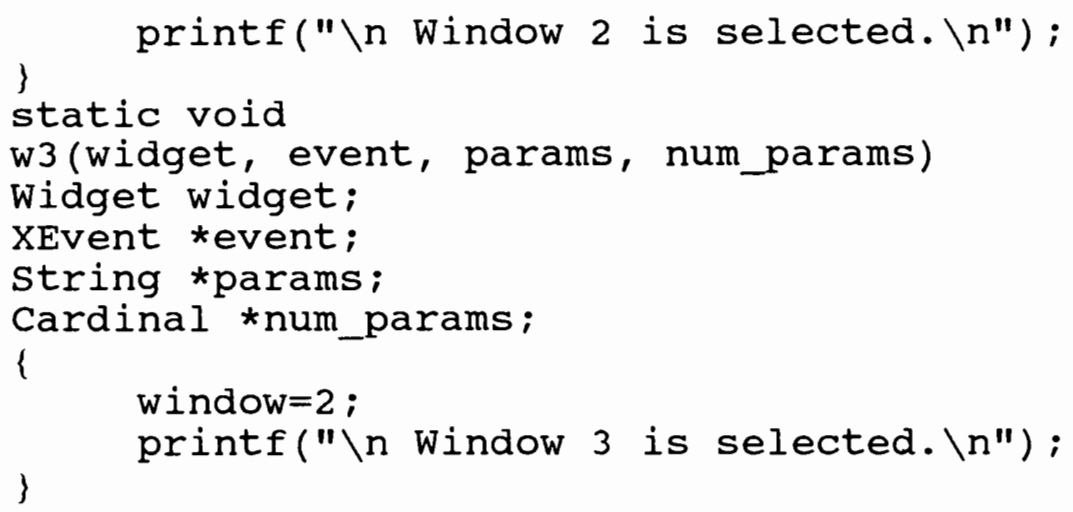




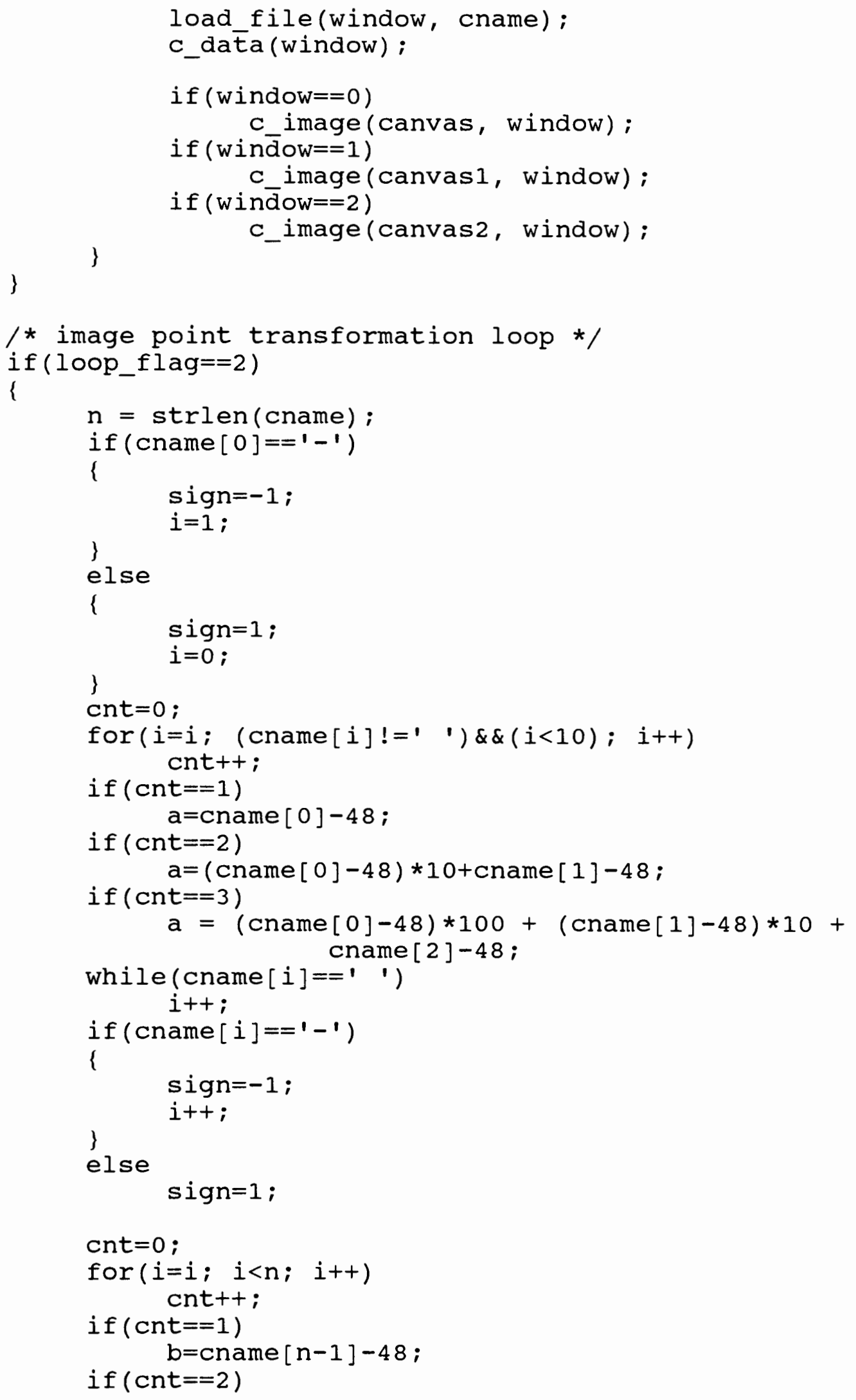




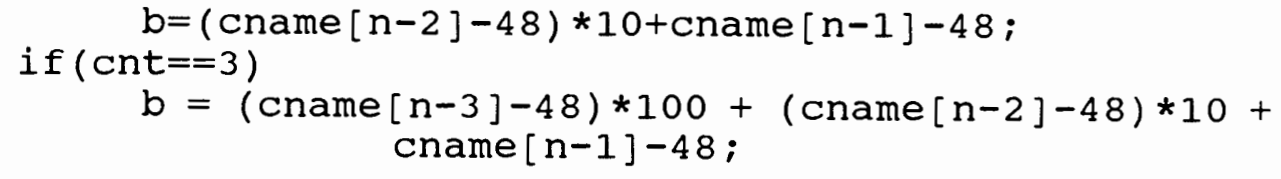

input $x=i m g$ [ window] . image. width;

inputy=img [window] . image. height;

if $((a==0) \& \&(b>0) \& \&($ sign= $=1))$

for $(x=0 ; x<=255 ; x++)$

for $\left(y=0 ; y<=255 ; y^{++}\right)$

\{

$\mathrm{n}=$ mydata $[$ window $]$. inimg $[\mathrm{x}][\mathrm{y}]$;

if $(n>b)$

mydata $[$ window $]$. inimg $[\mathrm{x}][\mathrm{y}]=0$;

\}

if $((a>0) \& \&(b==0) \& \&($ sign $==1))$

for $(x=0 ; x<=255 ; \mathrm{x}++)$

for $(y=0 ; y<=255 ; y++)$

\{

$\mathrm{n}=$ mydata $[$ window $]$. inimg $[\mathrm{x}][\mathrm{y}]$;

if $(n<a)$

mydata $[$ window $] \cdot$ inimg $[x][y]=0$;

\}

$\operatorname{if}((a>0) \& \&(b>0) \& \&(\operatorname{sign}==1))$

for $(x=0 ; x<$ input $x ; x++)$

for $\left(y=0 ; y<\right.$ inputy; $\left.y^{++}\right)$

\{

$\mathrm{n}=$ mydata $[$ window $]$. inimg $[\mathrm{x}][\mathrm{y}] ;$

if $((n<a)$ | $\mid(n>b))$

mydata $[$ window $]$. inimg $[x][y]=0$;

if ( $\operatorname{sign}==-1)$

for $(x=0 ; x<$ input $x ; x++)$

for $(y=0 ; y<$ inputy; $y++)$

\{

$\mathrm{n}=$ mydata [window]. inimg $[\mathrm{x}][\mathrm{y}] ;$

if $((n>a) \& \&(n<b))$

\}

mydata [window]. inimg $[\mathrm{x}][\mathrm{y}]=0$;

for $(x=0 ; x<$ input $x ; x++)$

for $\left(y=0 ; y<\right.$ inputy; $\left.y^{++}\right)$

$\mathrm{a}=\operatorname{mydata}[$ window $]$. inimg $[\mathrm{x}][\mathrm{y}] ;$

if $(a \div 2==0)$

else

*imgp=defs $[a / 2] \cdot p i x e l ;$

imgp++;

*imgp $=\operatorname{defs}[(a-1) / 2] \cdot p i x e]$;

if ( indow $==0$ )

c_image (canvas, 0$)$; 


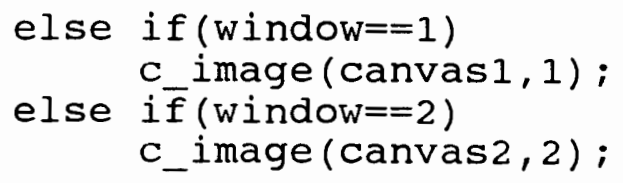


$($ cname $[x+1]-48) * 10+$ chame $[x+2]-48) ;$

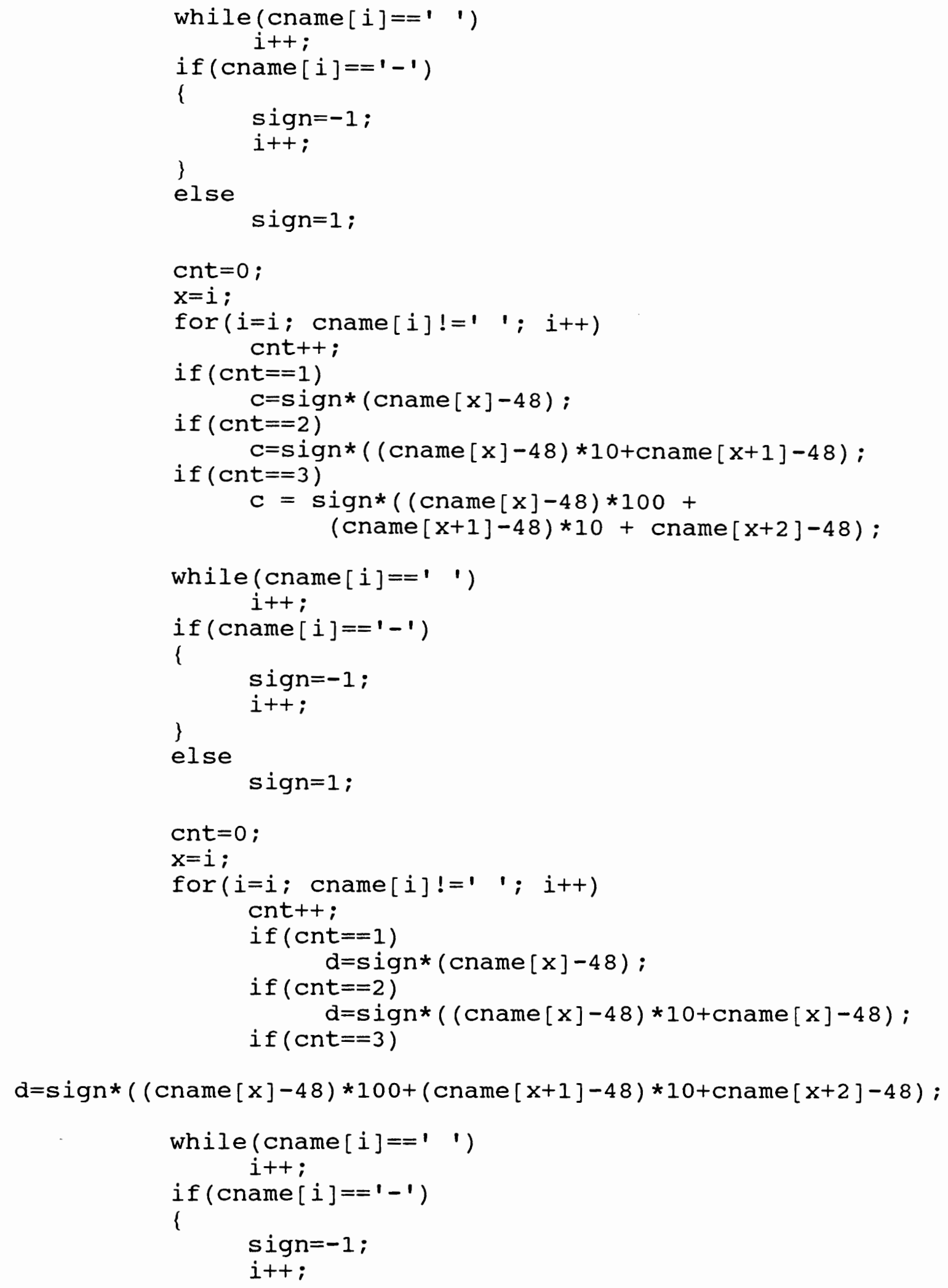




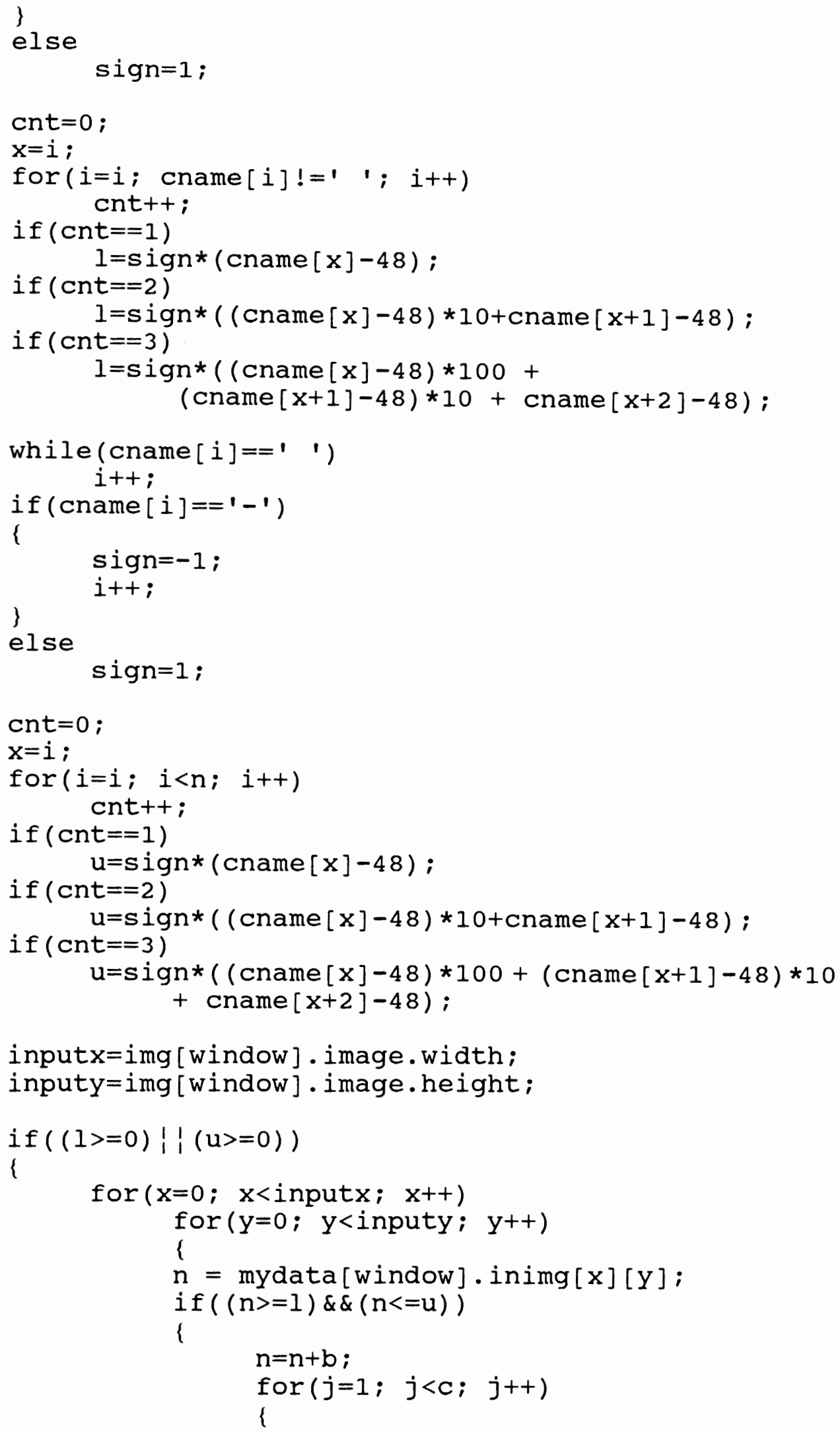




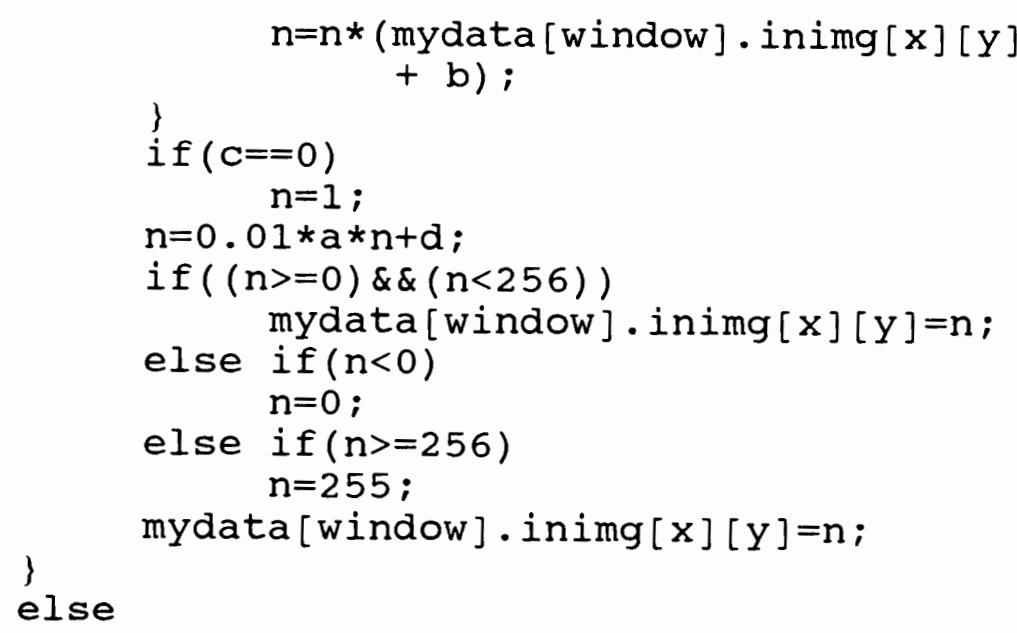
mydata $[$ window $]$. inimg $[x][y]=0$;

\}

else

\{

$\mathrm{n}=\operatorname{mydata}[$ window $]$. inimg $[\mathrm{x}][\mathrm{y}] ;$

if $((n<(-1 * 1))$ ! $(n>(-1 * u)))$

\{

$\mathrm{n}=\mathrm{n}+\mathrm{b}$;

for $(j=1 ; j<c ; j++)$

if $(\mathrm{C}==0)$

$n=n *($ mydata $[$ window $]$. inimg $[x][y]+b)$; $\mathrm{n}=1$

$\mathrm{n}=0.01 * \mathrm{a} * \mathrm{n}+\mathrm{d}$;

if $((n>=0) \& \&(n<256))$

else if $(n<0)$

$n=0$;

else if $(n>=256)$

$n=255$;

mydata [window]. inimg $[x][y]=n$;

\}

else

\}

mydata $[$ window $]$. inimg $[x][y]=0 ;$

for $(x=0 ; x<$ input $x ; x++)$

for $(y=0 ; y<$ inputy; $y++)$

\{

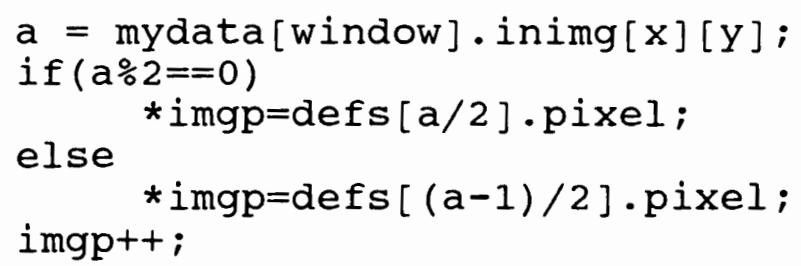




$$
\begin{aligned}
& \text { C_image (canvas, 0); } \\
& \text { else i } \bar{f} \text { ( } w \text { indow }==1 \text { ) } \\
& \text { C image (canvas } 1,1) \text {; } \\
& \text { else i } \bar{f} \text { (window==2) } \\
& \text { C_image (canvas } 2,2 \text { ); }
\end{aligned}
$$

\}

/* This routine capture the displayed image on the screen */

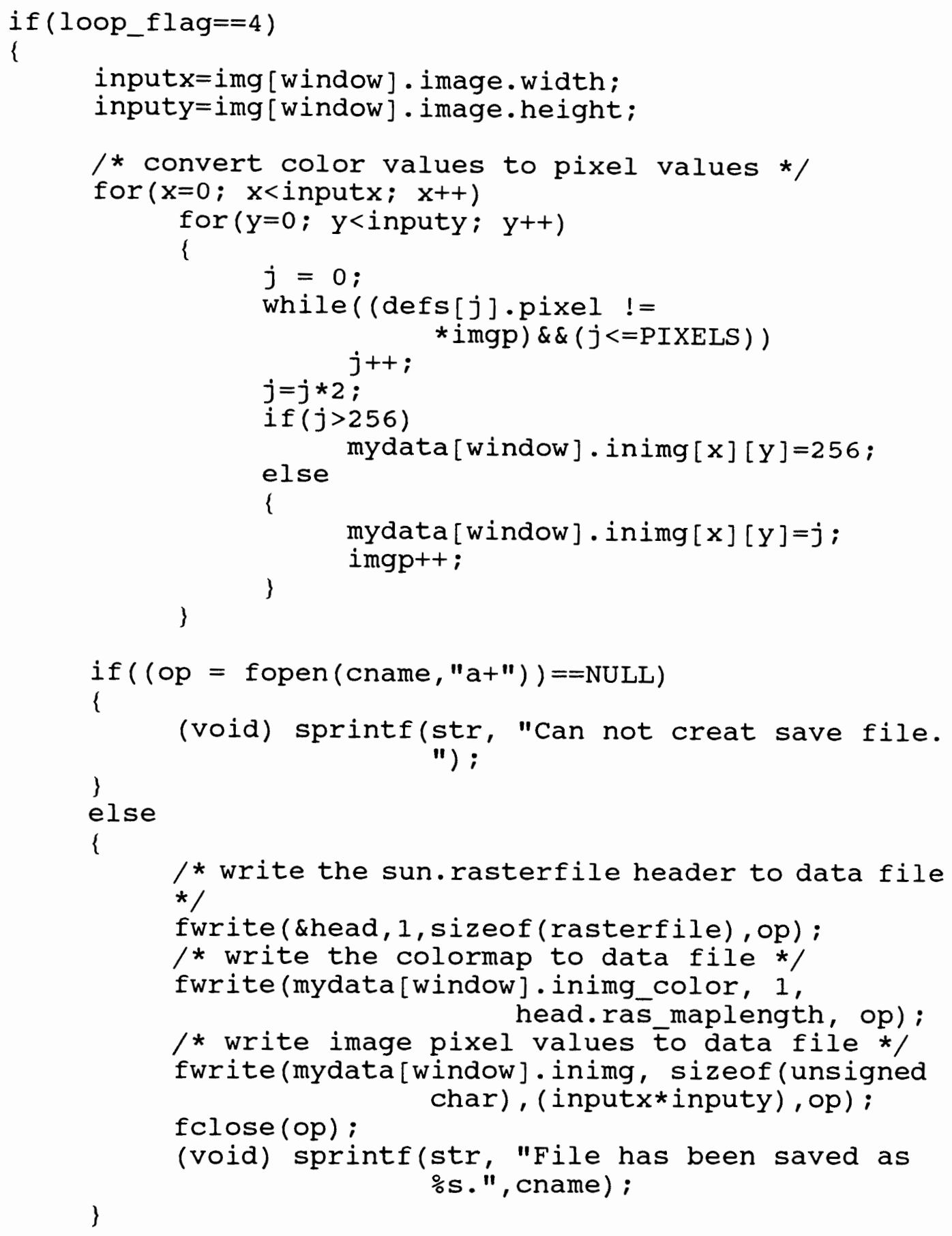




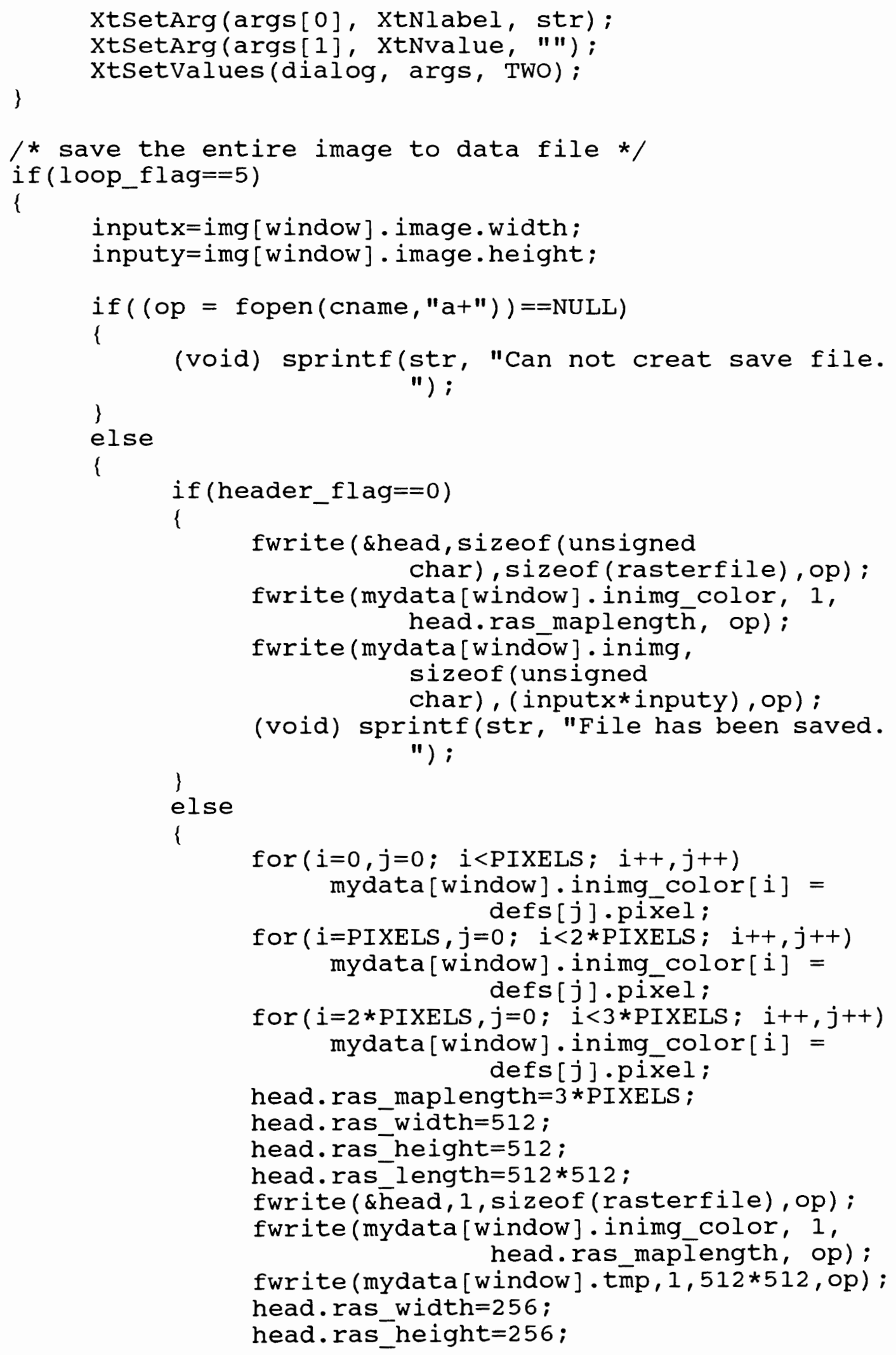




\section{head.ras_length $=256 * 256$; \\ \} \\ fclose (op) ; \\ \} \\ xtsetArg (args [0], XtNlabel, str); \\ xtsetArg(args [1], xtNvalue, " "); \\ \} \\ xtsetvalues(dialog, args, TWO);}

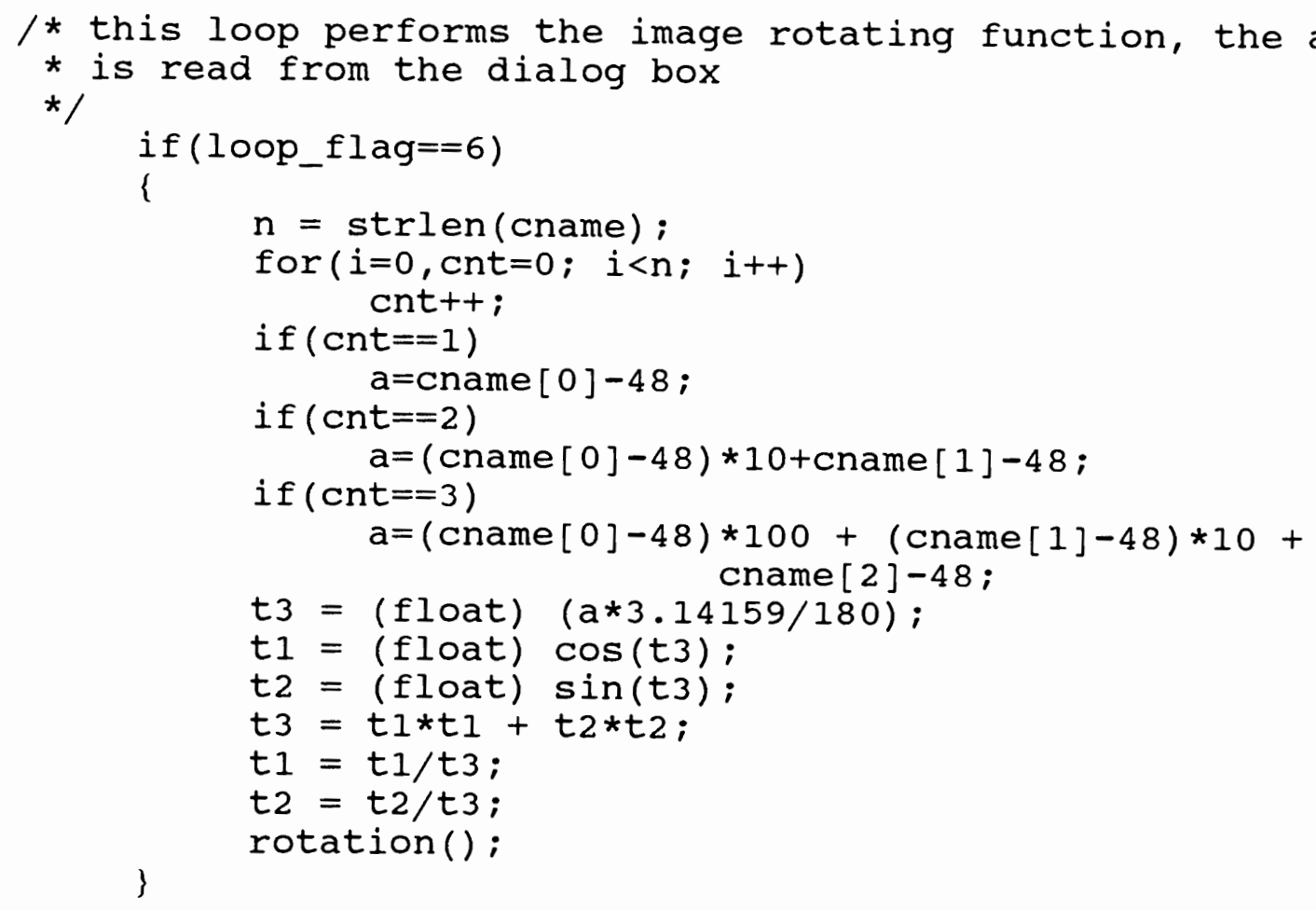

/* merge four image into one, the input can be either the

* window number or

* image file name

*/

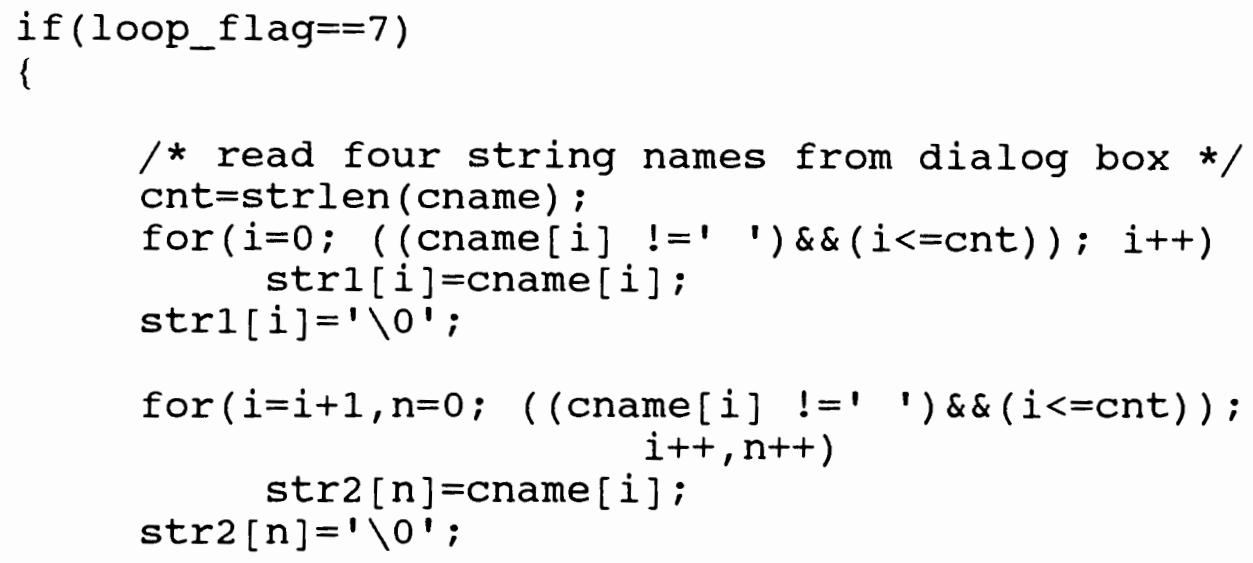




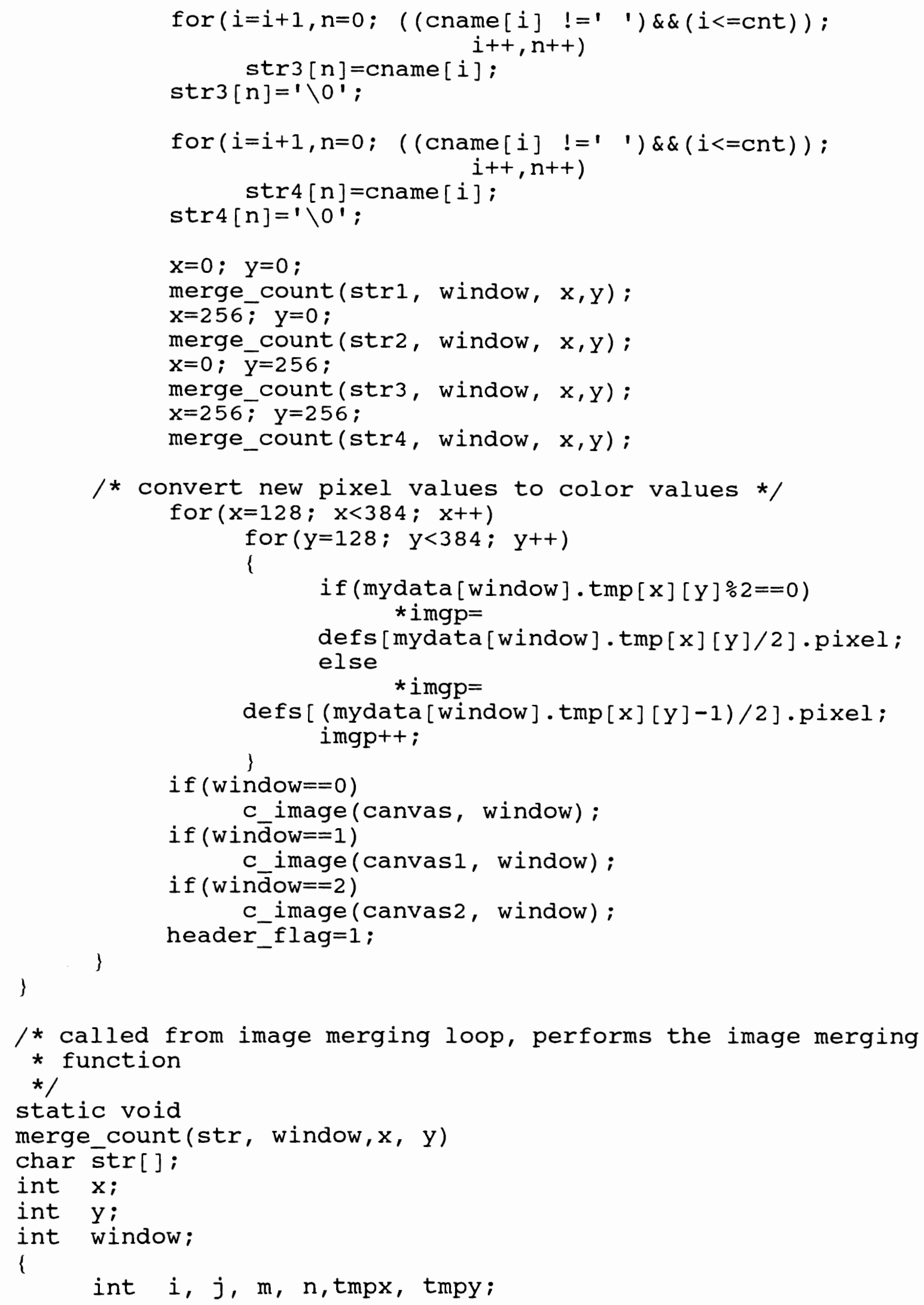




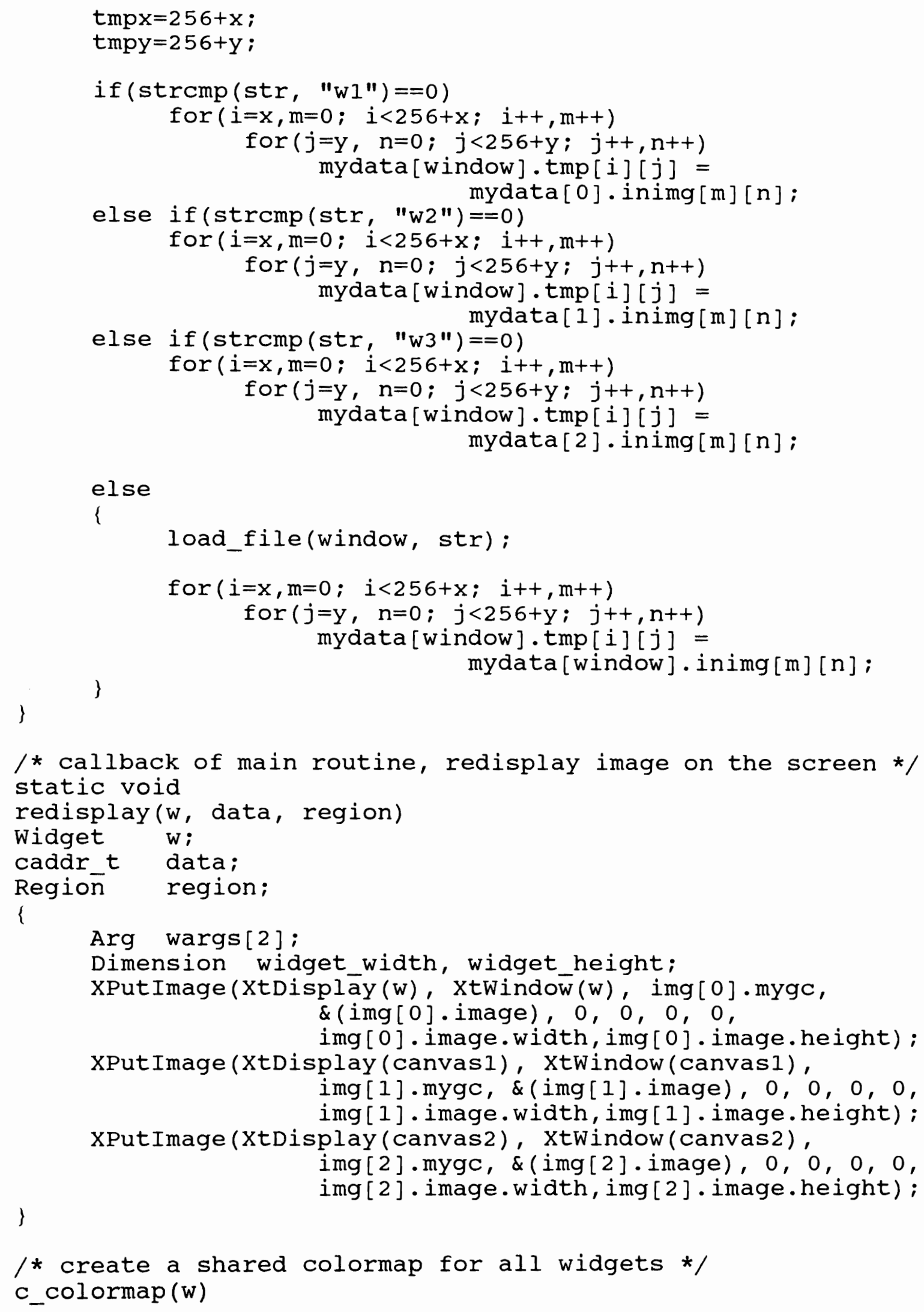

/* callback of main routine, redisplay image on the screen */ static void 


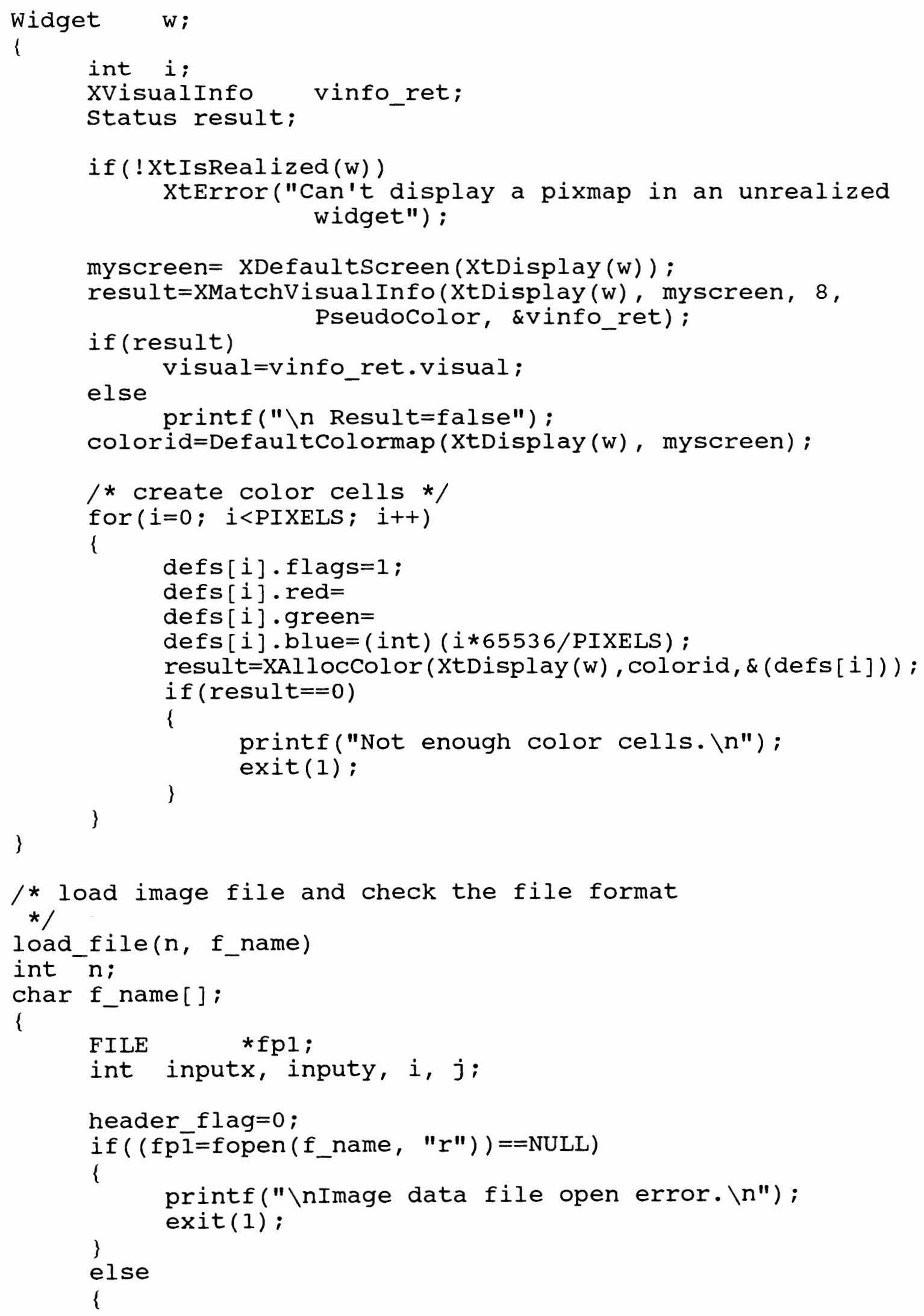




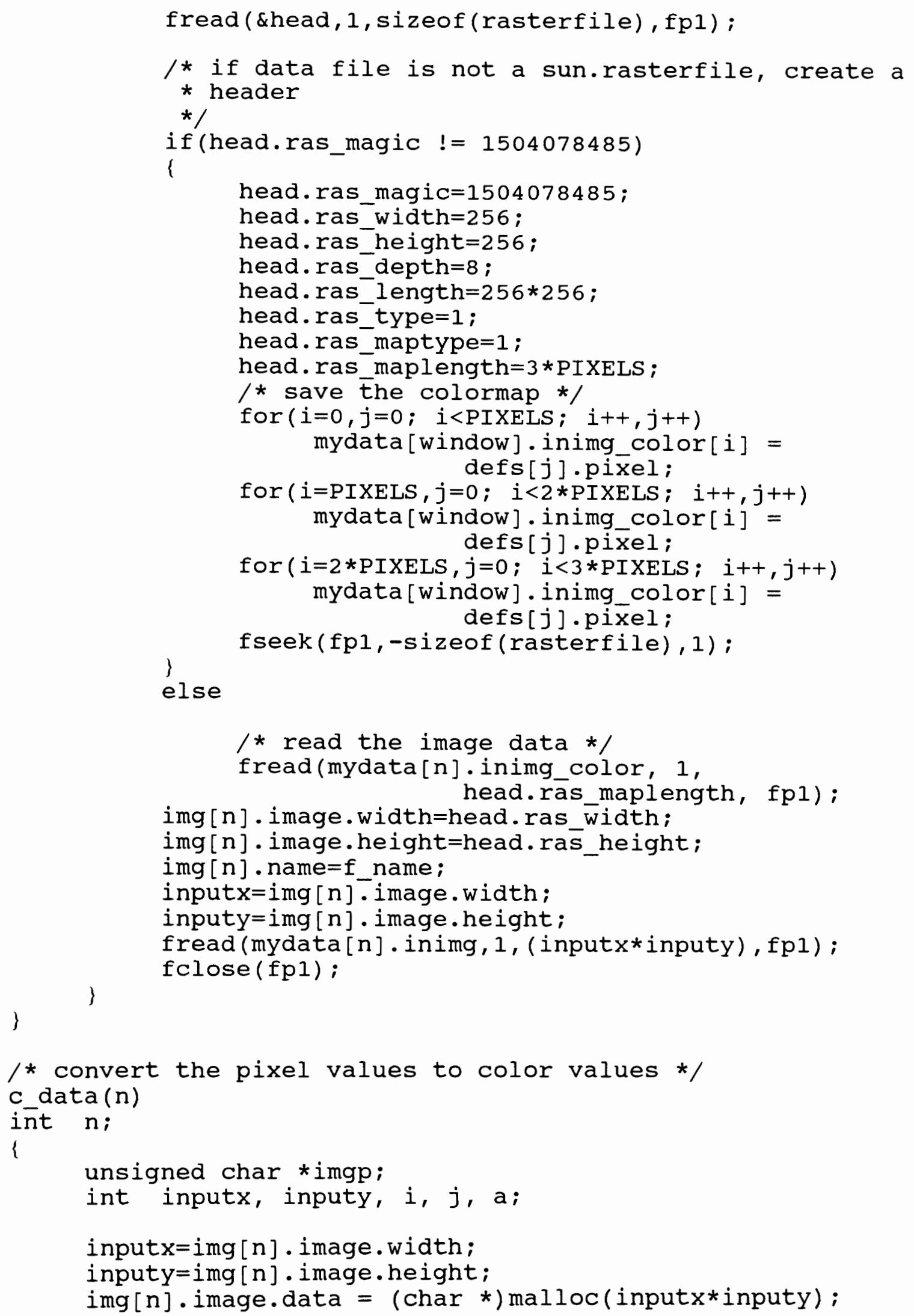




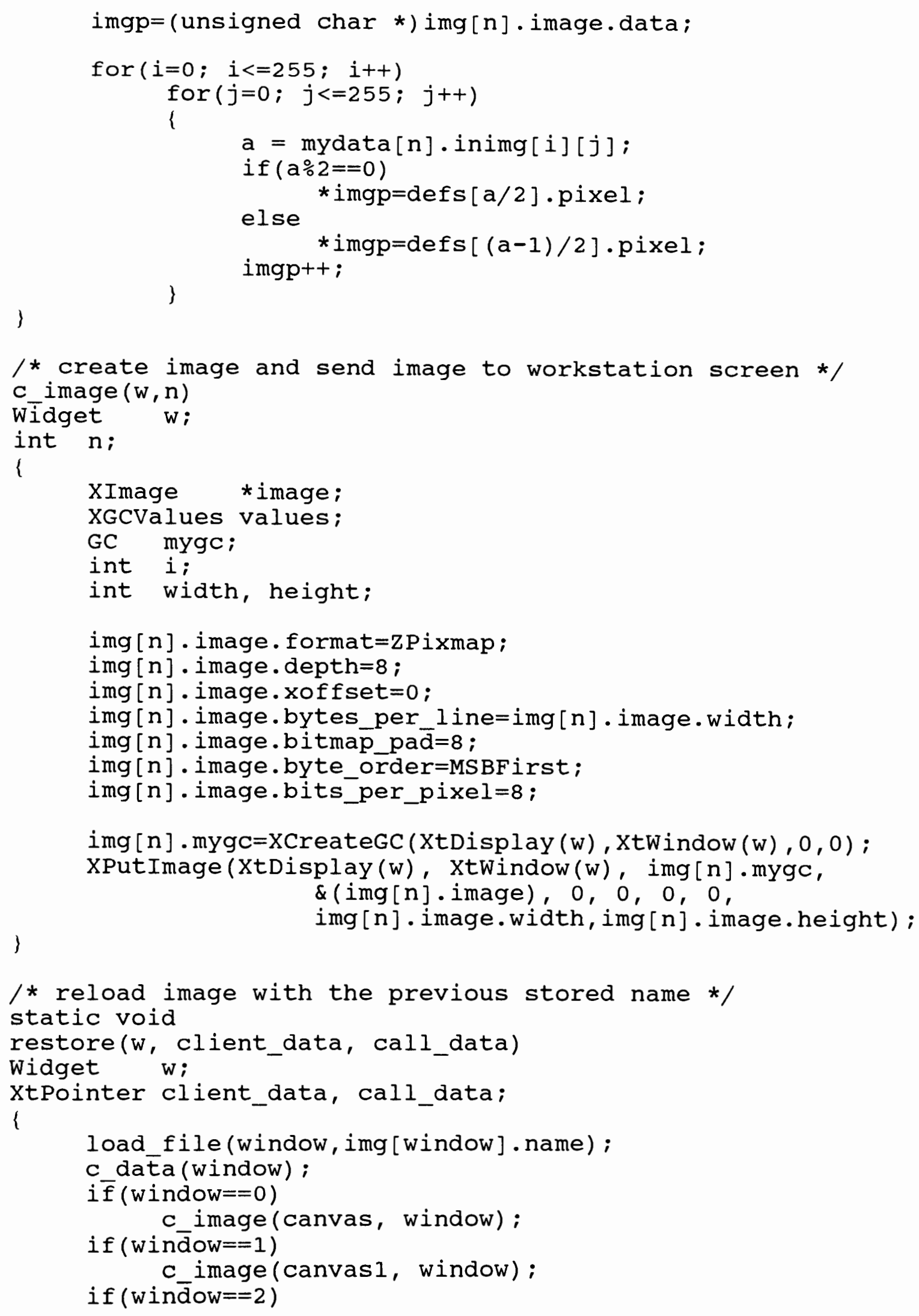


\}

c_image (canvas2, window);

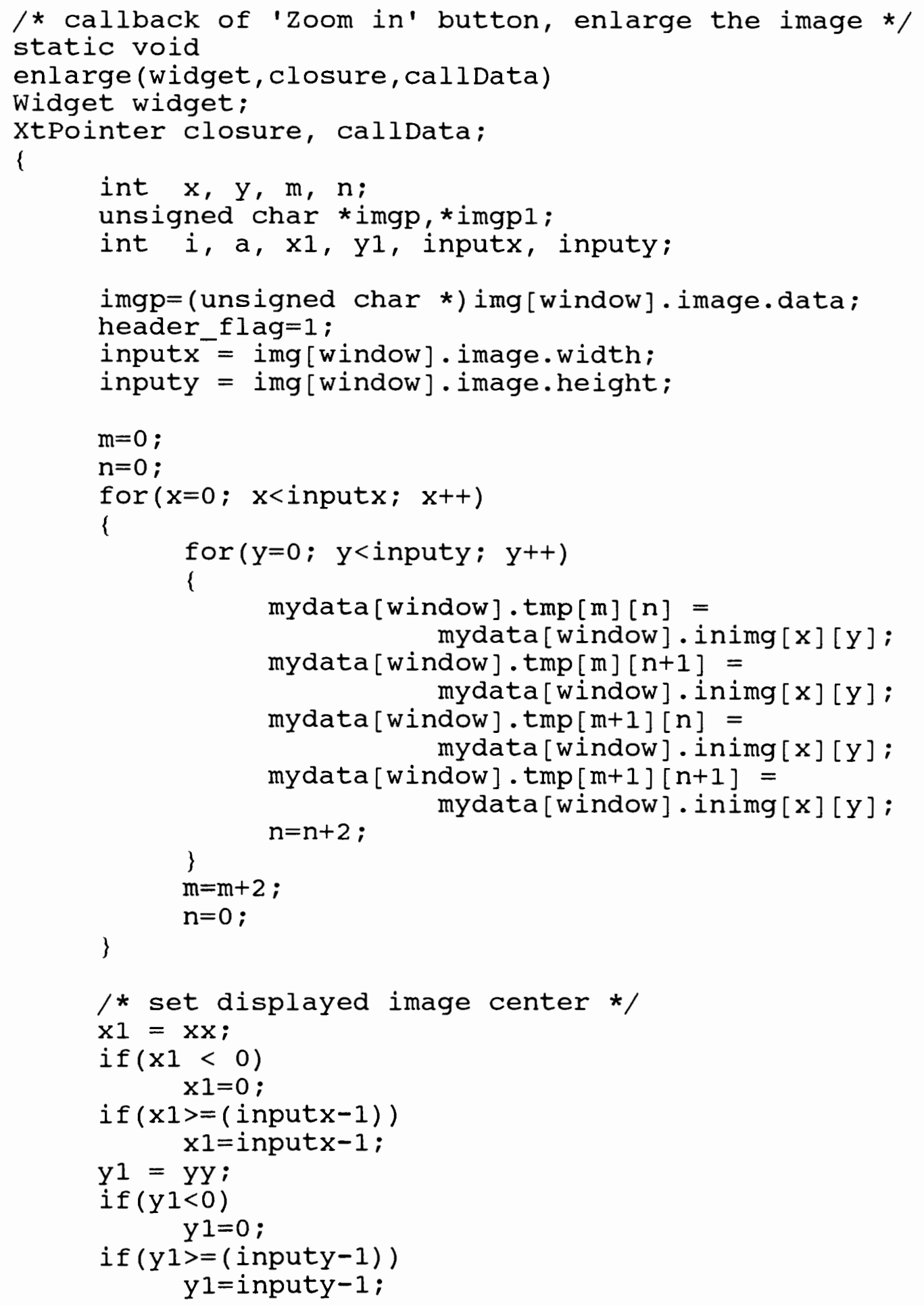

/* convert new image pixel values to color values */ for $(x=x 1 ; x<($ input $x+x 1) ; x++)$ 


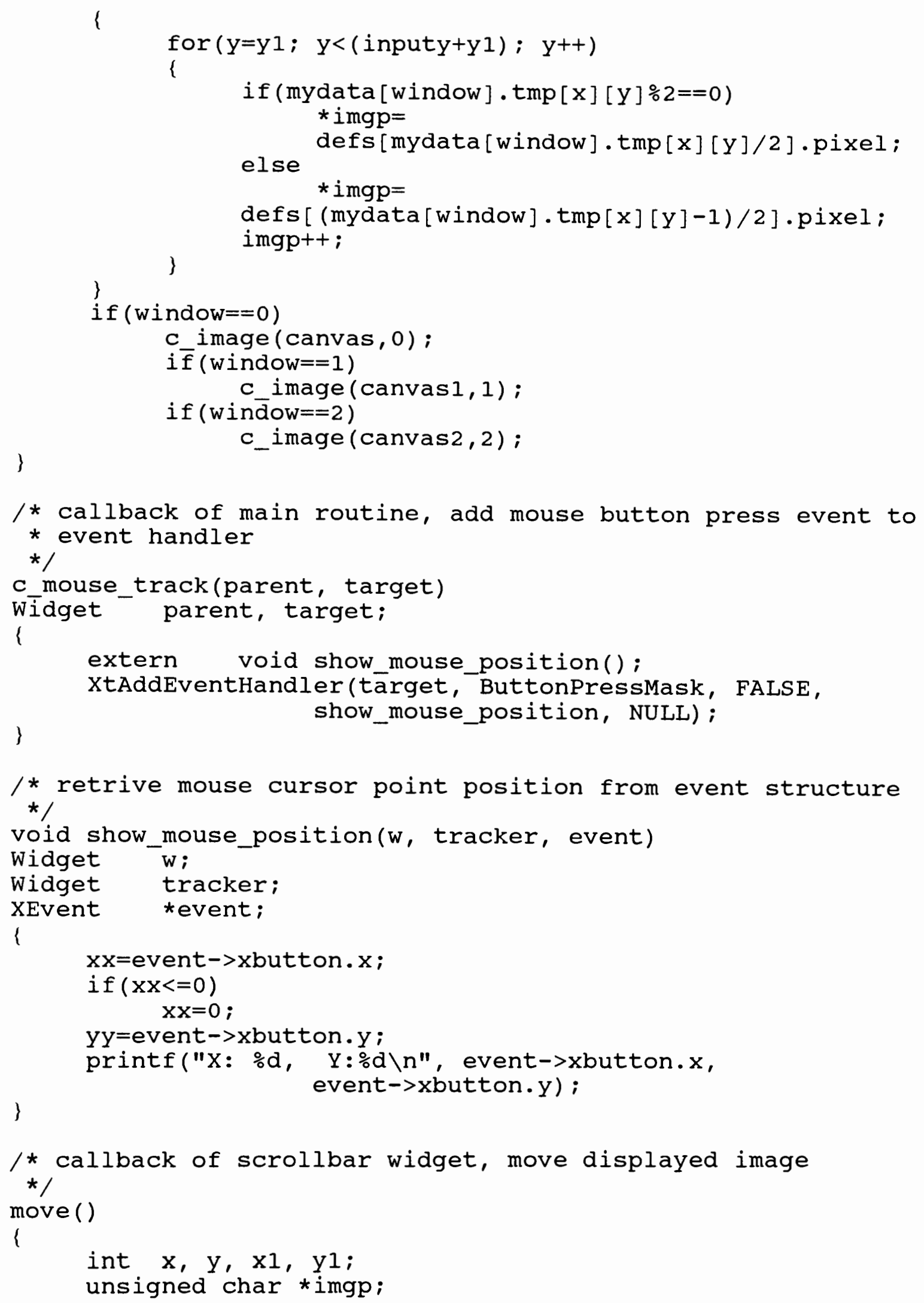


int a,inputx, inputy;

imgp=(unsigned char *) img[window]. image.data ;

inputx $=$ img [0]. image.width;

inputy $=$ img [0]. image.height;

/* set new displayed image center */

$\mathrm{x} 1=\mathrm{xx}$;

if $(x 1<0)$

$\times 1=0$;

if $(x 1>=($ input $x-1))$

$x 1=$ input $x-1$;

$\mathrm{Yl}=\mathrm{YY}$;

if $(y 1<0)$

$\mathrm{y} 1=0$;

if $(y 1>=($ input $y-1))$

$y 1=$ inputy-1;

for $(x=x 1 ; x<($ input $x+x 1) ; x++)$

\{

for $\left(y=y 1 ; y<(\right.$ inputy $\left.+y 1) ; y^{++}\right)$

\{
if ( (mydata [window] $\cdot \operatorname{tmp}[x][y] \div 2)==0)$
*imgp $=$
else
defs [mydata [window] . tmp [x] [y]/2] • pixel;

$\star \operatorname{imgp}=$

defs [(mydata [window] $\cdot \operatorname{tmp}[x][y]-1) / 2] \cdot p i x e l$; imgp+t;

\}

\}

if ( $w$ indow $==0$ )

c_image (canvas, 0 );

if ( $w$ ind $\bar{d} w==1$ )

C_image (canvas1,1);

if (window= $=2$ )

c_image (canvas 2,2 );

\}

/* media noise filter, with 3 by 3 sliding window *

static void

media(w, client_data, call_data)

Widget

w;

XtPointer client_data, call_data;

int $i, j$, inputx, inputy;

int $x, y, t m p$;

int $a$;

int data[20];

unsigned char

*imgp ; 


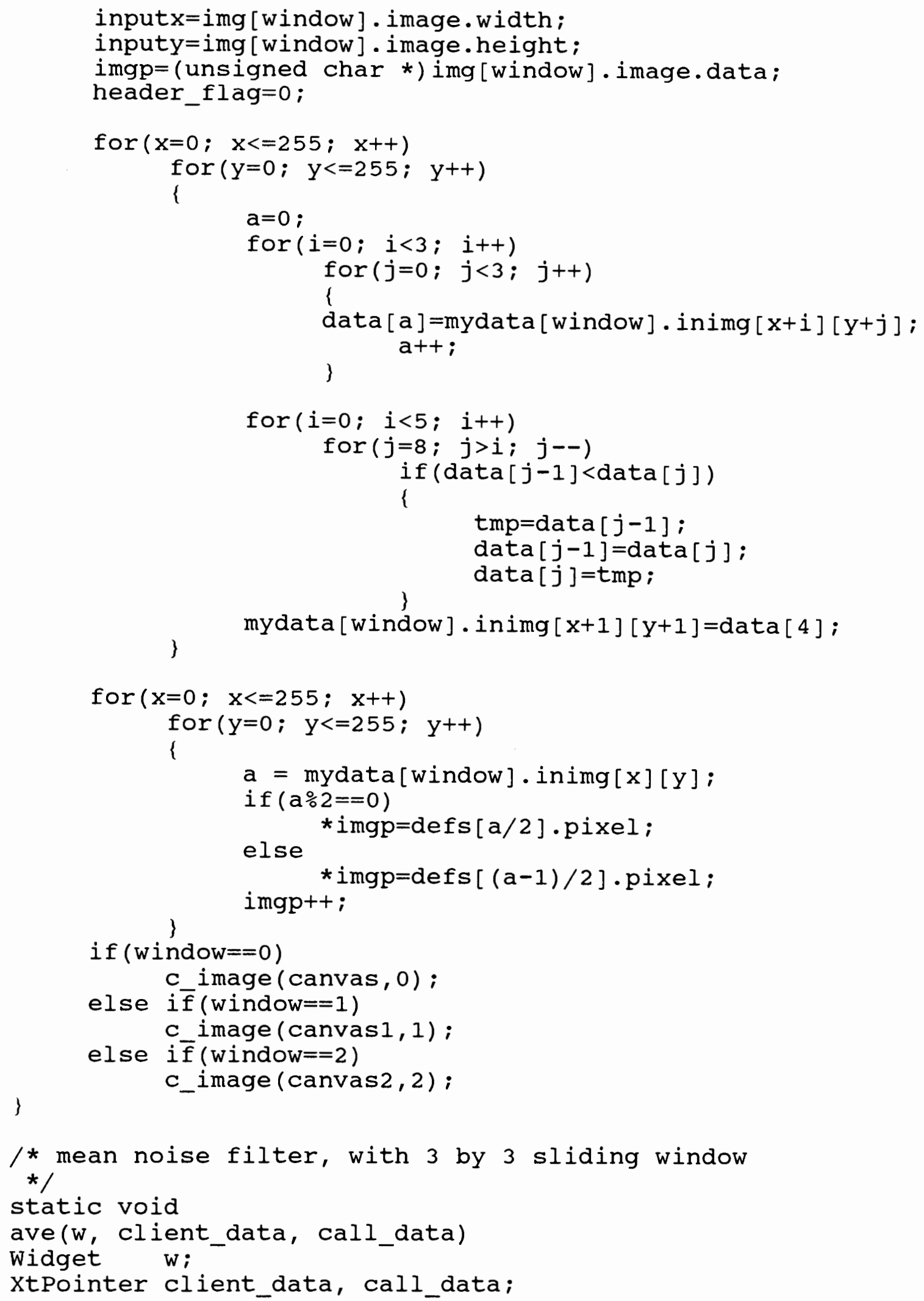




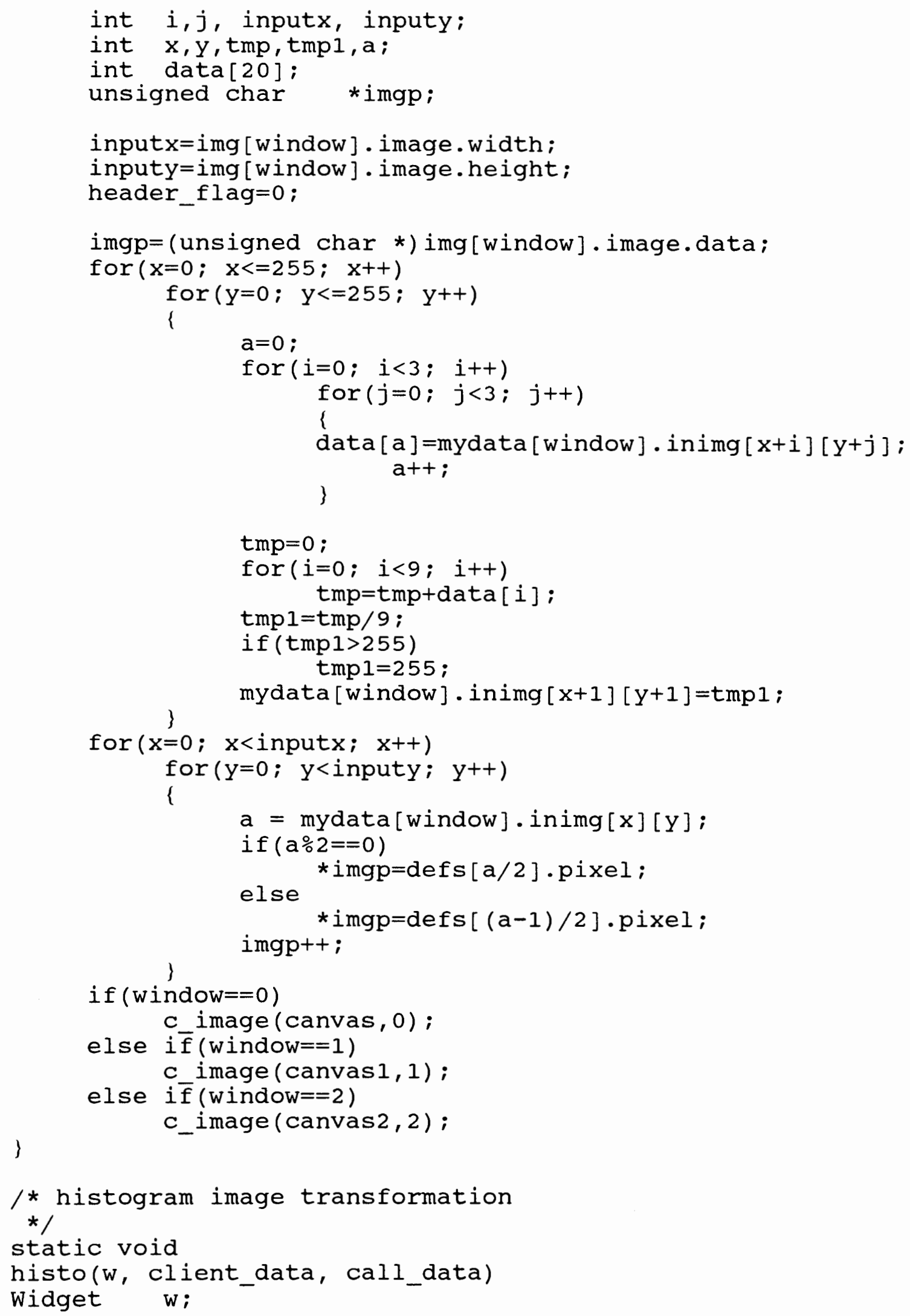


xtPointer client_data, call_data;

int $i, j$, inputx, inputy;

int $x, y, t m p, n, a$;

unsigned char *imgp;

double pr[256*256], sk[256*256];

header_flag $=0$;

input $x=i m g$ [window] . image. width;

inputy=img [window] . image . height ;

imgp=(unsigned char *) img[window]. image.data;

for $(i=0 ; i<256 ; i++)$

$\operatorname{pr}[i]=0$;

for $(x=0 ; x<$ inputx; $x++)$

for $\left(y=0 ; y<\right.$ inputy; $\left.y^{++}\right)$

i

$i=\operatorname{mydata}[$ window $]$. inimg $[x][y]$;

$\operatorname{pr}[\dot{i}]=\operatorname{pr}[i]+1$;

for $(i=0 ; i<256 ; i++)$

$\operatorname{pr}[i]=1.0 * \operatorname{pr}[i] /(256 * 256) ;$

for $(i=1 ; i<256 ; i++)$

$\operatorname{pr}[i]=\operatorname{pr}[i-1]+\operatorname{pr}[i]$;

for $(i=0 ; i<256 ; i++)$

\{

$j=0$;

for $(j=0 ; j<256 ; j++)$

\{

if $(((\operatorname{pr}[i]-(1.0 * j / 256))<0) \& \&(j<256))$

j-- ;

break;

for $(x=0 ; x<$ input $x ; x++)$

for $(y=0 ; y<i n p u t y ; y++)$

\{

if (mydata [window] . inimg $[x][y]==i$ )

\{

mydata [window] $\operatorname{inimg}[x][y]=j$;

\} tmp=n;

\}

for $(x=0 ; x<$ input $x ; x++)$

for $(y=0 ; y<$ inputy; $y++)$

\{

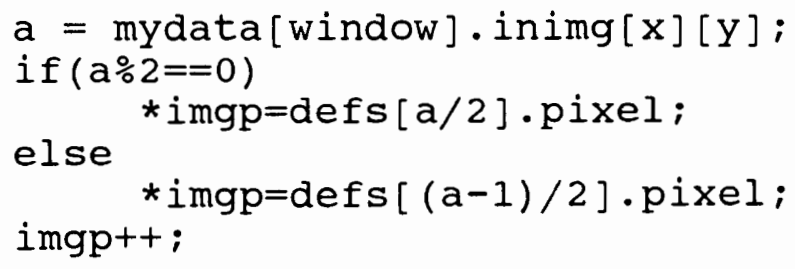




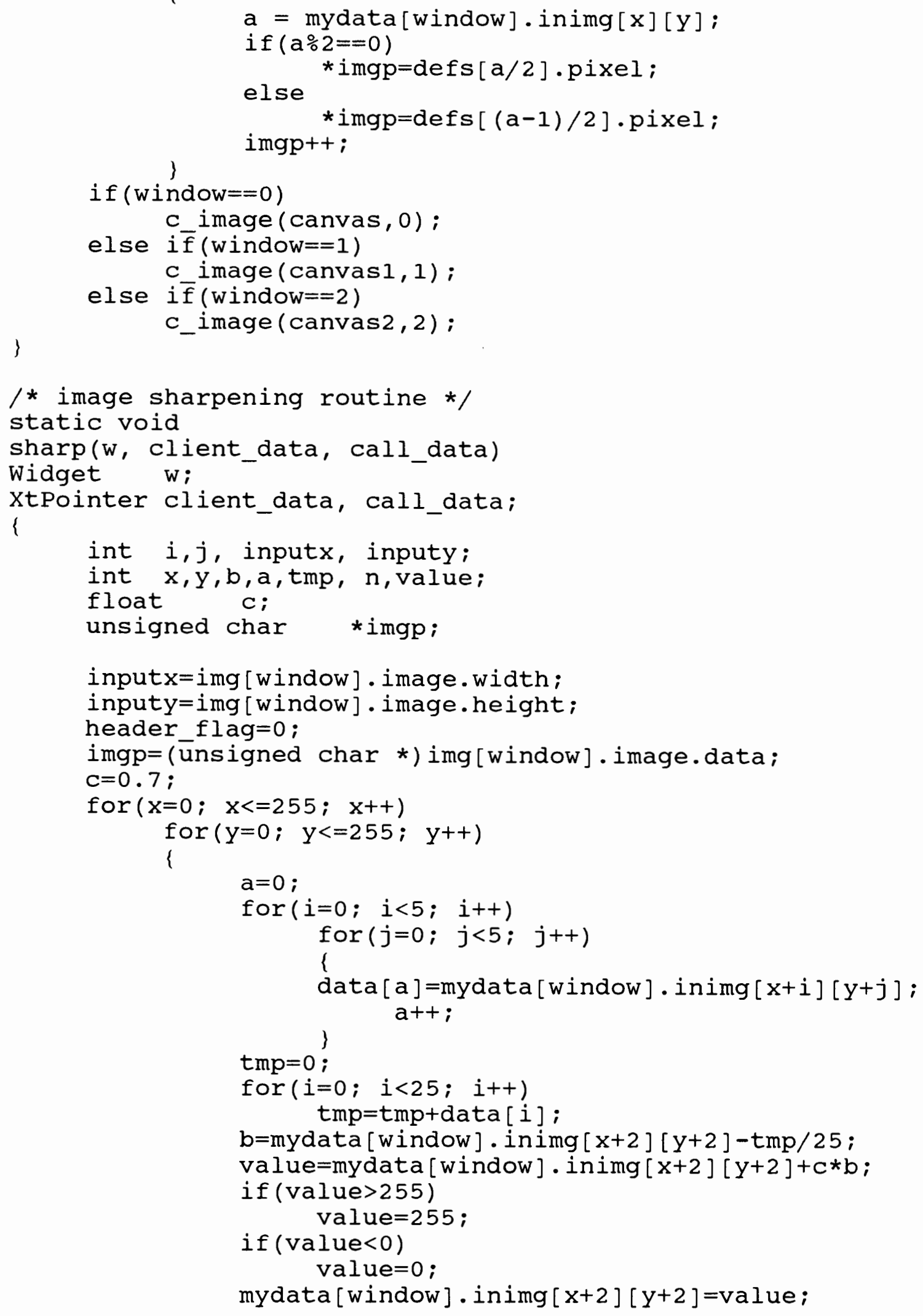




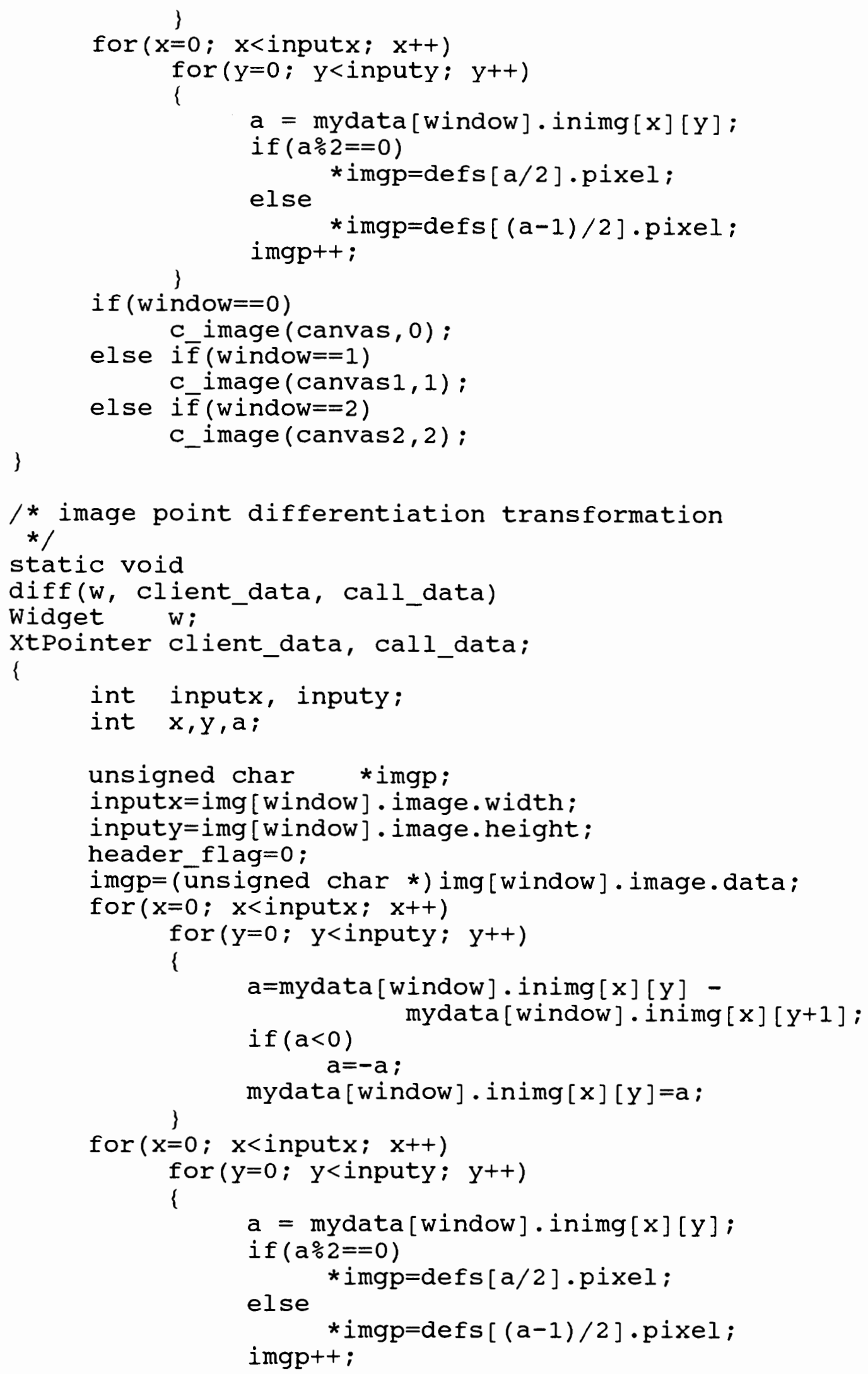




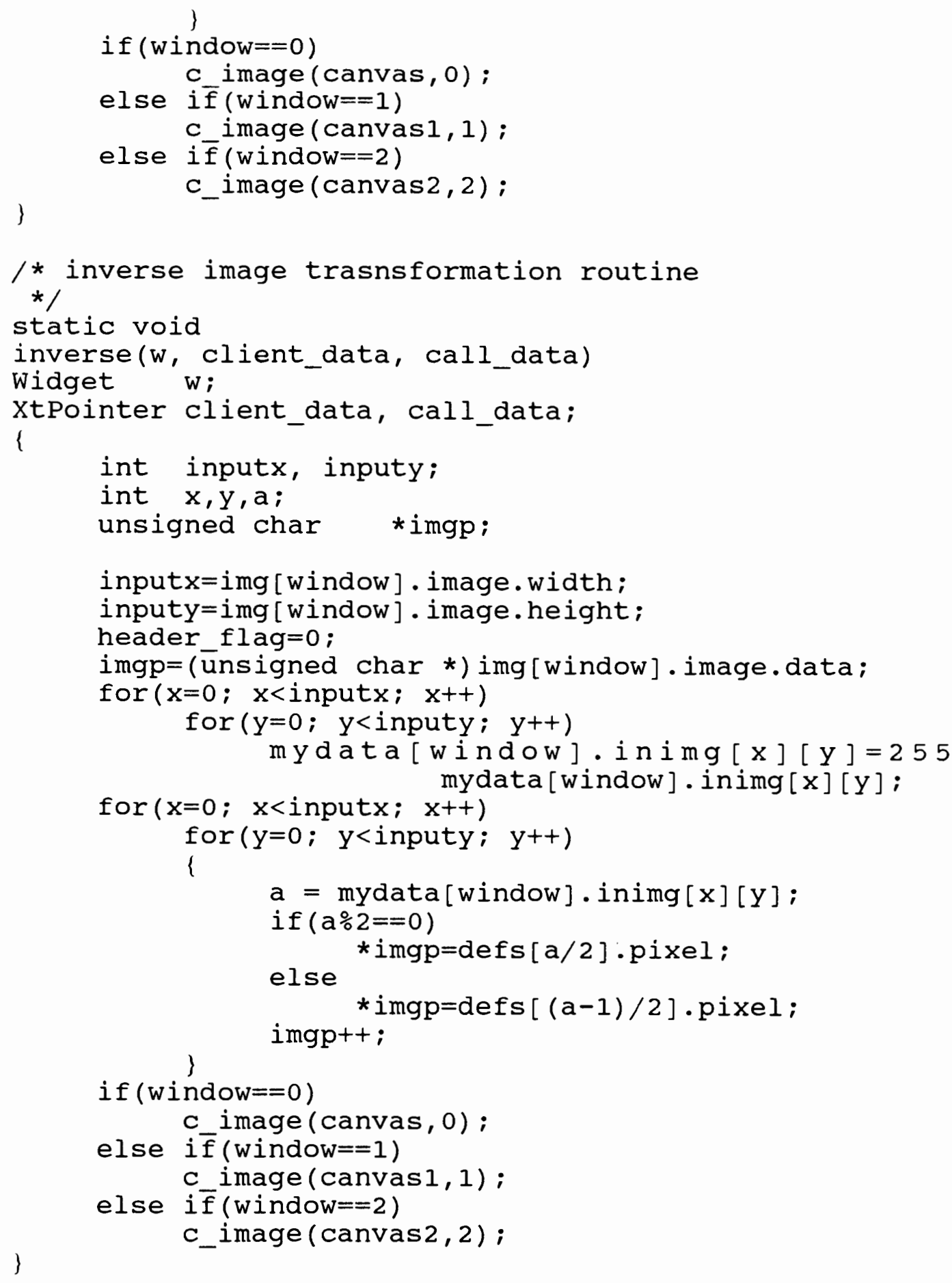

/* rotate image around a given center and angle, the angle 
int inputx, inputy;

int $x, y, b, a, m, n$;

unsigned char tmp[256][256];

unsigned char *imgp;

input $x=i m g$ [window] . image. width;

inputy =img [window] . image. height ;

header_flag $=0$;

imgp $=(\bar{u}$ nsigned char *) img[window]. image.data ;

$\mathrm{a}=\mathrm{xx}$;

$\mathrm{b}=\mathrm{YY}$;

for $(x=0 ; x<=255 ; x++)$

for $(y=0 ; y<=255 ; y++)$

$m=($ int $)(b-(b-x) * t 1+(y-a) * t 2) ;$

$\mathrm{n}=($ int $)(\mathrm{a}+(\mathrm{y}-\mathrm{a}) * t 1+(b-x) * t 2)$;

if $((m>255)$ i $(m<0)$ i $(n>255)$ | $(n<0))$

\{

$\mathrm{m}=0$;

$\mathrm{n}=0$;

mydata [window] inimg $[\mathrm{m}][\mathrm{n}]=255$;

(

$\operatorname{tmp}[x][y]=\operatorname{mydata}[$ window $]$. inimg $[m][n]$;

for $(x=0 ; x<=255 ; x++)$

\{

for $(y=0 ; y<=255 ; y++)$

\{

mydata $[$ window $]$. inimg $[x][y]=\operatorname{tmp}[x][y]$;

if $(\operatorname{tmp}[x][y] \% 2==0)$

else

*imgp=defs $[\operatorname{tmp}[x][y] / 2] \cdot p i x e l ;$

*imgp $=\operatorname{defs}[(\operatorname{tmp}[x][y]-1) / 2] \cdot \operatorname{pixel}$;

imgpt+ ;

\}

\}

if ( $w$ indow $==0$ )

C_image (canvas, 0);

else $i \bar{f}$ (window $==1$ )

C_image (canvasl, 1);

else i $\bar{f}$ ( $w$ indow $==2$ )

C_image (canvas2, 2);

\}

/* callback of 'Zoom out' button, reduce enlarged image *I

static void

reduce ()

int $i, j$, inputx, inputy;

int $x, y, a$;

unsigned char *imgp; 


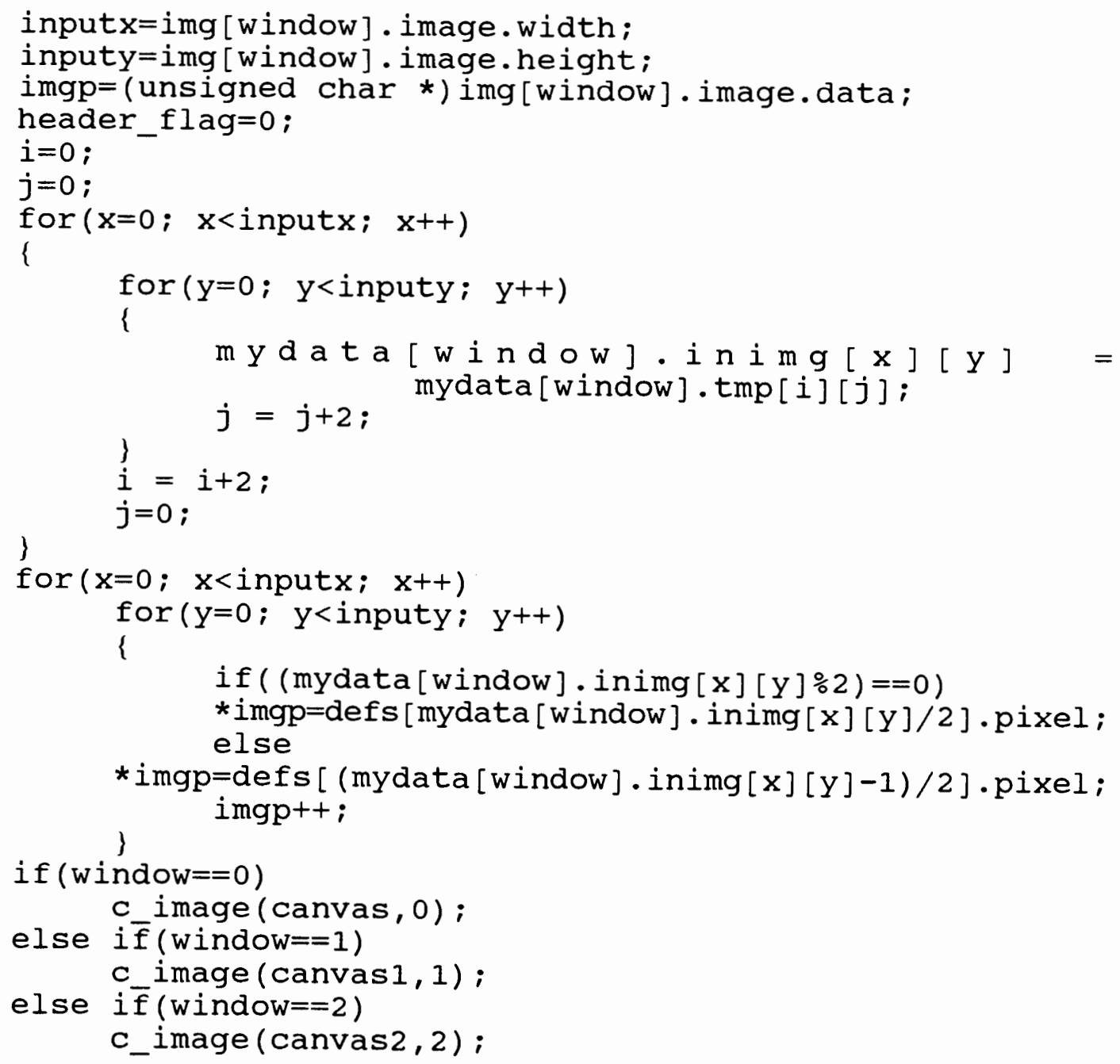


APPENDIX B

RESULTS 


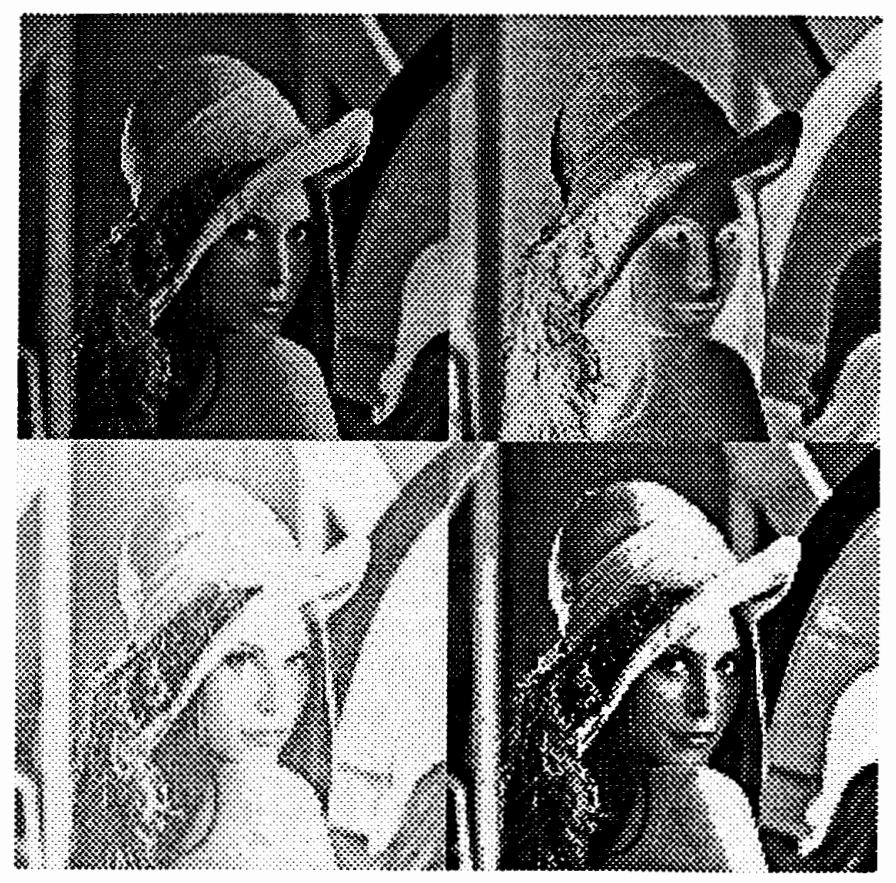

Figure 15. Output result: Upper-left, original image. Upper-right, inverted image. Lower-left, organelle image. Lower-right, histogram equalized image.

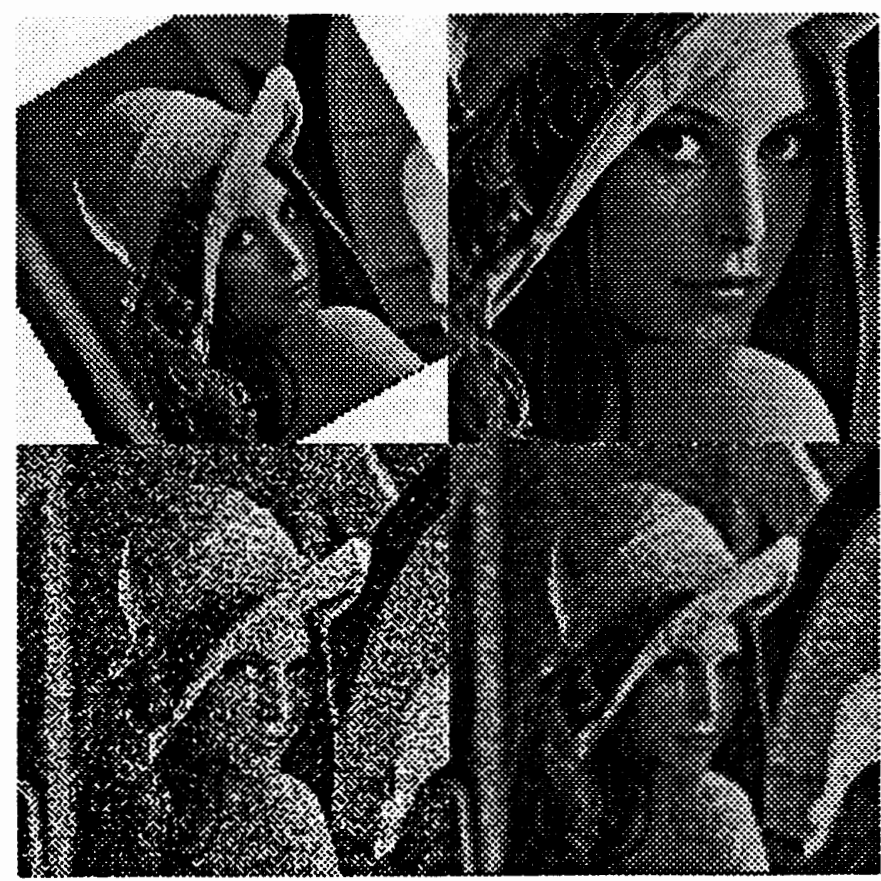

Figure 16. Output result: Upper-left, 30 degree rotated image around center. Upper-right, zoomed image. Lower-left, image with noise. Lower-right, result of $3 \times 3$ directional mean filtering and image sharping. 